\title{
MODEL THEORY AND ROKHLIN DIMENSION FOR COMPACT QUANTUM GROUP ACTIONS
}

\author{
EUSEBIO GARDELLA, MEHRDAD KALANTAR, AND MARTINO LUPINI
}

\begin{abstract}
We show that, for a given compact or discrete quantum group $G$, the class of actions of $G$ on $\mathrm{C}^{*}$-algebras is first-order axiomatizable in the logic for metric structures. As an application, we extend the notion of Rokhlin property for $G$-C*-algebra, introduced by Barlak, Szabó, and Voigt in the case when $G$ is second countable and coexact, to an arbitrary compact quantum group $G$. All the the preservations and rigidity results for Rokhlin actions of second countable coexact compact quantum groups obtained by Barlak, Szabó, and Voigt are shown to hold in this general context. As a further application, we extend the notion of equivariant order zero dimension for equivariant *-homomorphisms, introduced in the classical setting by the first and third authors, to actions of compact quantum groups. This allows us to define the Rokhlin dimension of an action of a compact quantum group on a $\mathrm{C}^{*}$-algebra, recovering the Rokhlin property as Rokhlin dimension zero. We conclude by establishing a preservation result for finite nuclear dimension and finite decomposition rank when passing to fixed point algebras and crossed products by compact quantum group actions with finite Rokhlin dimension.
\end{abstract}

\section{INTRODUCTION}

The Rokhlin property is a freeness condition for actions of groups on $\mathrm{C}^{*}$-algebras. It has been intensively studied in recent years due to, among other things, the strong implications it has on the structural properties of fixed point algebras and crossed products. While generalizations to noncompact groups, such as $\mathbb{Z}[32]$, or $\mathbb{R}$ [27], have been considered, the most common setting where the Rokhlin property has been studied is the one of finite or, more generally, compact groups; see [17, 29]. In this case, it has recently been shown implicitly [1], and explicitly in [22], that the Rokhlin property is of model-theoretic nature. Namely it corresponds to the *-homomorphism defining the action being positively existential in the sense of first order logic for metric structures.

Building on this work, the notion of Rokhlin property and the above-mentioned preservation results have been generalized to the more general setting of actions of coexact compact quantum groups on $\mathrm{C}^{*}$-algebras in [2]. This work has significantly expanded the scope of the preservation results for Rokhlin actions, paving the way of finding several new examples of classifiable $\mathrm{C}^{*}$-algebras arising from compact quantum group actions. Furthermore, approach from [2] has also contributed to a simplification and better understanding of the Rokhlin property, even in the classical setting.

While very fruitful, the Rokhlin property is also quite restrictive. In order to circumvent this problem, the notion of Rokhlin dimension has been recently introduced in the setting of actions of finite groups [28], compact groups [19], or $\mathbb{R}[27]$. In this setting, the Rokhlin property corresponds to having Rokhlin dimension equal to zero. Furthermore, unlike Rokhlin actions, actions with finite Rohlin dimension are prevalent, even in the case of $\mathrm{C}^{*}$-algebras that do not admit any Rokhlin action, such as the Jiang-Su algebra.

The model-theoretic description of the Rokhlin property has been generalized to Rokhlin dimension in [22], in terms of the notion, introduced therein, of equivariant order zero dimension for an equivariant *-homomorphism. Such a notion subsumes the notion of positively existential equivariant *-homomorphism, which corresponds to

Date: March 6, 2018.

2000 Mathematics Subject Classification. Primary 20G42, 46L55, 54H05; Secondary 03E15, 37A55.

Key words and phrases. C*-algebra, compact quantum group, quantum group action, Rokhlin property, Rokhlin dimension, logic for metric structures, axiomatizable class, positive existential embedding.

This work was initiated during a visit of E.G. and M.L. to the Mathematisches Forschungsinstitut Oberwolfach in August 2016, supported by an Oberwolfach Leibnitz Fellowship of M.L. Parts of this work were carried out during a visit of E.G. and M.K. to the California Institute of Technology in January 2017, and during a visit of E.G. and M.L. to the Centre de Recerca Matemàtica in March 2017 in occasion of the Intensive Research Programme on Operator Algebras. The authors gratefully acknowledge the hospitality and the financial support of all these institutions. E.G. was partially funded by SFB 878 Groups, Geometry and Actions, and by a postdoctoral fellowship from the Humboldt Foundation. M.K. was partially supported by the NSF Grant DMS1700259. M.L. was partially supported by the NSF Grant DMS-1600186. This work is part of the project supported by the grant H2020-MSCA-RISE-2015-691246-QUANTUM DYNAMICS.. 
having equivariant order zero dimension equal to zero. This new perspective has been used in [22] to recover and extend various preservation results for fixed point algebras and crossed products of actions with finite Rokhlin dimension.

The goal of this paper is to show how these notions and results naturally extend to the more general setting of actions of compact quantum groups. To this purpose, we begin by showing that, for a fixed discrete or compact quantum group $G$, the class of actions of $G$ on $C^{*}$-algebras $\left(G\right.$-C $C^{*}$-algebras) is first-order axiomatizable in a suitable language in the logic for metric structures. This provides a notion of positively existential $G$-equivariant *-homomorphism between $G$-C ${ }^{*}$-algebras, as well as a notion of ultraproducts and reduced products for $G$-C ${ }^{*}$ algebras consistent with the one considered in [2]. This perspective is then used to show how to remove all coexactness and separability assumptions in the preservation and rigidity results from [2].

We then consider the natural generalization to the notion of equivariant order zero dimension for equivariant *-homomorphisms, and define Rokhlin dimension for compact quantum group actions in terms of such a notion. We use the perspective of order zero dimension to establish many relevant facts about actions with finite Rokhlin dimension, some of which are new even in the well studied case of finite group actions. We conclude by showing how the preservation results for nuclear dimension and decomposition rank under fixed point algebras and crossed product from $[19,22,28]$ admit natural extensions to this setting.

In order to make the present paper accessible to readers that are not necessarily familiar with quantum groups, we recall all the definitions and results that we use. The interested reader can find more information in the monographs [6, 47] and the surveys [34, 35, 39]. Both compact and discrete quantum groups are subsumed by the more general class of locally compact quantum groups as defined and studied in [36-38, 48]. While this allows one to give a unified treatment, it is also technically more demanding. In order to make the paper more accessible, and since all our results regard quantum groups that either compact or discrete, we will present all the notions that we consider in these special cases. The interested reader is referred to [36-38, 48] as well as the monograph [47] for more information on locally compact quantum groups.

For convenience of the readers unfamiliar with first order logic for metric structures, we include an appendix containing the notions and results from the logic for metric structures that are used in the present paper. We will work in the framework of logic for metric structures with domains of quantification as considered in [15]. A good introduction to this topic is offered by the monograph [3]. A systematic study of $\mathrm{C}^{*}$-algebras from the perspective of model theory has been undertaken [12]; see also [4, 7-9, 11, 13-16, 24, 25, 40, 41].

The present paper is divided into five sections, besides this introduction. In Section 2 we recall the notion of discrete quantum group, discrete quantum group action, and show that the class of actions of a given discrete quantum group on $\mathrm{C}^{*}$-algebras is first-order axiomatizable. The same is done in Section 3 for compact quantum groups. Section 4 contains some results, to be used in the following sections, relating the notion of ultraproduct of $G$-C $C^{*}$-algebras with crossed products and stabilization. In Section 5 we introduce the notion of positive existential embeddings and Rokhlin property for a $G$-C $\mathrm{C}^{*}$-algebra, generalizing notions introduced in [2] when $G$ is coexact and second countable. The main results of [2] are then generalized to the case of an arbitrary compact quantum group $G$. Finally, Section 6 contains the notion of $G$-equivariant order zero dimension for morphisms between $G$-C $\mathrm{C}^{*}$-algebras, which is used to define the Rokhlin dimension of a $G$-C $\mathrm{C}^{*}$-algebra. Our main preservation results for nuclear dimension and decomposition rank for $G$-C $C^{*}$-algebras with finite Rokhlin dimension are presented here.

Given a subset $X$ of a Banach space $E$, we denote by $[X]$ the closure of the linear span of $X$ inside $E$. If $A$ is a $C^{*}$-algebra, then we let $M(A)$ be its multiplier algebra, and by $\tilde{A}$ its minimal unitization. We canonically identify $A$ with an essential ideal of $M(A)$ and of $\tilde{A}$. We denote by $\otimes$ the injective tensor product of Banach spaces, and the minimal tensor product of $\mathrm{C}^{*}$-algebras (which indeed coincides with the injective tensor product as Banach spaces). The algebraic tensor product of complex algebras is denoted in this paper by $\odot . \mathrm{A}^{*}$-homomorphism $\pi: A \rightarrow B$ between $\mathrm{C}^{*}$-algebras is said to be nondegenerate if $[\pi(A) B]=B$. Given a nondegenerate ${ }^{*}$-homomorphism $\pi: A \rightarrow M(B)$, we also denote by $\pi$ its unique extension to a (unital, strictly continuous) *-homomorphism $\pi: M(A) \rightarrow M(B)$. We say that a $\mathrm{C}^{*}$-subalgebra $A$ of $B$ is nondegenerate if the inclusion map from $A$ to $B$ is nondegenerate. We will frequently use in the following that if $A$ is a $\mathrm{C}^{*}$-algebra, and $I$ is a dense two-sided ideal in $A$, then $I$ contains an increasing approximate unit for $A$.

Given a Hilbert space $\mathcal{H}$, we let $\mathbb{B}(\mathcal{H})$ be the space of bounded linear operators on $\mathcal{H}$, and $\mathbb{K}(\mathcal{H})$ be the space of compact operators on $\mathcal{H}$. Given vectors $\xi, \eta \in \mathcal{H}$ we denote by $\phi_{\xi, \eta}$ the corresponding vector linear functional $\phi_{\xi, \eta}(T):=\langle\eta, T \xi\rangle$. We will frequently use the leg notation for elements in a tensor product [47, Notation 7.1.1]. For a Hilbert space $\mathcal{H}$, we let $\Sigma \in \mathbb{B}(\mathcal{H} \otimes \mathcal{H})$ denote the flip unitary. If $T \in \mathbb{B}(\mathcal{H} \otimes \mathcal{H})$, we let $T_{12}, T_{23}, T_{13} \in \mathbb{B}(\mathcal{H} \otimes \mathcal{H} \otimes \mathcal{H})$ be defined by $T_{12}=T \otimes \operatorname{id}_{\mathcal{H}}, T_{23}=\operatorname{id}_{\mathcal{H}} \otimes T$, and $T_{13}=\Sigma_{23} T_{12} \Sigma_{23}=\Sigma_{12} T_{23} \Sigma_{12}$. 
More generally, one can similarly define the operators $T_{i_{1} \ldots i_{k}} \in B(\mathcal{H} \otimes \cdots \otimes \mathcal{H})$ for any sequence of indices $i_{1}, \ldots, i_{k}$.

\section{An AXIOMATIZATION OF DiscRETE QUANTUM GROUP ACTIONS}

2.1. Discrete quantum groups. A discrete quantum group $G$ is a $\mathrm{C}^{*}$-algebra $c_{0}(G)$ which is a direct sum of full matrix algebras endowed with a nondegenerate *-homomorphism $\Delta: c_{0}(G) \rightarrow M\left(c_{0}(G) \otimes c_{0}(G)\right)($ comultiplication) such that:

- $(\Delta \otimes$ id $) \circ \Delta=($ id $\otimes \Delta) \circ \Delta$;

- $\left[\left(c_{0}(G) \otimes 1\right) \Delta\left(c_{0}(G)\right)\right]=\left[\left(1 \otimes c_{0}(G)\right) \Delta\left(c_{0}(G)\right)\right]=c_{0}(G) \otimes c_{0}(G)$.

The discrete quantum group $G$ is said to be second countable if $c_{0}(G)$ is separable.

Let $G$ be a discrete quantum group. Then there exist an index set $\Lambda$ and finite dimensional Hilbert spaces $\mathcal{H}_{\lambda}$, for $\lambda \in \Lambda$, such that $c_{0}(G)$ is isomorphic to $\bigoplus_{\lambda \in \Lambda} \mathbb{K}\left(\mathcal{H}_{\lambda}\right)$. Set $c_{0}(G)_{\lambda}=\mathbb{K}\left(\mathcal{H}_{\lambda}\right)$ for $\lambda \in \Lambda$, and denotes its unit by $1_{\lambda}$. We identify $c_{0}(G)_{\lambda}$ with a subalgebra of $c_{0}(G), M\left(c_{0}(G)\right)$ with $\prod_{\lambda \in \Lambda} c_{0}(G)_{\lambda}$, and $M\left(c_{0}(G) \otimes c_{0}(G)\right)$ with $\prod_{\mu, \nu \in \Lambda}\left(c_{0}(G)_{\mu} \otimes c_{0}(G)_{\nu}\right)$.

For $\lambda, \mu, \nu \in \Lambda$, we fix a homomorphism $\Delta_{\mu, \nu}^{\lambda}: c_{0}(G)_{\lambda} \rightarrow c_{0}(G)_{\mu} \otimes c_{0}(G)_{\nu}$ satisfying

$$
\Delta(x)=\left(\Delta_{\mu, \nu}^{\lambda}(x)\right)_{\mu, \nu \in \Lambda} \in \prod_{\mu, \nu \in \Lambda} c_{0}(G)_{\mu} \otimes c_{0}(G)_{\nu}
$$

for every $x \in c_{0}(G)_{\lambda}$.

Remark 2.1. Let $\mu, \nu \in \Lambda$, and set $\Lambda_{\mu, \nu}=\left\{\lambda \in \Lambda: \Delta_{\mu, \nu}^{\lambda} \neq 0\right\}$. By [49, Proposition 2.2], the set $\Lambda_{\mu, \nu}$ is finite.

Since $\Delta$ is nondegenerate, we have

$$
\sum_{\lambda \in \Lambda_{\mu, \nu}} \Delta_{\mu, \nu}^{\lambda}\left(1_{\lambda}\right)=1_{\mu} \otimes 1_{\nu}
$$

for every $\mu, \nu \in \Lambda$. Therefore the canonical extension of $\Delta$ to a unital *-homomorphism

$$
\Delta: \prod_{\lambda \in \lambda} c_{0}(G)_{\lambda} \rightarrow \prod_{\mu, \nu \in \Lambda} c_{0}(G)_{\mu} \otimes c_{0}(G)_{\nu}
$$

is defined by

$$
\Delta\left(\left(x_{\lambda}\right)_{\lambda \in \Lambda}\right)=\left(\sum_{\lambda \in \Lambda_{\mu, \nu}} \Delta_{\mu, \nu}^{\lambda}(x)\right)_{\mu, \nu \in \Lambda} \in \prod_{\mu, \nu \in \Lambda} c_{0}(G)_{\mu} \otimes c_{0}(G)_{\nu} .
$$

2.2. Discrete quantum group actions. Let $G$ be a discrete group, and let $A$ be a $\mathrm{C}^{*}$-algebra.

Definition 2.2. A (left) action of $G$ on $A$ is an injective nondegenerate *-homomorphism $\alpha: A \rightarrow M\left(c_{0}(G) \otimes A\right)$ such that:

(1) $(\Delta \otimes$ id $) \circ \alpha=($ id $\otimes \alpha) \circ \alpha$ (action condition);

(2) $\left[\left(c_{0}(G) \otimes 1\right) \alpha(A)\right]=c_{0}(G) \otimes A$ (density condition).

We will refer to a $\mathrm{C}^{*}$-algebra $A$ endowed with a distinguished action of $G$ as a $G$-C*-algebra. If $(A, \alpha)$ and $(B, \beta)$ are $G$-C $\mathrm{C}^{*}$-algebras, then a ${ }^{*}$-homomorphism $\phi: A \rightarrow B$ is said to be $G$-equivariant if it satisfies (id $\otimes \phi) \circ \alpha=\beta \circ \phi$. It follows from the density condition that, if $\left(u_{i}\right)$ is an approximate unit for $A$, then $\left(\alpha\left(u_{i}\right)\right.$ ) is an approximate unit for $c_{0}(G) \otimes A$.

Let $\alpha: A \rightarrow M\left(c_{0}(G) \otimes A\right)$ be a nondegenerate injective ${ }^{*}$-homomorphism. For $\lambda \in \Lambda$, let $\alpha_{\lambda}: A \rightarrow c_{0}(G)_{\lambda} \otimes A$ be the coordinate function for $\alpha$, so that

$$
\alpha(a)=\left(\alpha_{\lambda}(a)\right)_{\lambda \in \Lambda} \in \prod_{\lambda \in \Lambda} c_{0}(G)_{\lambda} \otimes A .
$$

The conditions from Definition 2.2 can be restated as

(1') $\left(\operatorname{id}_{c_{0}(G)_{\mu}} \otimes \alpha_{\nu}\right) \circ \alpha_{\mu}=\sum_{\lambda \in \Lambda_{\mu, \nu}}\left(\Delta_{\mu, \nu}^{\lambda} \otimes \mathrm{id}_{A}\right) \circ \alpha_{\lambda}$ for every $\mu, \nu \in \Lambda$;

(2') $\left[\left(c_{0}(G)_{\lambda} \otimes 1\right) \alpha_{\lambda}(A)\right]=c_{0}(G)_{\lambda} \otimes A$ for every $\lambda \in \Lambda$.

In turn, by Cohen's factorization theorem, (2') is equivalent to the assertion that for every $\lambda \in \Lambda$, for every $a \in c_{0}(G)_{\lambda} \otimes A$ of norm at most 1 , and every $\varepsilon>0$, there exist $x \in c_{0}(G)_{\lambda}$ and $b \in A$ of norm at most 1 such that $\|(x \otimes 1) \alpha(b)-a\|<\varepsilon$. 
Example 2.3. If $G$ is a classical discrete group, then one can regard $G$ as a discrete quantum group by considering the $C^{*}$-algebra $c_{0}(G)$ of functions from $G$ to $\mathbb{C}$ vanishing at infinity. In this case, one has that $\Lambda=G$ and $c_{0}(G)_{\lambda}=\mathbb{C}$ for every $\lambda \in G$. The comultiplication function $\Delta: c_{0}(G) \rightarrow M\left(c_{0}(G) \otimes c_{0}(G)\right)$ is defined by setting $\Delta_{\mu \nu}^{\lambda}(1)=\delta_{\lambda, \mu \nu}$ for $\lambda, \mu, \nu \in G$.

Every discrete quantum group $G$ such that $c_{0}(G)$ is a commutative $C^{*}$-algebra arises from a classical discrete group in this fashion.

2.3. Axiomatization. We continue to fix a discrete quantum group $G$. We now describe a natural (multisorted) language $\mathcal{L}_{G}^{\mathrm{C}^{*}}$ that has $G$-C $\mathrm{C}^{*}$-algebras as structures. For comparison, one can refer to the language considered in the axiomatization of operator systems from [24, Appendix B].

2.3.1. The language. The language $\mathcal{L}_{G}^{\mathrm{C}^{*}}$ has, for every $n \geq 1$, sorts $\mathcal{S}^{(n)}$ and $\mathcal{C}^{(n)}$ to be interpreted as $M_{n}(\mathbb{C}) \otimes A$ and $M_{n}(\mathbb{C})$, respectively. For each of these, the domains of quantifications should be interpreted as the balls with respect to the norm centered at the origin.

The function and relation symbols consist of:

(1) function and relation symbols for the $\mathrm{C}^{*}$-algebra operations and the $\mathrm{C}^{*}$-algebra norm on $M_{n}(\mathbb{C}) \otimes A$ and $M_{n}(\mathbb{C})$ for every $n \in \mathbb{N}$;

(2) function symbols for the $M_{n}(\mathbb{C})$-bimodule structure on $M_{n}(\mathbb{C}) \otimes A$;

(3) constant symbols the elements of $M_{n}(\mathbb{C})$;

(4) for any bounded linear map $T: M_{n}(\mathbb{C}) \rightarrow M_{m}(\mathbb{C})$, function symbols $\mathcal{C}^{(n)} \rightarrow \mathcal{C}^{(m)}$ and $\mathcal{S}^{(n)} \rightarrow \mathcal{S}^{(m)}$ to be interpreted as $T$ and $T \otimes \mathrm{id}_{A}$;

(5) function symbols for the canonical inclusions of each of the tensor factors in $M_{n}(\mathbb{C}) \otimes A$;

(6) function symbols $\mathcal{S} \rightarrow \mathcal{S}^{\left(d_{\lambda}\right)}$ to be interpreted as the injective ${ }^{*}$-homomorphisms $\alpha_{\lambda}: A \rightarrow c_{0}(G)_{\lambda} \otimes A$ that define the action.

It is clear that any $G$-C $\mathrm{C}^{*}$-algebra can be seen as an $\mathcal{L}_{G}^{\mathrm{C} *}$-structure in a canonical way.

2.3.2. The axioms. We now describe axioms for the class of $G$-C*-algebras in the language $\mathcal{L}_{G}^{\mathrm{C}^{*}}$ described above. Such axioms are designed to guarantee the following:

(1) the interpretations of the sort $\mathcal{S}^{(n)}$ and $\mathcal{C}^{(n)}$ are $\mathrm{C}^{*}$-algebras;

(2) the domains of quantifications are interpreted as the balls (see [12, Example 2.2.1]);

(3) the sorts $\mathcal{S}^{(n)}$ and $\mathcal{C}^{(n)}$, the symbols for the canonical inclusions of each of the tensor factors in $M_{n}(\mathbb{C}) \otimes A$, the symbols for the maps $T$ and $T \otimes \mathrm{id}_{A}$ for any bounded linear map $T: M_{n}(\mathbb{C}) \rightarrow M_{m}(\mathbb{C})$, the symbols for the $M_{n}(\mathbb{C})$-bimodule structure on $M_{n}(\mathbb{C}) \otimes A$, and the symbols for the elements of $M_{n}(\mathbb{C})$, are interpreted as they should be (see [24, Appendix C]);

(4) the interpretation of the symbols for the maps $\alpha_{\lambda}$ that define the action are isometric *-homomorphisms;

(5) for every $\mu, \nu \in \Lambda$,

$$
\left(\operatorname{id}_{c_{0}(G)_{\mu}} \otimes \alpha_{\nu}\right) \circ \alpha_{\mu}=\sum_{\lambda \in \Lambda_{\mu, \nu}}\left(\Delta_{\mu, \nu}^{\lambda} \otimes \mathrm{id}_{A}\right) \circ \alpha_{\lambda}
$$

(6) for every $a \in c_{0}(G)_{\lambda} \otimes A$ of norm at most 1 , and every $\varepsilon>0$, there exist $x \in c_{0}(G)_{\lambda}$ and $b \in A$, both of norm at most 1 , such that $\|(x \otimes 1) \alpha(b)-a\|<\varepsilon$.

It is clear from the discussion above that these axioms indeed axiomatize the class of $G$-C*algebras. Furthermore, it is easy to see that these axioms are all given by conditions of the form $\sigma \leq r$ where $\sigma$ is a positive primitive $\forall \exists-\mathcal{L}_{G}^{\mathrm{C}^{*}}$-sentence and $r \in \mathbb{R}$. Therefore, this shows that the class of $G$-C $\mathrm{C}^{*}$-algebras is positively primitively $\forall \exists$-axiomatizable in the language $\mathcal{L}_{G}^{\mathrm{C} *}$ in the sense of Definition A.3. One can observe that, when $G$ is second-countable, the language $\mathcal{L}_{G}^{\mathrm{C}^{*}}$ is separable for the class of $G$-C $\mathrm{C}^{*}$-algebras; see Definition A.1. More generally, the density character of $\mathcal{L}_{G}^{\mathrm{C}^{*}}$ for the class of $G$-C*-algebras is equal to the size of $\Lambda$. It is not difficult to verify that an $\mathcal{L}_{G}^{\mathrm{C}^{*}}$-morphism between $G$-C $\mathrm{C}^{*}$-algebras is precisely a $G$-equivariant *-homomorphism, while an $\mathcal{L}_{G}^{\mathrm{C}^{*}}$-embedding is an injective $G$-equivariant *-homomorphism.

2.3.3. Ultraproducts. Once $G$-C $\mathrm{C}^{*}$-algebras are regarded as $\mathcal{L}_{G}^{\mathrm{C}^{*}}$-structures as described above, one can consider ultraproducts of $G$-C $\mathrm{C}^{*}$-algebras as a particular instance of ultraproducts in first-order logic for metric structures;

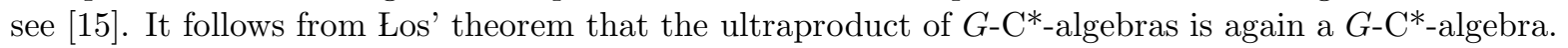

More generally, one can consider reduced products with respect to an arbitrary filter $\mathcal{F}$ as defined in [23]. It is easy to see that, in the particular case when $\mathcal{F}$ is the filter of cofinite subsets of $\mathbb{N}$, then the reduced power of a $G$-C $\mathrm{C}^{*}$-algebra $(A, \alpha)$ with respect to $\mathcal{F}$ coincides with the $G$-C ${ }^{*}$-algebra $\left(A_{\infty}, \alpha_{\infty}\right)$ constructed in [2]. It follows from the fact that the class of $G$-C $C^{*}$-algebras is positively primitively $\forall \exists$-axiomatizable in the language 
$\mathcal{L}_{G}^{\mathrm{C}^{*}}$ together with Corollary A.6 that the reduced product of $G$-C*-algebras is again a $G$-C*-algebra. This recovers [2, Lemma 2.6] as a particular case.

2.3.4. Other languages. Occasionally, it is useful to consider $\mathrm{C}^{*}$-algebras as structures in a language other than the standard language for $\mathrm{C}^{*}$-algebras $\mathcal{L}^{\mathrm{C}^{*}}$. These other languages are useful to capture properties that are preserved by more general classes of morphisms, rather than just *-homomorphisms, such as for example completely positive contractive maps or completely positive contractive order zero maps. Several languages are considered in [22, Section 3]. For each such language $\mathcal{L}$, one can consider a corresponding $G$-equivariant language $\mathcal{L}_{G}$. This can defined as in Subsubsection 2.3.4, by starting with the language $\mathcal{L}$ rather than $\mathcal{L}^{\mathrm{C}^{*}}$.

\section{An axiomatization of COMPACT quAntum GROUP ACtions}

3.1. Compact quantum groups. A (reduced, $\mathrm{C}^{*}$-algebraic) compact quantum group $G$ is given by a unital $\mathrm{C}^{*}$-algebra $C(G)$ endowed with a unital *-homomorphism (comultiplication) $\Delta: C(G) \rightarrow C(G) \otimes C(G)$ and a faithful state $h: C(G) \rightarrow \mathbb{C}$ (the Haar state) satisfying

- $(\Delta \otimes$ id $) \circ \Delta=($ id $\otimes \Delta) \circ \Delta$,

- $[\Delta(C(G))(1 \otimes C(G))]=[\Delta(C(G))(C(G) \otimes 1)]=C(G) \otimes C(G)$, and

- $(h \otimes$ id $) \circ \Delta=($ id $\otimes h) \circ \Delta=h$

where in the last equation we identify $\mathbb{C}$ with the space of scalar multiples for the identity in $C(G)$. The first two conditions assert that $C(G)$ endowed with the comultiplication $\Delta$ is a unital Hopf $\mathrm{C}^{*}$-algebra [43, Definition 2.2]. A compact quantum group is said to be second countable if $C(G)$ is separable.

A unitary representation of $G$ on a Hilbert space $\mathcal{H}$ is a unitary $u \in M(C(G) \otimes \mathbb{K}(\mathcal{H}))$ satisfying $(\Delta \otimes \mathrm{id})(u)=$ $u_{13} u_{23}$. In the following we will only consider the case when $\mathcal{H}$ is finite-dimensional, in which case one has that $u \in C(G) \otimes \mathbb{B}(\mathcal{H})$. We also identify the unitary representation $u$ of $G$ on $\mathcal{H}$ with the linear map $\mathcal{H} \rightarrow C(G) \otimes \mathcal{H}$ given by $\eta \mapsto u(1 \otimes \eta)$ for all $\eta \in \mathcal{H}$. A subspace $\mathcal{K} \subseteq \mathcal{H}$ is invariant if $u(1 \otimes \mathcal{K}) \subseteq C(G) \otimes \mathcal{K}$. Direct sum and tensor product of unitary representations is defined in the usual way. A unitary representation is called irreducible if it has no non-trivial invariant closed subspace. Every irreducible unitary representation of $G$ is finite-dimensional, and every unitary representation of $G$ is equivalent to a direct sum of irreducible unitary representations.

We let $\operatorname{Rep}(G)$ be the set of unitary representations of $G$ on finite-dimensional Hilbert spaces. For $\lambda \in \operatorname{Rep}(G)$ we let $u^{\lambda} \in C(G) \otimes \mathbb{K}\left(\mathcal{H}_{\lambda}\right)$ be the associated representation, and we denote by $d_{\lambda}$ the dimension of $\mathcal{H}_{\lambda}$. We also define $\operatorname{Irr}(G)$ to be the set of equivalence classes of irreducible unitary representations of $G$. Given $\lambda, \mu \in \operatorname{Irr}(G)$, we set $\delta_{\lambda, \mu}=1$ if $\lambda$ and $\mu$ are equivalent, and $\delta_{\lambda, \mu}=0$ otherwise. For $\lambda \in \operatorname{Rep}(G)$ and $\xi, \eta \in \mathcal{H}_{\lambda}$, set $u_{\xi, \eta}^{\lambda}=\left(\mathrm{id} \otimes \phi_{\xi, \eta}\right)\left(u^{\lambda}\right) \in C(G)$. These are called the matrix coefficients of the unitary representation $\lambda$. If $\lambda \in \operatorname{Irr}(G)$, then we fix an orthonormal basis $\left\{e_{k}^{\lambda}\right\}_{k=1}^{d_{\lambda}}$ of $\mathcal{H}_{\lambda}$ and set $u_{j k}^{\lambda}=u_{e_{j}, e_{k}}^{\lambda}$ for $1 \leq j, k \leq d_{\lambda}$. For an arbitrary $\lambda \in \operatorname{Rep}(G)$, we write $\lambda$ as a direct sum of irreducible unitary representations $\lambda_{1} \oplus \cdots \oplus \lambda_{n}$, and then we consider the orthonormal basis of $\mathcal{H}_{\lambda}=\mathcal{H}_{\lambda_{1}} \oplus \cdots \oplus \mathcal{H}_{\lambda_{n}}$ associated with the given orthonormal bases of $\mathcal{H}_{\lambda_{1}}, \ldots, \mathcal{H}_{\lambda_{n}}$.

Define $\mathcal{O}(G)$ to be the dense selfadjoint subalgebra of $C(G)$ given by

$$
\mathcal{O}(G)=\left\{u_{\xi, \eta}^{\lambda}: \lambda \in \operatorname{Rep}(G), \xi, \eta \in \mathcal{H}_{\lambda}\right\}=\operatorname{span}\left\{u_{i j}^{\lambda}: \lambda \in \operatorname{Irr}(G), 1 \leq i, j \leq d_{\lambda}\right\}
$$

and observe that it is invariant under the comultiplication. The induced *-bialgebra structure on $\mathcal{O}(G)$ turns it into a Hopf *-algebra [47, Definition 1.3.24]. The counit map $\epsilon$ on $\mathcal{O}(G)$ is defined by $\epsilon\left(u_{\xi, \eta}^{\lambda}\right)=\langle\xi, \eta\rangle$, while the antipode map $S$ is given by $S\left(u_{\xi, \eta}^{\lambda}\right)=\left(u_{\eta, \xi}^{\lambda}\right)^{*}$, for $\xi, \eta \in \mathcal{H}_{\lambda}$ and $\lambda \in \operatorname{Rep}(G)$.

Remark 3.1. The $\mathrm{C}^{*}$-algebra $C(G)$ together with its comultiplication can be recovered from $\mathcal{O}(G)$ as the closure of the image of $\mathcal{O}(G)$ in the GNS representation induced by the restriction of the Haar state to $\mathcal{O}(G)$.

By [6, Theorem 1.8], for every $\lambda \in \operatorname{Irr}(G)$ there is a positive invertible operator $F_{\lambda} \in \mathbb{K}\left(\mathcal{H}_{\lambda}\right)$ satisfying:

$$
h\left(u_{\xi, \eta}^{\lambda}\left(u_{\zeta, \chi}^{\lambda}\right)^{*}\right)=\frac{\langle\xi, \zeta\rangle\left\langle\chi, F_{\lambda} \eta\right\rangle}{\operatorname{dim}_{q}(\lambda)} \text { and } h\left(\left(u_{\xi, \eta}^{\lambda}\right)^{*} u_{\zeta, \chi}^{\lambda}\right)=\frac{\left\langle\xi,\left(F_{\lambda}\right)^{-1} \zeta\right\rangle\langle\chi, \eta\rangle}{\operatorname{dim}_{q}(\lambda)},
$$

for all $\xi, \eta \in \mathcal{H}_{\lambda}$, where $\operatorname{dim}_{q}(\lambda)=\operatorname{Tr}\left(F_{\lambda}\right)$ is the quantum dimension of $\lambda$. Furthermore, one has

$$
h\left(u_{\xi, \eta}^{\lambda}\left(u_{\zeta, \chi}^{\mu}\right)^{*}\right)=h\left(\left(u_{\xi, \eta}^{\lambda}\right)^{*} u_{\zeta, \chi}^{\mu}\right)=0
$$

whenever $\lambda, \mu$ are nonequivalent, for all $\xi, \eta \in \mathcal{H}_{\lambda}$, and all $\zeta, \chi \in \mathcal{H}_{\mu}$. In particular, we deduce that

$$
h\left(u_{i j}^{\lambda}\left(u_{k \ell}^{\mu}\right)^{*}\right)=\delta_{\lambda \mu} \delta_{i k} \frac{F_{\ell j}^{\lambda}}{\operatorname{dim}_{q}(\lambda)} .
$$


for $\lambda, \mu \in \operatorname{Irr}(G), 1 \leq i, j \leq d_{\lambda}$, and $1 \leq k, \ell \leq d_{\mu}$. Similarly to the classical case, to each $\lambda, \mu \in \operatorname{Irr}(G)$ there corresponds a contragradient representation $\bar{\lambda} \operatorname{Irr}(G)$ (cf. [39, Section 6]).

For $\lambda \in \operatorname{Rep}(G)$, set

$$
C(G)_{\lambda}=\left\{u_{\xi, \eta}^{\lambda}: \xi, \eta \in \mathcal{H}_{\lambda}\right\}=\operatorname{span}\left\{u_{i j}^{\lambda}: 1 \leq i, j \leq d_{\lambda}\right\}
$$

Then $C(G)_{\lambda}$ is a finite-dimensional subspace of $C(G)$ which is invariant under the comultiplication. If $\lambda, \mu \in$ $\operatorname{Irr}(G)$, then $C(G)_{\lambda}$ and $C(G)_{\mu}$ are orthogonal with respect to the inner product defined by the Haar state $h$. It follows that $\mathcal{O}(G)$ is equal to the algebraic direct sum of $C(G)_{\lambda}$ for $\lambda \in \operatorname{Irr}(G)$. For $\lambda, \mu \in \operatorname{Rep}(G)$, we have

$$
C(G)_{\lambda}+C(G)_{\mu}=C(G)_{\lambda \oplus \mu}, \quad \operatorname{span}\left(C(G)_{\lambda} C(G)_{\mu}\right)=C(G)_{\lambda \otimes \mu}, \text { and }\left(C(G)_{\lambda}\right)^{*}=C(G)_{\bar{\lambda}} .
$$

Define $\mathcal{O}(\hat{G})$ to be the space of linear functionals on $C(G)$ of the form $x \mapsto h(x y)$ for some $y \in \mathcal{O}(G)$. For $\lambda \in \operatorname{Rep}(G)$ and $1 \leq s, m \leq d_{\lambda}$, let $\omega_{s m}^{\lambda} \in \mathcal{O}(\hat{G})$ be given by

$$
\omega_{s m}^{\lambda}(x)=\operatorname{dim}_{q}(\lambda) \sum_{k=1}^{d_{\lambda}}\left(F_{\lambda}\right)_{m k}^{-1} h\left(x\left(u_{s k}^{\lambda}\right)^{*}\right) .
$$

It follows from Equation 1 that

$$
\omega_{s m}^{\lambda}\left(u_{l n}^{\mu}\right)=\delta_{\mu \lambda} \delta_{l s} \delta_{m n} \text { and }\left(\omega_{s m}^{\lambda} \otimes \omega_{l n}^{\mu}\right) \circ \Delta=\delta_{m l} \delta_{\mu \nu} \omega_{s n}^{\mu}
$$

for $\lambda, \mu \in \operatorname{Irr}(G), 1 \leq s, m \leq d_{\lambda}$, and $1 \leq l, n \leq d_{\lambda}$. More generally, one can define

$$
\omega_{\xi, \eta}^{\lambda}=\operatorname{dim}_{q}(\lambda) \sum_{k=1}^{d_{\lambda}}\left\langle\left(F_{\lambda}\right)^{-1} e_{k}, \eta\right\rangle h\left(x\left(u_{\xi, e_{k}}^{\lambda}\right)^{*}\right)
$$

for $\xi, \eta \in \mathcal{H}_{\lambda}$, and observe that $\left(\omega_{\xi, \eta}^{\lambda} \otimes \omega_{\zeta, \chi}^{\lambda}\right) \circ \Delta=\langle\eta, \zeta\rangle \omega_{\xi, \chi}^{\lambda}$ for every $\xi, \eta, \zeta, \chi \in \mathcal{H}_{\lambda}$.

Set $P_{k s}^{\lambda}=\left(\omega_{k s}^{\lambda} \otimes \mathrm{id}\right) \circ \Delta$ for $1 \leq k, s \leq d_{\lambda}$. From the relations above, it follows that $P_{s m}^{\lambda} P_{l n}^{\mu}=\delta_{\lambda \mu} \delta_{m l} P_{s n}^{\lambda}$ for $\lambda, \mu \in \operatorname{Irr}(G), 1 \leq s, m \leq d_{\lambda}$, and $1 \leq l, n \leq d_{\mu}$. Furthermore, $P_{k k}^{\lambda}$ is a projection operator onto

$$
C(G)_{\lambda, k}:=\operatorname{span}\left\{u_{i k}^{\lambda}: 1 \leq i \leq d_{\lambda}\right\} \text {, }
$$

and $P^{\lambda}:=\sum_{k=1}^{d_{\lambda}} P_{k k}^{\lambda}$ is a projection onto $C(G)_{\lambda}$.

3.2. The fundamental unitaries. We now recall the important concept of fundamental unitaries associated with a given compact quantum group $G$.

Definition 3.2. The fundamental unitaries associated with $G$ are the unitary operators $V, W \in \mathbb{B}\left(L^{2}(G) \otimes\right.$ $\left.L^{2}(G)\right)$ defined as follows.

- The operator $W$ is the adjoint of the unitary operator induced by the linear map with dense image $C(G) \otimes C(G) \rightarrow C(G) \otimes C(G)$ given by $x \otimes y \mapsto \Delta(y)(x \otimes 1)$.

- Similarly, $V$ is the unitary operator induced by the linear map with dense image $C(G) \otimes C(G) \rightarrow$ $C(G) \otimes C(G)$ given by $x \otimes y \mapsto \Delta(x)(1 \otimes y)$.

Abbreviate $\mathbb{K}\left(L^{2}(G)\right)$ to $\mathbb{K}_{G}$, and identify the Banach-space dual $\mathbb{K}_{G}^{*}$ with the predual of $\mathbb{B}\left(L^{2}(G)\right)$. The fundamental unitaries satisfy the pentagon equations

$$
V_{12} V_{13} V_{23}=V_{23} V_{12} \quad \text { and } \quad W_{12} W_{13} W_{23}=W_{23} W_{12}
$$

The $\mathrm{C}^{*}$-algebra $C(G) \subseteq \mathbb{B}\left(L^{2}(G)\right)$ can be recovered as the left leg of $W$ :

$$
C(G)=\left[\left\{\left(\mathrm{id} \otimes \phi_{\xi, \xi^{\prime}}\right) W: \xi, \xi^{\prime} \in L^{2}(G)\right\}\right]=\left[\left\{(\mathrm{id} \otimes \omega)(W): \omega \in \mathbb{K}_{G}^{*}\right\}\right] .
$$

Similarly, the group $\mathrm{C}^{*}$-algebra $C^{*}(G)=c_{0}(\hat{G})$ can be recovered as the right leg of $W$ :

$$
C^{*}(G)=\left[\left\{\left(\phi_{\xi, \xi^{\prime}} \otimes \mathrm{id}\right)(W): \xi, \xi^{\prime} \in L^{2}(G)\right\}\right]=\left[\left\{(\omega \otimes \mathrm{id})(W): \omega \in \mathbb{K}_{G}^{*}\right\}\right] .
$$

One can recover $C(G)$ as the right leg of $V$, and $c_{0}(\hat{G})$ as the left leg of $V$. Furthermore, $W$ belongs to $M\left(C(G) \otimes c_{0}(\hat{G})\right)$ and $V$ belongs to $M\left(c_{0}(\hat{G}) \otimes C(G)\right)$.

Define normal *-homomorphisms $\Delta, \hat{\Delta}: \mathbb{B}\left(L^{2}(G)\right) \rightarrow \mathbb{B}\left(L^{2}(G) \otimes L^{2}(G)\right)$ by

$$
\Delta(x)=W^{*}(1 \otimes x) W \quad \text { and } \quad \hat{\Delta}(x)=\hat{W}^{*}(1 \otimes y) \hat{W},
$$

for all $x, y \in \mathbb{B}\left(L^{2}(G)\right)$. The restriction of $\Delta$ to $C(G)$ gives the comultiplication of $C(G)$, while the restriction of $\hat{\Delta}$ to $c_{0}(\hat{G})$ gives the comultiplication of $c_{0}(\hat{G})$. 
3.3. Compact quantum group actions. Let $G$ be a (reduced, $\mathrm{C}^{*}$-algebraic) compact quantum group, and let $A$ be a $\mathrm{C}^{*}$-algebra.

Definition 3.3. A (left, continuous) action of $G$ on $A$ is an injective nondegenerate ${ }^{*}$-homomorphism $\alpha: A \rightarrow$ $C(G) \otimes A$ satisfying the following conditions:

(1) $(\Delta \otimes \mathrm{id}) \circ \alpha=(\mathrm{id} \otimes \alpha) \circ \alpha($ action condition);

(2) $[(C(G) \otimes 1) \alpha(A)]=C(G) \otimes A$, where $C(G) \otimes 1, \alpha(G)$, and $C(G) \otimes A$ are canonically regarded as subalgebras of $M(C(G) \otimes A)$ (density condition).

If $A$ is a $\mathrm{C}^{*}$-algebra endowed with a distinguished action $\alpha$ of $G$ on $A$, then we refer to the pair $(A, \alpha)$ as a $G$-C*-algebra. A canonical example of $G$-C $C^{*}$-algebra is $C(G)$ endowed with the left translation action of $G$ on $C(G)$ given by the comultiplication $\Delta$.

Suppose now that $\lambda \in \operatorname{Rep}(G)$. An intertwiner between $\lambda$ and $\alpha$ is a linear map $v: \mathcal{H}_{\lambda} \rightarrow A$ such that

$$
\alpha(v \xi)=(\mathrm{id} \otimes v) u^{\lambda}(1 \otimes \xi)
$$

for every $\xi \in \mathcal{H}_{\lambda}$. The space of intertwiners between $\lambda$ and $\alpha$ is denoted by $\operatorname{Int}(\lambda, \alpha)$. The $\lambda$-isotypical component (or $\lambda$-spectral subspace) is the closed subspace

$$
A_{\lambda}=\left\{v \xi: \xi \in \mathcal{H}_{\lambda}, v \in \operatorname{Int}(\lambda, \alpha)\right\} \text {. }
$$

Define $E_{k s}^{\lambda}: A \rightarrow A_{\lambda}$ as $E_{k s}^{\lambda}=\left(\omega_{k s}^{\lambda} \otimes\right.$ id $) \circ \alpha$ for $1 \leq k, s \leq d_{\lambda}$. It follows from the Schur orthogonality relations (1) that $E_{s m}^{\lambda} E_{l n}^{\mu}=\delta_{\lambda \mu} \delta_{m l} E_{s n}$ for $\lambda, \mu \in \operatorname{Irr}(G)$. The proof of [45, Theorem 1.5] shows that $A_{\lambda}$ is a closed subspace of $A$. Indeed, one can alternatively describe $A_{\lambda}$ as

$$
A_{\lambda}=\left\{a \in A: \alpha(a) \in C(G)_{\lambda} \otimes A\right\}=\left\{a \in A: \alpha(a) \in C(G)_{\lambda} \otimes A_{\lambda}\right\} .
$$

Furthermore $A_{\lambda}+A_{\mu} \subseteq A_{\lambda \oplus \mu}, A_{\lambda} A_{\mu} \subseteq A_{\lambda \otimes \mu}$, and $\left(A_{\lambda}\right)^{*}=A_{\bar{\lambda}}$ for every $\lambda, \mu \in \operatorname{Rep}(G)$.

It is also shown in [45, Theorem 1.5] that $E^{\lambda}=\sum_{k=1}^{d_{\lambda}} E_{k k}^{\lambda}$ is a projection onto $A_{\lambda}$. Moreover, $E_{k k}^{\lambda}$ is a projection onto a closed subspace $A_{\lambda, k}$ of $A_{\lambda}$ of dimension $c_{\alpha} \in \mathbb{N} \cup\{\infty\}$. Such a dimension $c_{\alpha}$ is called the multiplicity of $\lambda$ in the spectrum of $\alpha$. Let $\left(a_{1, i}^{\lambda}\right)_{i=1}^{c_{\lambda}}$ be a basis of $A_{\lambda, 1}$. Then $\left(E_{s 1}^{\lambda}\left(a_{1, i}^{\lambda}\right)\right)_{i=1}^{c_{\lambda}}$ is a basis of $A_{\lambda, s}$. For $s=1, \ldots, d_{\lambda}$, set $a_{s, i}^{\lambda}=E_{s 1}^{\lambda}\left(a_{1, i}^{\lambda}\right)$. Then $E_{s k}^{\lambda}\left(a_{k, i}^{\lambda}\right)=a_{s, i}^{\lambda}$, and $\alpha\left(a_{k, i}^{\lambda}\right)=\sum_{s=1}^{d_{\lambda}} u_{k s}^{\lambda} \otimes a_{s, i}^{\lambda}$ for every $k=1,2, \ldots, d_{\lambda}$. From this it easily follows that $(\epsilon \otimes \mathrm{id}) \alpha(c)=c$ for every $c \in A_{\lambda}$. Define $\mathcal{O}(A)=\operatorname{span}\left\{A_{\lambda}: \lambda \in \operatorname{Irr}(A)\right\}$. It is shown in [45, Theorem 1.5] that $\mathcal{O}(A)$ is a dense selfadjoint subalgebra of $A$, called Podles subalgebra or algebraic core of $(A, \alpha)$.

The spectral subspace $A^{\alpha}:=A_{\mathrm{t}}$ associated with the trivial representation $\mathrm{t}$ of $G$ is a nondegenerate $\mathrm{C}^{*}$ subalgebra of $A$, called the fixed point algebra. The projection $E^{\mathrm{t}}$ onto $A^{\alpha}$ is a faithful conditional expectation, and $A_{\lambda}$ is an $A^{\alpha}$-bimodule for every $\lambda \in \operatorname{Rep}(G)$. The formula $\langle a, b\rangle=E^{\mathrm{t}}\left(a^{*} b\right)$ defines a (right) full Hilbert $A^{\alpha}$-module structure on $A\left[6\right.$, Lemma 3.8 and Lemma 3.19], and two spectral subspaces $A_{\lambda}, A_{\mu}$ associated with nonequivalent $\lambda, \mu \in \operatorname{Irr}(G)$ are orthogonal with respect to this $\mathrm{C}^{*}$-bimodule structure.

Lemma 3.4. Let $\lambda \in \operatorname{Rep}(G)$. For $a=\sum_{i j} u_{i j}^{\lambda} \otimes a_{i j} \in C(G)_{\lambda} \otimes A$, one has

$$
\|a\|_{C(G) \otimes A}=\sup \left\{\left\|\sum_{i j} \mu_{i j} a_{i j}\right\|_{A}: \mu_{i j} \in \mathbb{C},\left\|\sum_{i j} \mu_{i j} u_{i j}^{\lambda}\right\|_{C(G)} \leq 1\right\} .
$$

Proof. It is enough to observe that $C(G)_{\lambda} \otimes A$ is endowed with the injective Banach space tensor product, and that $\left\{u_{i j}^{\lambda}: 1 \leq i, j \leq d_{\lambda}\right\}$ is a basis for $C(G)_{\lambda}$.

We close this subsection with the following lemma.

Lemma 3.5. Let $\lambda \in \operatorname{Irr}(G)$.

(1) Let $1 \leq i, j \leq d_{\lambda}$. Then $\left(P_{i j}^{\lambda} \otimes \mathrm{id}\right) \circ \alpha=\alpha \circ E_{i j}^{\lambda}$. Thus, $\left(P^{\lambda} \otimes \mathrm{id}\right) \circ \alpha=\alpha \circ E^{\lambda}$.

(2) Set $E^{\lambda}=\sum_{j=1}^{d_{\lambda}} E_{j j}^{\lambda}$. For $a \in A$, one has

$$
1 \otimes E^{\lambda}(a)=\sum_{1 \leq s, t \leq d_{\lambda}}\left(\left(u_{s t}^{\lambda}\right)^{*} \otimes 1\right)\left(\alpha \circ E_{s t}^{\lambda}\right)(a) .
$$

Proof. Part (1) is straightforward, so we show (2). By linearity, we can assume that $a$ has the form $a=a_{k, i}^{\lambda}$ for some $1 \leq i \leq c_{\lambda}$ and $1 \leq k \leq d_{\lambda}$. Using the fact that $\mathcal{O}(G)$ is a Hopf $*_{\text {-algebra, we have }}$

$$
(m \circ(S \otimes \mathrm{id}) \circ \Delta)(x)=\varepsilon(x) 1
$$


for every $x \in \mathcal{O}(G)$. Therefore

$$
\begin{aligned}
1 \otimes a_{k, i}^{\lambda} & =(\epsilon \otimes \mathrm{id}) \alpha\left(a_{k, i}^{\lambda}\right)=(m \otimes \mathrm{id})(S \otimes \mathrm{id} \otimes \mathrm{id})(\Delta \otimes \mathrm{id}) \alpha\left(a_{k, i}^{\lambda}\right) \\
& =\sum_{1 \leq j \leq d_{\lambda}}(m \otimes \mathrm{id})(S \otimes \mathrm{id} \otimes \mathrm{id})(\Delta \otimes \mathrm{id})\left(u_{k j} \otimes a_{j, i}^{\lambda}\right) \\
& =\sum_{1 \leq j, s \leq d_{\lambda}}(m \otimes \mathrm{id})(S \otimes \mathrm{id} \otimes \mathrm{id})\left(u_{k s}^{\lambda} \otimes u_{s j}^{\lambda} \otimes a_{j, i}^{\lambda}\right) \\
& =\sum_{1 \leq j, s \leq d_{\lambda}}\left(\left(u_{s k}^{\lambda}\right)^{*} u_{s j}^{\lambda} \otimes a_{j, i}^{\lambda}\right) \\
& =\sum_{1 \leq s \leq d_{\lambda}}\left(\left(u_{s k}^{\lambda}\right)^{*} \otimes 1\right)\left(\sum_{j=1}^{d_{\lambda}} u_{s j}^{\lambda} \otimes a_{j, i}^{\lambda}\right) \\
& =\sum_{1 \leq s \leq d_{\lambda}}\left(\left(u_{s k}^{\lambda}\right)^{*} \otimes 1\right) \alpha\left(a_{s, i}^{\lambda}\right) \\
& =\sum_{1 \leq s \leq d_{\lambda}}\left(\left(u_{s k}^{\lambda}\right)^{*} \otimes 1\right)\left(\alpha \circ E_{s k}^{\lambda}\right)\left(a_{k, i}^{\lambda}\right) \\
& =\sum_{1 \leq s, t \leq d_{\lambda}}\left(\left(u_{s t}^{\lambda}\right)^{*} \otimes 1\right)\left(\alpha \circ E_{s t}^{\lambda}\right)\left(a_{k, i}^{\lambda}\right) .
\end{aligned}
$$

This concludes the proof.

3.4. Axiomatization. Throughout this subsection, we fix a compact quantum group $G$. Our goal is to show that there is a natural language $\mathcal{L}_{G}^{\mathrm{C}^{*}}$ in the logic for metric structures that allows one to regard $G$-C $\mathrm{C}^{*}$-algebra as $\mathcal{L}_{G}^{\mathrm{C}^{*}}$-structures, in such a way that the class of $G$-C $\mathrm{C}^{*}$-algebra is axiomatizable. We begin by describing the language, and then present the axioms for the class of $G$-C $\mathrm{C}^{*}$-algebras.

3.4.1. The language. The language $\mathcal{L}_{G}^{\mathrm{C}^{*}}$ has the following sorts:

- a sort $\mathcal{S}$, to be interpreted as the $\mathrm{C}^{*}$-algebra $A$ where $G$ acts;

- a sort $\mathcal{S}^{(0)}$ to be interpreted as $C(G)$;

- a sort $\mathcal{S}^{(1)}$ to be interpreted as $C(G) \otimes A$;

- a sort $\mathcal{C}$ to be interpreted as the algebra of complex numbers.

For each of the sorts above, the domains of quantifications are as follows:

- $\mathcal{S}$ has domains of quantification $D_{n}^{\lambda}$ for $\lambda \in \operatorname{Rep}(G)$ and $n \in \mathbb{N}$, to be interpreted as the ball of radius $n$ of $A_{\lambda} \subseteq A$ centered at the origin;

- $\mathcal{S}^{(0)}$ has domains of quantification $D_{n}^{(0), \lambda}$ for $\lambda \in \operatorname{Rep}(G)$ and $n \in \mathbb{N}$, to be interpreted as the ball of radius $n$ of $C(G)_{\lambda} \subseteq C(G)$ centered at the origin;

- $\mathcal{S}^{(1)}$ has domains of quantification $D_{n}^{(1), \lambda}$ for $\lambda \in \operatorname{Rep}(G)$ and $n \in \mathbb{N}$, to be interpreted as the ball of radius $n$ of $C(G)_{\lambda} \otimes A_{\lambda} \subseteq C(G) \otimes A$ centered at the origin;

- $\mathcal{C}$ has domains of quantification $D_{n}$ of $n \in \mathbb{N}$, to be interpreted as the balls of radius $n$ of $\mathbb{C}$ centered at the origin.

The function and relation symbols consist of:

- function and relation symbols for the $\mathrm{C}^{*}$-algebra operations and $\mathrm{C}^{*}$-algebra norm of $A, C(G), C(G) \otimes A$, and $\mathbb{C}$;

- constant symbols for the elements of $\mathbb{C}$ and for the elements of $\mathcal{O}(G)$;

- function symbols for the $\mathcal{O}(G)$-bimodule structure of $C(G) \otimes A$;

- for every $\lambda \in \operatorname{Rep}(G)$ and $u \in C(G)_{\lambda}$, a function symbol $\mathcal{S} \rightarrow \mathcal{S}^{(1)}$ for the canonical map $A \rightarrow C(G) \otimes A$;

- for every bounded linear map $T: C(G) \rightarrow C(G)$ that maps $\mathcal{O}(G)$ to itself, a function symbol $\mathcal{S}^{(1)} \rightarrow \mathcal{S}^{(1)}$ to be interpreted as $T \otimes \mathrm{id}_{A}: C(G) \otimes A \rightarrow C(G) \otimes A$ and a function symbol $\mathcal{S}^{(0)} \rightarrow \mathcal{S}^{(0)}$ to be interpreted as $T: C(G) \rightarrow C(G)$;

- for every $\omega \in \mathcal{O}(\hat{G})$, a function symbol $\mathcal{S}^{(1)} \rightarrow \mathcal{S}$ to be interpreted as the map $\omega \otimes \operatorname{id}_{A}: C(G) \otimes A \rightarrow A$ (slice maps) and a function symbol $\mathcal{S}^{(0)} \rightarrow \mathcal{C}$ to be interpreted as $\omega: C(G) \rightarrow \mathbb{C}$;

- a function symbol $\mathcal{S} \rightarrow \mathcal{S}^{(1)}$ for the ${ }^{*}$-homomorphisms $\alpha: A \rightarrow C(G) \otimes A$ defining the action. 
3.4.2. Axioms. We now describe axioms for the class of $G$-C $C^{*}$-algebras in the language $\mathcal{L}_{G}^{\mathrm{C}^{*}}$ described above. We will use the notation introduced in Subsection 3.1 and Subsection 3.3. Particularly, we set $E_{i j}^{\lambda}:=\left(\omega_{i j}^{\lambda} \otimes \mathrm{id}\right) \circ \alpha$ for $\lambda \in \operatorname{Rep}(G)$ and $1 \leq i, j \leq d_{\lambda}$, and $E^{\lambda}:=\sum_{i=1}^{d_{\lambda}} E_{i i}^{\lambda}$. We also set $P^{\lambda} \otimes \mathrm{id}:=\sum_{i=1}^{d_{\lambda}} P_{i i}^{\lambda} \otimes \mathrm{id}$ and $P^{\lambda} \otimes E^{\lambda}:=$ $\left(P^{\lambda} \otimes \mathrm{id}\right) \circ E^{\lambda}$. The axioms are designed to guarantee the following:

(1) the interpretations of the sort $\mathcal{S}, \mathcal{S}^{(0)}$, and $\mathcal{S}^{(1)}$ are $\mathrm{C}^{*}$-algebras;

(2) the interpretation of the symbol for $\alpha$ is an isometric *-homomorphism;

(3) the sort $\mathcal{C}$ is interpreted as the complex numbers, and its domains are interpreted correctly;

(4) the interpretation of the domains $D_{n}^{\lambda, \mathcal{S}}$ is the ball of radius $n$ of the range of $E^{\lambda}$ centered at the origin (see also [12, Example 2.2.1]);

(5) the interpretation of the domain $D_{n}^{\lambda, \mathcal{S}}$ is the ball of radius $n$ of the range of $P^{\lambda} \otimes E^{\lambda}$ centered at the origin;

(6) the interpretation of the sort $\mathcal{S}^{(0)}$ is isomorphic to $C(G)$ as a $\mathrm{C}^{*}$-algebra;

(7) the interpretation of the sort $\mathcal{S}^{(1)}$ is isomorphic to $C(G) \otimes A$ as a $C(G)$-bimodule;

(8) the norm on $\mathcal{S}^{(1)}$ is the minimal (injective) tensor product norm (see Lemma 3.4);

(9) the function symbols for $T: C(G) \rightarrow C(G)$ and $T \otimes \mathrm{id}_{A}: C(G) \otimes A \rightarrow C(G) \otimes A$, where $T$ is a bounded linear map sending $\mathcal{O}(G)$ to itself, are interpreted correctly;

(10) the function symbols for the slice maps $\omega_{i j}^{\lambda} \otimes \mathrm{id}_{A}: C(G) \otimes A \rightarrow A$ are interpreted correctly;

(11) the interpretations of the symbols for $E_{i j}^{\lambda}$ satisfy

$$
E_{i j}^{\lambda} E_{s n}^{\mu}=\delta_{\lambda \mu} \delta_{i s} \delta_{j n}
$$

for $\lambda, \mu \in \operatorname{Irr}(G), 1 \leq i, j \leq d_{\lambda}$, and $1 \leq s, n \leq d_{\mu}$;

(12) the interpretations of the symbols for $E^{\lambda}$ satisfy $E^{\lambda}=E^{\lambda_{1}}+\cdots+E^{\lambda_{n}}$ whenever $\lambda \in \operatorname{Rep}(G)$ is the direct sum of irreducible unitary representations $\lambda_{1} \oplus \cdots \oplus \lambda_{n}$, and the fixed orthonormal basis on $\mathcal{H}_{\lambda}=\mathcal{H}^{\lambda_{1}} \oplus \cdots \oplus \mathcal{H}^{\lambda_{n}}$ is obtained from the fixed orthonormal bases of $\mathcal{H}^{\lambda_{1}}, \ldots, \mathcal{H}^{\lambda_{n}}$;

(13) the interpretations of the symbols for $E^{\lambda}$ and $P^{\lambda}$ satisfy

$$
\left(P_{i j}^{\lambda} \otimes \mathrm{id}\right) \circ \alpha=\alpha \circ E_{i j}^{\lambda}
$$

for every $\lambda \in \operatorname{Irr}(G)$ and $1 \leq i, j \leq d_{\lambda}$ (see part (1) of Lemma 3.5);

(14) for every $\lambda \in \operatorname{Irr}(G)$, we have

$$
1 \otimes E^{\lambda}=\sum_{1 \leq s, t \leq d_{\lambda}}\left(\left(u_{s t}^{\lambda}\right)^{*} \otimes 1\right)\left(\alpha \circ E_{s t}^{\lambda}\right) .
$$

Next, we show that the axioms (1)-(14) indeed axiomatize the class of $G$-C ${ }^{*}$-algebras.

Proposition 3.6. If $G$ is a compact quantum group, then an $\mathcal{L}_{G}^{C^{*}}$-structure satisfies (1)-(14) above if and only if it is given by a $G$ - $C^{*}$-algebra.

Proof. The discussion in Subsection 3.3 shows that any $G$-C $\mathrm{C}^{*}$-algebra $(A, \alpha)$, when regarded as an $\mathcal{L}_{G}^{\mathrm{C}^{*}}$-structure, satisfies the axioms (1)-(14) above. We prove the converse, by showing that (13) implies that the action condition from Definition 3.3 holds, and that (14) implies that the density condition from Definition 3.3 holds.

For every $\lambda \in \operatorname{Irr}(G)$ and $1 \leq i, j \leq d_{\lambda}$, in view of the axioms from (13) we have

$$
\begin{aligned}
\left(\omega_{i j}^{\lambda} \otimes \mathrm{id} \otimes \mathrm{id}\right) \circ(\mathrm{id} \otimes \alpha) \circ \alpha & =(\mathrm{id} \circ \alpha) \circ\left(\omega_{i j}^{\lambda} \otimes \mathrm{id} \otimes \mathrm{id}\right) \circ \alpha \\
& =(\mathrm{id} \circ \alpha) \circ\left(P_{i j}^{\lambda} \circ \mathrm{id}\right) \circ \alpha \\
& =\left(\omega_{i j}^{\lambda} \otimes \mathrm{id} \otimes \mathrm{id}\right) \circ(\Delta \otimes \mathrm{id}) \circ \alpha .
\end{aligned}
$$

Since this is true for every $\lambda \in \operatorname{Irr}(G)$ and $1 \leq i, j \leq d_{\lambda}$, we conclude that (id $\left.\otimes \alpha\right) \circ \alpha=(\Delta \otimes$ id) $\circ \alpha$, which is exactly the action condition from Definition 3.3 .

For the density condition, let $\lambda \in \operatorname{Irr}(G)$ and $a \in A_{\lambda}$. Then

$$
1 \otimes a=\sum_{1 \leq s, t \leq d_{\lambda}}\left(\left(u_{s t}^{\lambda}\right)^{*} \otimes 1\right)\left(\alpha \circ E_{s t}^{\lambda}\right)(a) \in\left[(C(G) \otimes 1) \alpha\left(A_{\lambda}\right)\right] .
$$

Since this holds for every $\lambda \in \operatorname{Irr}(G)$, we have that $[(C(G) \otimes 1) \alpha(A)]=C(G) \otimes A$, as desired.

Remark 3.7. One similarly checks that an $\mathcal{L}_{G}^{\mathrm{C}^{*}}$-morphism between $G$-C $\mathrm{C}^{*}$-algebra is precisely a $G$-equivariant *-homomorphism, while an $\mathcal{L}_{G}^{\mathrm{C}^{*}}$-embedding is an injective $G$-equivariant *-homomorphism. 
It is easy to see that the axioms above are given by conditions of the form $\sigma \leq r$, where $\sigma$ is a positive primitive $\forall \exists$ - $\mathcal{L}_{G}^{\mathrm{C}^{*}}$-sentence and $r \in \mathbb{R}$. Therefore, the class of $G$-C $\mathrm{C}^{*}$-algebras is positively primitively $\forall \exists$-axiomatizable in the language $\mathcal{L}_{G}^{\mathrm{C}^{*}}$, in the sense of Definition A.3.

3.4.3. Freeness. We continue to fix a compact quantum group $G$. We recall here the notion of freeness for a $G$-C*-algebra $A$ from [10].

Definition 3.8. A $G$-C $C^{*}$-algebra $(A, \alpha)$ is free if $[(1 \otimes A) \alpha(A)]=C(G) \otimes A$.

It is proved in [10, Theorem 2.9] that such a definition recovers the usual notion of freeness in topological dynamics when $G$ is a classical compact group and $A$ is an abelian $\mathrm{C}^{*}$-algebra.

Example 3.9. The left translation action of $G$ on $C(G)$ is free. More generally, if $A$ is any $C^{*}$-algebra, then the $G$-action on $C(G) \otimes A$ given by $\Delta \otimes \mathrm{id}_{A}$ is free as well.

The following equivalent reformulation of freeness is an easy consequence of the fact that $\mathcal{O}(A)$ is a dense *-subalgebra of $A$, the fact that $\mathcal{O}(G)$ is a dense *-subalgebra of $C(G)$, and part (1) of Lemma 3.5.

Lemma 3.10. A $G$-C ${ }^{*}$-algebra $(A, \alpha)$ is free if and only if $\left[(1 \otimes \mathcal{O}(A)) \alpha\left(A_{\lambda}\right)\right]$ is equal to $C(G)_{\lambda} \otimes A$, for every $\lambda \in \operatorname{Irr}(G)$. It follows that the class of free $G$-C $C^{*}$-algebras is definable by a uniform family of positive existential $\mathcal{L}_{G}^{\mathrm{C}^{*}}$-formulas.

3.4.4. Ultraproducts. Regarding $G$-C*-algebras as structures in the language $\mathcal{L}_{G}^{\mathrm{C}^{*}}$ described above provides a natural notion of ultraproduct of $G$-C $C^{*}$-algebras, as a particular instance of the notion of ultraproduct in the logic for metric structures. Since the class of $G$-C $\mathrm{C}^{*}$-algebras is axiomatizable in the language $\mathcal{L}_{G}^{\mathrm{C}^{*}}$, it follows that the ultraproduct of $G$-C $\mathrm{C}^{*}$-algebras is a $G$-C $\mathrm{C}^{*}$-algebra.

More generally, one can consider reduced products of $G$-C $\mathrm{C}^{*}$-algebras with respect to an arbitrary filter $\mathcal{F}$ as particular instances of reduced products of metric structures as defined in [23]. Concretely, suppose that $\mathcal{F}$ is a filter on a set $I$, and let $\left(A_{i}, \alpha_{i}\right)$ be an $I$-sequence of $G$-C ${ }^{*}$-algebras. For $\lambda \in \operatorname{Rep}(G)$, let $A_{\lambda}$ be the Banach space obtained as the vector space of bounded sequences $\left(a_{i}\right) \in \prod_{i \in I}\left(A_{i}\right)_{\lambda}$ endowed with the seminorm $\left\|\left(a_{i}\right)\right\|=\lim \sup _{\mathcal{F}}\left\|a_{i}\right\|$. (This is just the reduced product $\prod_{\mathcal{F}}\left(A_{i}\right)_{\lambda}$ of the $I$-sequence of Banach spaces $\left(A_{i}\right)_{\lambda}$.) One can regard $A_{\lambda}$ as a closed subspace of $A_{\mu}$ whenever $\lambda, \mu \in \operatorname{Rep}(G)$ and $\lambda$ is contained in $\mu$. Therefore, the union $\bigcup_{\lambda \in \operatorname{Rep}(G)} A_{\lambda}$ has a natural normed vector space structure. Let $A$ denote its completion. We will write $\left[a_{i}\right]_{\mathcal{F}}$ for the element of $A$ corresponding to the bounded sequence $\left(a_{i}\right)_{i \in I}$ in $\prod_{i \in I} A_{i}$.

Multiplication and involution on $A$ are induced by pointwise operations (it is important to notice here that $\left(a_{i} b_{i}\right)$ is automatically a bounded sequence in $\prod_{i \in I}\left(A_{i}\right)_{\lambda \otimes \mu}$ and $\left(a_{i}^{*}\right)$ is a bounded sequence in $\left.\prod_{i \in I}\left(A_{i}\right)_{\bar{\lambda}}\right)$. Finally, since $C(G)_{\lambda}$ is finite-dimensional for every $\lambda \in \operatorname{Rep}(G)$, one can isometrically identify $C(G)_{\lambda} \otimes A_{\lambda}$ with the reduced product of Banach spaces $\prod_{\mathcal{F}}\left(C(G)_{\lambda} \otimes\left(A_{i}\right)_{\lambda}\right)$; see for instance [26, Lemma 7.4], where the case of ultraproducts is considered. Therefore, the assignment $\left(a_{i}\right)_{i \in I} \mapsto\left(\alpha\left(a_{i}\right)\right)_{i \in I}$, induces a function $\alpha: A_{\lambda} \rightarrow \prod_{\mathcal{F}}\left(C(G)_{\lambda} \otimes\left(A_{i}\right)_{\lambda}\right)=C(G)_{\lambda} \otimes A_{\lambda}$ for every $\lambda \in \operatorname{Rep}(G)$. The induced function $\alpha: A \rightarrow C(G) \otimes A$ is easily seen to be an action of $G$ on $A$. In the following, we will denote such an action by $\alpha_{\mathcal{F}}$.

Remark 3.11. In view of [2, Proposition 2.13], considering such an explicit construction of the reduced product shows that it coincides with the continuous part of the sequence algebra as defined in [2, Definition 2.11] when $\mathcal{F}$ is the filter of cofinite subsets of $\mathbb{N}$ and $G$ is a second countable coexact compact quantum group (which is the only case considered in [2, Definition 2.11]).

We now show that, when $G$ is a classical compact group, the reduced product of $G$-C $\mathrm{C}^{*}$-algebras as $\mathcal{L}_{G}^{\mathrm{C}^{*}}$ structures agrees with the continuous part of the sequence algebra of a $G$-C $C^{*}$-algebra considered in the $\mathrm{C}^{*}$ algebra literature; see, for example, [22].

Proposition 3.12. Let $G$ be a compact group. Suppose that $\mathcal{F}$ is a filter on an index set $I$, and let $\left\{\left(A_{i}, \alpha^{(i)}\right): i \in\right.$ $I\}$ be $G$-C $C^{*}$-algebras. Denote by $\alpha_{\mathcal{F}}$ the (not necessarily continuous) action of $G$ on $\prod_{\mathcal{F}} A_{i}$ given by pointwise application of the actions $\alpha^{(i)}$. Then the reduced ultraproduct $\prod_{\mathcal{F}}^{G} A_{i}$ agrees with the subalgebra of $\prod_{\mathcal{F}} A_{i}$ where $\alpha_{\mathcal{F}}$ is continuous.

Proof. For convenience, denote by $B$ the subalgebra of $\prod_{\mathcal{F}} A_{i}$ where $G$ acts continuously. Regard $\prod_{\mathcal{F}}^{G} A_{i}$ as a $\mathrm{C}^{*}$-subalgebra of $\prod_{\mathcal{F}} A_{i}$, in such a way that the inclusion $\prod_{\mathcal{F}}^{G} A_{i} \subseteq \prod_{\mathcal{F}} A_{i}$ is $G$-equivariant. Since the action of $G$ on $\prod_{\mathcal{F}}^{G} A_{i}$ is continuous, one has $\prod_{\mathcal{F}}^{G} A_{i} \subseteq B$. On the other hand, since the action of $G$ on $B$ is continuous, 
the ${ }^{*}$-subalgebra $\mathcal{O}(B)=\bigcup_{\lambda \in \operatorname{Rep}(G)} B_{\lambda}$ consisting of the spectral subspaces for $B$, is dense in $B$. For every $\lambda \in \operatorname{Rep}(G)$, and using the projections onto the spectral subspaces, one can see directly that

$$
B_{\lambda}=\prod_{\mathcal{F}}\left(A_{i}\right)_{\lambda} \subseteq \prod_{\mathcal{F}}^{G} A_{i}
$$

Since this holds for every $\lambda \in \operatorname{Rep}(G)$, we have $\mathcal{O}(B) \subseteq \prod_{\mathcal{F}}^{G} A_{i}$ and hence $B \subseteq \prod_{\mathcal{F}}^{G} A_{i}$.

In particular, it follows from these observations that the (positive) existential theory of a $G$-C*-algebras as defined in [22, Subsection 2.2] coincides with the (positive) existential theory of a $G$-C*algebras as an $\mathcal{L}_{G}^{\mathrm{C}^{*}}$-structure.

3.4.5. Other languages. As for the case of discrete quantum group actions, it is sometimes useful to consider $\mathrm{C}^{*}$-algebras and $G$-C $\mathrm{C}^{*}$-algebras as structures in a language that is different from the standard language for $\mathrm{C}^{*}$-algebras. These other languages allow one to capture properties that are preserved under not necessarily multiplicative maps. Several such languages are considered in [22, Section 3]. If $\mathcal{L}$ is such a language, one can define its corresponding $G$-equivariant analogue $\mathcal{L}_{G}$ as above, so that $G$-C ${ }^{*}$-algebras can also be regarded as $\mathcal{L}_{G}$-structures.

\section{Crossed Products AND REduced PROducts}

4.1. The dual of a compact quantum group. The dual $\hat{G}$ of a compact quantum group $G$ is the discrete quantum group defined as follows. By assumption, the GNS representation associated with the Haar state $h$ on $C(G)$ defines a faithful representation of $C(G)$. We denote by $L^{2}(G)$ the corresponding Hilbert space, and we identify $C(G)$ with a subalgebra of $B\left(L^{2}(G)\right)$. We let $x \mapsto|x\rangle$ be the canonical map from $C(G)$ to $L^{2}(G)$, so that $x|y\rangle=|x y\rangle$, and $\langle x, y\rangle=h\left(x^{*} y\right)$ for all $x, y \in C(G)$. For $\lambda \in \operatorname{Rep}(G)$, set $L^{2}(G)_{\lambda}=\left\{u_{\xi, \eta}^{\lambda}: \xi, \eta \in \mathcal{H}_{\lambda}\right\} \subseteq L^{2}(G)$.

Recall that $\mathcal{O}(\hat{G})$ denotes the space of linear functionals on $C(G)$ of the form $x \mapsto h(x a)$ for some $a \in \mathcal{O}(G)$. It coincides with the space of linear functionals on $C(G)$ of the form $x \mapsto h(a x)$ for some $a \in \mathcal{O}(G)$, and it is also equal to the linear span of $\omega_{i j}^{\lambda}$ for $\lambda \in \operatorname{Irr}(G)$ and $1 \leq i, j \leq d_{\lambda}$. This is an algebra with respect to convolution. The antipode map $S$ of $\mathcal{O}(G)$ defines an involution on $\mathcal{O}(\hat{G})$ by setting $\phi^{*}=\overline{\phi\left(S(x)^{*}\right)}$. For $\lambda \in \operatorname{Rep}(G)$, set

$$
c_{0}(\hat{G})_{\lambda}=\operatorname{span}\left\{\omega_{\xi, \eta}^{\lambda}: \xi, \eta \in \mathcal{H}_{\lambda}\right\},
$$

and observe that $\omega_{\xi, \eta}^{\lambda} \mapsto T_{\xi, \eta}$ is a *-homomorphism from $c_{0}(\hat{G})_{\lambda}$ onto $\mathbb{K}\left(\mathcal{H}_{\lambda}\right)$, where $T_{\xi, \eta}$ is the usual rank-one operator. Furthermore $\mathcal{O}(\hat{G})$ is isomorphic to the algebraic direct sum of $c_{0}(\hat{G})_{\lambda}$ for $\lambda \in \operatorname{Irr}(G)$. One can define an injective ${ }^{*}$-representation of $\mathcal{O}(\hat{G})$ on $L^{2}(G)$ by setting $\omega|x\rangle=\mid($ id $\left.\otimes \omega) \Delta(x)\right\rangle$ for $x \in C(G)$ and $\omega \in \mathcal{O}(\hat{G})$. We will identify $\mathcal{O}(\hat{G})$ with its image inside $B\left(L^{2}(G)\right)$, and let $C^{*}(G)=c_{0}(\hat{G})$ be the closure of $\mathcal{O}(\hat{G})$ inside $B\left(L^{2}(G)\right)$. For $\lambda \in \operatorname{Rep}(G)$, the subspace $L^{2}(G)_{\lambda}$ is invariant under $c_{0}(\hat{G})_{\lambda}$, and the inclusion $c_{0}(\hat{G})_{\lambda} \subseteq B\left(L^{2}(G)_{\lambda}\right)$ is isometric. The $\mathrm{C}^{*}$-algebra $C^{*}(G)=c_{0}(\hat{G})$ is also called the group $C^{*}$-algebra of the compact quantum group $G$.

The multiplication operation on $C(G)$ defines a nondegenerate *homomorphism $\hat{\Delta}: \mathcal{O}(\hat{G}) \rightarrow M(\mathcal{O}(\hat{G}) \odot$ $\mathcal{O}(\hat{G}))$ such that $\left(\omega_{1} \otimes 1\right) \hat{\Delta}\left(\omega_{2}\right)$ belongs to $\mathcal{O}(\hat{G}) \odot \mathcal{O}(\hat{G})$ for every $\omega_{1}, \omega_{2} \in \mathcal{O}(\hat{G})$, and

$$
\begin{array}{ll}
\left(\omega_{1} \otimes 1\right) \hat{\Delta}\left(\omega_{2}\right) & : \quad x \otimes y \mapsto\left(\omega_{1} \otimes \omega_{2}\right)(\Delta(x)(1 \otimes y)) \\
\hat{\Delta}\left(\omega_{2}\right)\left(1 \otimes \omega_{1}\right) & : \quad x \otimes y \mapsto\left(\omega_{2} \otimes \omega_{1}\right)((x \otimes 1) \Delta(y)) .
\end{array}
$$

Such a nondegenerate *-homomorphism extends to $c_{0}(\hat{G})$ and defines a *-homomorphism $\hat{\Delta}: c_{0}(\hat{G}) \rightarrow M\left(c_{0}(\hat{G}) \otimes\right.$ $\left.c_{0}(\hat{G})\right)$ which is also nondegenerate. This defines a discrete quantum group $\hat{G}$, which is the dual of $G$.

For the purpose of defining the dual of a given action, it is convenient to consider the opposite discrete group $\check{G}$ of $\hat{G}$.

Definition 4.1. Let $G$ be a compact quantum group. Following the notation of [2], we define $\check{G}$ to be the discrete group such that $c_{0}(\check{G})$ is equal to $c_{0}(\hat{G})$ as a $\mathrm{C}^{*}$-algebra, but endowed with the opposite comultiplication $\check{\Delta}(\omega)=\Sigma \circ \hat{\Delta}(\omega) \circ \Sigma$.

The Hopf *-algebra $\mathcal{O}(\check{G})$ is equal to $\mathcal{O}(\hat{G})$ but endowed with the opposite comultiplication. 
4.2. The crossed product of a compact quantum group action. We fix a compact quantum group $G$ and a $G$-C $\mathrm{C}^{*}$-algebra $(A, \alpha)$. We proceed to define the associated (reduced) crossed product.

Definition 4.2. (See [6, Definition 5.28] and [6, Proposition 5.32]). Fix a nondegenerate faithful *-representation $A \rightarrow B(\mathcal{H})$, under which we regard $\alpha(A) \subseteq C(G) \otimes A$ as a subalgebra of $B\left(L^{2}(G) \otimes \mathcal{H}\right)$ and similarly for $c_{0}(\hat{G}) \otimes 1$. The reduced crossed product $G \ltimes_{\alpha, \mathrm{r}} A$ is defined as

$$
\left[\left(c_{0}(\hat{G}) \otimes 1\right) \alpha(A)\right] \subseteq B\left(L^{2}(G) \otimes \mathcal{H}\right) .
$$

The crossed product is canonically endowed with an action $\check{\alpha}$ of $\check{G}$, called the dual action, which is defined by setting

$$
\check{\alpha}((\omega \otimes 1) \alpha(a))=(\check{\Delta}(\omega) \otimes 1)(1 \otimes \alpha(a)) \in M\left(c_{0}(\check{G}) \otimes G \ltimes_{\alpha} A\right) \subseteq B\left(L^{2}(G) \otimes L^{2}(G) \otimes \mathcal{H}\right) .
$$

We will regard $G \ltimes_{\alpha, \mathrm{r}} A$ as a $\check{G}$-C $\mathrm{C}^{*}$-algebra endowed with such an action of $\check{G}$. Given $\omega \in c_{0}(\check{G})$ and $a \in A$, we denote by $\omega \ltimes a$ the element $(\omega \otimes 1) \alpha(a)$ of $G \ltimes_{\alpha, \mathrm{r}} A$. It is shown in [6, Theorem 5.31] that the reduced crossed product $G \ltimes_{\alpha, \mathrm{r}} A$ as defined above coincides with the full crossed product in the sense of [6, Definition 5.27], and therefore can also be denoted by $G \ltimes_{\alpha} A$. It follows from the universal property of the full crossed products that, if $(B, \beta)$ and $(C, \gamma)$ are $G$-C ${ }^{*}$-algebras, then a $G$-equivariant ${ }^{*}$-homomorphism $\phi: B \rightarrow C$ induces a $\check{G}$-equivariant *-homomorphism $G \ltimes \phi: G \ltimes_{\beta, \mathrm{r}} B \rightarrow G \ltimes_{\gamma, \mathrm{r}} C$ by setting $(G \ltimes \phi)(\omega \ltimes a)=\omega \ltimes \phi(a)$. Furthermore, if $\phi$ is nondegenerate, then $G \ltimes \phi$ is nondegenerate [47, Theorem 9.4.8]. Recall that a completely positive order zero map $\phi: A \rightarrow B$ between $\mathrm{C}^{*}$-algebra is order zero if, whenever $a, b$ are positive elements of $A$ satisfying $a b=b a=0$, then $\phi(a) \phi(b)=\phi(b) \phi(a)=0$ [50, Definition 2.3]. One can then easily deduce from this and the structure theorem for completely positive order zero maps the following lemma; see also [18, Proposition 2.3].

Lemma 4.3. Suppose that $(A, \alpha),(B, \beta),(C, \gamma)$ are $G$-C $C^{*}$-algebras. Assume that $A \subseteq B$ and $A \subseteq C$ are $G$-invariant subalgebras. Let $\theta: B \rightarrow C$ be a $G$-equivariant completely positive order zero $A$-bimodule map. Then the assignment

$$
\omega \ltimes b \mapsto \omega \ltimes \theta(b)
$$

defines a completely positive $\check{G}$-equivariant order zero $\left(G \ltimes_{\alpha} A\right)$-bimodule map $G \ltimes \theta: G \ltimes_{\beta} B \rightarrow G \ltimes_{\gamma} C$. If furthermore $\theta$ is a (nondegenerate) ${ }^{*}$-homomorphism, then $G \ltimes \theta$ is a (nondegenerate) *-homomorphism.

Suppose now that $\mathcal{F}$ is a filter on some index set $I$. For every $\ell \in I$, let $\left(A_{\ell}, \alpha_{\ell}\right)$ be a $G$-C*algebra. Fix also, for every $\ell \in I$, a nondegenerate faithful *-representation $A_{\ell} \rightarrow B\left(\mathcal{H}_{\ell}\right)$. Consider the corresponding reduced product of $G$-C $\mathrm{C}^{*}$-algebras $\prod_{\mathcal{F}}^{G} A_{\ell} \subseteq \prod_{\mathcal{F}} B\left(\mathcal{H}_{\ell}\right) \subseteq B\left(\prod_{\mathcal{F}} \mathcal{H}_{\ell}\right)$, which is a $G$-C $\mathrm{C}^{*}$-algebra endowed with the action $\alpha_{\mathcal{F}}$. Then the crossed product $G \ltimes_{\alpha_{\mathcal{F}}} \prod_{\mathcal{F}}^{G} A_{\ell}$ can be naturally represented on $L^{2}(G) \otimes \prod_{\mathcal{F}} \mathcal{H}_{\ell}$. On the other hand, one can also consider, for every $\ell \in I$, the crossed product $G \ltimes_{\alpha_{\ell}} A_{\ell} \subseteq B\left(L^{2}(G) \otimes \mathcal{H}\right)$, and then the reduced product of $\check{G}$-C $\mathrm{C}^{*}$-algebras

$$
\prod_{\mathcal{F}}^{\check{G}}\left(G \ltimes_{\alpha_{\ell}} A_{\ell}\right) \subseteq \prod_{\mathcal{F}} B\left(L^{2}(G) \otimes \mathcal{H}_{\ell}\right) \subseteq B\left(\prod_{\mathcal{F}}\left(L^{2}(G) \otimes \mathcal{H}_{\ell}\right)\right)
$$

These algebras do not coincide in general, but there is always a canonical map in one direction, as we show next.

Proposition 4.4. Let the notation be as in the discussion above. Then the assignment $\theta \ltimes\left[a^{(\ell)}\right]_{\mathcal{F}} \mapsto\left[\theta \ltimes a^{(\ell)}\right]_{\mathcal{F}}$, for $\lambda \in \operatorname{Rep}(G)$, for $\theta \in c_{0}(\check{G})_{\lambda}$, and $a \in\left(\prod_{\mathcal{F}}^{G} A_{\ell}\right)^{\lambda}$, determines an injective $\check{G}$-equivariant ${ }_{\text {-homomorphism }}$

$$
G \ltimes_{\alpha_{\mathcal{F}}} \prod_{\mathcal{F}}^{G} A_{\ell} \rightarrow \prod_{\mathcal{F}}^{\check{G}}\left(G \ltimes_{\alpha_{\ell}} A_{\ell}\right) .
$$

Proof. To simplify the notation, we drop the subscript $\mathcal{F}$ when denoting elements of a reduced product via their representative sequences. Suppose that

$$
a_{i j}=\left[a_{i j}^{(\ell)}\right] \in\left(\prod_{\mathcal{F}}^{G} A_{\ell}\right)_{\lambda}
$$

for $1 \leq i, j \leq d_{\lambda}$, and set

$$
z=\sum_{i j} \omega_{i j}^{\lambda} \ltimes\left[a_{i j}^{(\ell)}\right] \in G \ltimes_{\alpha_{\mathcal{F}}} \prod_{\mathcal{F}}^{G} A_{\ell} \subseteq B\left(L^{2}(G) \otimes \prod_{\mathcal{F}} \mathcal{H}\right) .
$$

Let $p_{\lambda} \in B\left(L^{2}(G)\right)$ be the orthogonal projection onto $L^{2}(G)_{\lambda}$. Then $z\left(p_{\lambda} \otimes 1\right)=\left(p_{\lambda} \otimes 1\right) z\left(p_{\lambda} \otimes 1\right)$ and $\|z\|=\left\|\left(p_{\lambda} \otimes 1\right) z p_{\lambda}\right\|$. 
For $\ell \in I$, set

$$
z^{(\ell)}=\sum_{i j} \omega_{i j}^{\lambda} \ltimes a_{i j}^{(\ell)} \in G \ltimes_{\alpha} A \subseteq B\left(L^{2}(G) \otimes \mathcal{H}\right),
$$

and

$$
\left[z^{(\ell)}\right] \in \prod_{\mathcal{U}}^{\breve{G}}\left(G \ltimes_{\alpha_{\ell}} A_{\ell}\right) \subseteq \prod_{\mathcal{F}} B\left(L^{2}(G) \otimes \mathcal{H}\right) \subseteq B\left(\prod_{\mathcal{F}}\left(L^{2}(G) \otimes \mathcal{H}\right)\right) .
$$

Then $\left[z^{(\ell)}\right]_{\mathcal{F}}\left(p_{\lambda} \otimes 1\right)=\left(p_{\lambda} \otimes 1\right)\left[z^{(\ell)}\right]\left(p_{\lambda} \otimes 1\right)$ and $\left\|\left[z^{(\ell)}\right]\right\|=\left\|\left(p_{\lambda} \otimes 1\right)\left[z^{(\ell)}\right]\left(p_{\lambda} \otimes 1\right)\right\|$. Since $p_{\lambda}$ is a finite-rank projection with range $L^{2}(G)_{\lambda}$, we have

$$
\left(p_{\lambda} \otimes 1\right)\left[z^{(\ell)}\right]\left(p_{\lambda} \otimes 1\right) \in\left(p_{\lambda} \otimes 1\right) B\left(\prod_{\mathcal{F}}\left(L^{2}(G) \otimes \mathcal{H}\right)\right)\left(p_{\lambda} \otimes 1\right)=B\left(L^{2}(G)_{\lambda} \otimes \prod_{\mathcal{F}} \mathcal{H}\right) .
$$

We conclude that

$$
\begin{aligned}
\left\|\left[z^{(\ell)}\right]\right\| & =\left\|\left(p_{\lambda} \otimes 1\right)\left[z^{(\ell)}\right]\left(p_{\lambda} \otimes 1\right)\right\|=\underset{\mathcal{F}}{\limsup \left\|\left(p_{\lambda} \otimes 1\right) z^{(\ell)}\left(p_{\lambda} \otimes 1\right)\right\|} \\
& =\left\|\left(p_{\lambda} \otimes 1\right) z\left(p_{\lambda} \otimes 1\right)\right\|=\|z\| .
\end{aligned}
$$

This shows that the assignment $\theta \ltimes\left[a^{(\ell)}\right] \mapsto\left[\theta \ltimes a^{(\ell)}\right]$ yields a well-defined isometric linear map

$$
\Phi: G \ltimes_{\alpha_{\mathcal{F}}} \prod_{\mathcal{F}}^{G} A_{\ell} \rightarrow \prod_{\mathcal{F}}^{\check{G}}\left(G \ltimes_{\alpha} A_{\ell}\right) .
$$

The fact that $\Phi$ is a $\check{G}$-equivariant ${ }^{*}$-homomorphism can be verified directly by means of the expression for the multiplication and involution in the crossed product, together with the definition of the dual action. This finishes the proof.

Suppose now that $\alpha$ is a continuous action of $\check{G}$ on a $\mathrm{C}^{*}$-algebra $A$. Fix a nondegenerate faithful *representation $A \rightarrow B(\mathcal{H})$. One defines the reduced crossed product $\check{G} \ltimes_{\alpha, \mathrm{r}} A$ to be

$$
\check{G} \ltimes_{\alpha, \mathrm{r}} A=[(C(G) \otimes 1) \alpha(A)] \subseteq B\left(L^{2}(G) \otimes \mathcal{H}\right),
$$

As before, we denote the element $(x \otimes 1) \alpha(a)$ of $\check{G} \ltimes_{\alpha, \mathrm{r}} A$ by $x \ltimes a$. The reduced crossed product is endowed with a canonical action of $G$ (dual action) defined by the *-homomorphism $\check{\alpha}: \check{G} \ltimes_{\alpha, \mathrm{r}} A \rightarrow C(G) \otimes\left(\check{G} \ltimes_{\alpha, \mathrm{r}} A\right)$, $x \ltimes a \mapsto(\Delta(x) \otimes 1)(1 \otimes a)$. The reduced crossed product construction is functorial, and a (nondegenerate) $\check{G}$-equivariant *-homomorphism $\phi$ induces a (nondegenerate) $G$-equivariant *-homomorphism $\check{G} \ltimes \phi$ between the reduced crossed products. This allows one to prove the analogue of Lemma 4.3 in this context. It is clear that, for $\lambda \in \operatorname{Rep}(G)$, the corresponding spectral subspace of $\check{G} \ltimes_{\alpha, \mathrm{r}} A$ is just

$$
\left(\check{G} \ltimes_{\alpha, \mathrm{r}} A\right)^{\lambda}=\operatorname{span}\left\{\omega \ltimes a: \omega \in C(G)_{\lambda}, a \in A\right\} .
$$

Lemma 4.5. Suppose that $G$ is a compact quantum group, $\mathcal{F}$ is a filter on a set $I$, and $\left(A_{\ell}, \alpha_{\ell}\right)$ is a $\check{G}$ - $\mathrm{C}^{*}$-algebra for every $\ell \in I$. The assignment of

$$
u \ltimes\left[a^{(\ell)}\right]_{\mathcal{F}} \mapsto\left[u \ltimes a^{(\ell)}\right]_{\mathcal{F}}
$$

for $\lambda \in \operatorname{Rep}(G), \theta \in C(G)_{\lambda}$, and $a \in \prod_{\mathcal{F}}^{\check{G}} A_{\ell}$ defines an injective $G$-equivariant *-homomorphism

$$
\check{G} \ltimes_{\alpha_{\mathcal{F}, \mathrm{r}}} \prod_{\mathcal{F}}^{\check{G}} A_{\ell} \rightarrow \prod_{\mathcal{F}}^{G}\left(\check{G}_{\alpha_{\ell}, \mathrm{r}} A_{\ell}\right) .
$$

Proof. This is easy to see directly, using the fact that $C(G)_{\lambda}$ is finite-dimensional for every $\lambda \in \operatorname{Rep}(G)$.

Remark 4.6. Consider the particular case of Lemma 4.5 when $\left(A_{\ell}, \alpha_{\ell}\right)$ is equal to a fixed $\breve{G}$-C*-algebra $(A, \alpha)$ for every $\ell \in I$. Then the $G$-equivariant ${ }^{*}$-isomorphism $\theta: \check{G} \ltimes_{\alpha_{\mathcal{F}, \mathrm{r}}} \prod_{\mathcal{F}}^{\check{G}} A \rightarrow \prod_{\mathcal{F}}^{G}\left(\check{G} \ltimes_{\alpha, \mathrm{r}} A\right)$ has the property that

$$
\theta \circ\left(\check{G} \ltimes \Delta_{A}\right)=\Delta_{\breve{G} \ltimes \alpha, \mathrm{r} A},
$$

where $\Delta_{A}: A \rightarrow \prod_{\mathcal{F}}^{\breve{G}} A$ and $\Delta_{\breve{G} \ltimes_{\alpha, \mathrm{r}} A}: \check{G} \ltimes_{\alpha, \mathrm{r}} A \rightarrow \prod_{\mathcal{F}}^{G}\left(\check{G} \ltimes_{\alpha, \mathrm{r}} A\right)$ are the diagonal embeddings. 
4.3. Stabilizations. We continue to fix a compact quantum group $G$. Let $(A, \alpha)$ is a $G$-C $\mathrm{C}^{*}$-algebra. Fix a nondegenerate faithful representation $A \rightarrow B(\mathcal{H})$. Let $V \in M\left(c_{0}(\hat{G}) \otimes C(G)\right) \subseteq B\left(L^{2}(G) \otimes L^{2}(G)\right)$ denote the multiplicate unitary associated with $G$, and set $X=\Sigma \circ V \circ \Sigma \in M\left(C(G) \otimes c_{0}(\hat{G})\right) \subseteq B\left(L^{2}(G) \otimes L^{2}(G)\right)$.

Definition 4.7. ([43, Section 2]). Adopt the notation from the discussion above. The stabilization of $(A, \alpha)$ is the $G$-C ${ }^{*}$-algebra $\left(\mathbb{K}_{G} \otimes A, \alpha_{\mathbb{K}}\right)$, where $\alpha_{\mathbb{K}}: \mathbb{K}_{G} \otimes A \rightarrow C(G) \otimes \mathbb{K}_{G} \otimes A$ is the ${ }^{*}$-homomorphism given by

$$
\alpha_{\mathbb{K}}(T \otimes a)=X_{12}^{*}(1 \otimes T \otimes 1) \alpha(a)_{13} X_{12} \in C(G) \otimes \mathbb{K}_{G} \otimes A \subseteq B\left(L^{2}(G) \otimes L^{2}(G) \otimes \mathcal{H}\right) .
$$

In the following, given a $G$-C $C^{*}$-algebra $(A, \alpha)$, we regard $\mathbb{K}_{G} \otimes A$ as a $G$-C ${ }^{*}$-algebra with respect to $\alpha_{\mathbb{K}}$.

When $G$ is a classical compact group, $\alpha_{\mathbb{K}}$ is just the diagonal action, where $\mathbb{K}_{G}$ is endowed with the action of $G$ by conjugation induced by the left regular representation.

The assignment $(A, \alpha) \mapsto\left(\mathbb{K}_{G} \otimes A, \alpha_{\mathbb{K}}\right)$ is functorial, in the sense that any *-homomorphism $\phi:(A, \alpha) \rightarrow$ $(B, \beta)$ induces a ${ }^{*}$-homomorphism id $\otimes \phi:\left(\mathbb{K}_{G} \otimes A, \alpha_{\mathbb{K}}\right) \rightarrow\left(\mathbb{K}_{G} \otimes B, \beta_{\mathbb{K}}\right)$.

Proposition 4.8. Let $\mathcal{F}$ be a filter on a set $I$, and let $\left(A_{\ell}, \alpha^{(\ell)}\right)_{\ell \in I}$ be $G$ - $C^{*}$-algebras. Then the assignment $T \otimes\left[a^{(\ell)}\right]_{\mathcal{F}} \mapsto\left[T \otimes a^{(\ell)}\right]_{\mathcal{F}}$ defines a G-equivariant *-homomorphism

$$
\mathbb{K}_{G} \otimes \prod_{\mathcal{F}}^{G} A_{\ell} \rightarrow \prod_{\mathcal{F}}^{G}\left(\mathbb{K}_{G} \otimes A_{\ell}\right)
$$

Proof. Observe that tensor product $\mathbb{K}_{G} \otimes \prod_{\mathcal{F}}^{G} A_{\ell}$ coincides with the maximal tensor product. Therefore the assignment in the statement gives a well-defined *-homomorphism $\psi: \mathbb{K}_{G} \otimes \prod_{\mathcal{F}}^{G} A_{\ell} \rightarrow \prod_{\mathcal{F}}^{G}\left(\mathbb{K}_{G} \otimes A_{\ell}\right)$ in view of the universal property of the maximal tensor product. A straightforward computation shows that such a map is $G$-equivariant when $\mathbb{K}_{G} \otimes \prod_{\mathcal{F}}^{G} A_{\ell}$ is endowed with the stabilization of the reduced product action $\alpha_{\mathcal{F}}$, and $\prod_{\mathcal{F}}^{G}\left(\mathbb{K}_{G} \otimes A_{\ell}\right)$ is endowed with the reduced product of the stabilizations $\left(\alpha_{\mathcal{F}}^{(\ell)}\right)_{\ell \in I}$.

Denote by $1 \in C(G)$ the unit, and let $|1\rangle \in L^{2}(G)$ be the corresponding vector, and $|1\rangle\langle 1| \in \mathbb{K}_{G}$ the corresponding rank one projection.

Lemma 4.9. The $\mathrm{C}^{*}$-subalgebra $|1\rangle\langle 1| \otimes A \subseteq \mathbb{K}_{G} \otimes A$ is a $G$-invariant $\mathrm{C}^{*}$-subalgebra of $\left(\mathbb{K}_{G} \otimes A, \alpha_{\mathbb{K}}\right)$, and the injective ${ }^{*}$-homomorphism $a \mapsto|1\rangle\langle 1| \otimes a$ is $G$-equivariant.

Proof. The invariance property of the Haar state $h$ can be written as $h(y) 1=\sum_{i} h\left(y_{0, i}\right) y_{1, i}=\sum_{i} h\left(y_{1, i}\right) y_{0, i}$ where $\Delta(y)=\sum_{i} y_{0, i} \otimes y_{1, i}$. We claim that $(1 \otimes|1\rangle\langle 1|) X=1 \otimes|1\rangle\langle 1|$. Indeed for every $x, y \in C(G)$ we have that

$$
\begin{aligned}
(1 \otimes|1\rangle\langle 1|) X|x \otimes y\rangle & =(1 \otimes|1\rangle\langle 1|) \Sigma V|y \otimes x\rangle=(1 \otimes|1\rangle\langle 1|) \Sigma|\Delta(y)(1 \otimes x)\rangle \\
& =(1 \otimes|1\rangle\langle 1|)\left|\Delta^{\mathrm{op}}(y)(x \otimes 1)\right\rangle=\sum_{i}(1 \otimes|1\rangle\langle 1|)\left|y_{1, i} x \otimes y_{0, i}\right\rangle \\
& =\sum_{i} h\left(y_{0, i}\right)\left|y_{1, i} x \otimes 1\right\rangle=h(y)|x \otimes 1\rangle=(1 \otimes|1\rangle\langle 1|)|x \otimes y\rangle .
\end{aligned}
$$

Henceforth

$$
\begin{aligned}
\alpha_{\mathbb{K}}(|1\rangle\langle 1| \otimes a) & =X_{12}(1 \otimes|1\rangle\langle 1| \otimes 1) \alpha(a)_{13} X_{12}=X_{12}(1 \otimes|1\rangle\langle 1| \otimes 1) \alpha(a)_{13}(1 \otimes|1\rangle\langle 1| \otimes 1) X_{12} \\
& =(1 \otimes|1\rangle\langle 1| \otimes 1) \alpha(a)_{13}(1 \otimes|1\rangle\langle 1| \otimes 1)=(1 \otimes|1\rangle\langle 1| \otimes 1) \alpha(a)_{13} .
\end{aligned}
$$

This concludes the proof.

In the following lemma, we consider the ordered selfadjoint operator space language $\mathcal{L}^{\text {osos }}$ as introduced in [22, Subsection 3.1], as well as its $A$-bimodule version $\mathcal{L}^{\text {osos, } A-A}$ as introduced in [22, Subsection 3.5]. Recall that the language $\mathcal{L}^{\operatorname{osos}, A-A}$ does not have a distinguished relation symbol for the metric. Instead, for every finite subset $F$ of $A$, the language $\mathcal{L}^{\text {osos, } A-A}$ contains a distinguished pseudometric symbol $d_{F}$, to be interpreted in $\mathbb{K}_{G} \otimes A$ as the pseudometric

$$
d_{F}^{A}(x, y)=\sup \{\|(1 \otimes a)(x-y)\|,\|(x-y)(1 \otimes a)\|: a \in F\} .
$$

Given a compact or discrete quantum group $G$, one can add symbols for the $G$-action to obtain languages $\mathcal{L}_{G}^{\text {osos }}$ and $\mathcal{L}_{G}^{\text {osos, } A-A}$. If $A$ is a $G$-C ${ }^{*}$-algebra, then $\mathbb{K}_{G} \otimes A$ can be regarded as structure in the language of $\mathcal{L}_{G}^{\mathrm{C}^{*}, \mathbb{K}_{G} \otimes A-\mathbb{K}_{G} \otimes A}$.

Fix an increasing approximate unit $\left(u_{j}\right)_{j \in J}$ for $A$ contained in $A^{\alpha}$. Suppose now that $B$ is a $G$-C $\mathrm{C}^{*}$-algebra containing $A$ as a nondegenerate $G$-C $C^{*}$-subalgebra. In particular the approximate unit $\left(u_{j}\right)_{j \in J}$ for $A$ is also 
an approximate unit for $B$. The notion of positively quantifier-free definable substructure in a language $\mathcal{L}$ is recalled in Definition A.14.

Lemma 4.10. Adopt the notation and assumptions from the discussion above. The $G$-C ${ }^{*}$-subalgebra $|1\rangle\langle 1| \otimes B$ of $\mathbb{K}_{G} \otimes B$ is a positively quantifier-free $\mathcal{L}_{G}^{\text {osos, } \mathbb{K}_{G} \otimes A-\mathbb{K}_{G} \otimes A}$-definable substructure relative to the class of $G$-C*algebras of the form $\mathbb{K}_{G} \otimes B$.

Proof. We have already observed that $|1\rangle\langle 1| \otimes B$ is indeed a $G$-C ${ }^{*}$-subalgebra of $\mathbb{K}_{G} \otimes B$. The fact that it is positively quantifier-free $\mathcal{L}_{G}^{\text {osos, } \mathbb{K}_{G} \otimes A \text { - } \mathbb{K}_{G} \otimes A}$-definable is witnessed by the formulas $\varphi_{j, F}(x)$ defined by

$$
\sup _{a \in F}\left\|a\left(\left(|1\rangle\langle 1| \otimes u_{j}\right) x\left(|1\rangle\langle 1| \otimes u_{i}\right)-x\right)\right\|
$$

where $\left(u_{j}\right)_{j \in J}$ is the fixed approximate unit for $A^{\alpha}$, and $F$ ranges among the finite subsets of $\mathbb{K}_{G} \otimes A$.

In the statement of Lemma 4.10, it is important that the $G$-C $\mathrm{C}^{*}$-algebra $\mathbb{K}_{G} \otimes B$ is regarded as a structure in the language $\mathcal{L}_{G}^{\text {osos, } \mathbb{K}_{G} \otimes A-\mathbb{K}_{G} \otimes A}$, rather than a structure in the language $\mathcal{L}_{G}^{\text {osos }}$.

Remark 4.11. A similar discussion as above can be done when $(A, \alpha)$ is a $\hat{G}$-C*-algebra. Fix a nondegenerate faithful representation $A \rightarrow B(\mathcal{H})$. Set $\hat{X}=V^{*} \in M\left(c_{0}(\hat{G}) \otimes C(G)\right)$. We define the stabilization of $(A, \alpha)$ as the $\hat{G}$-C $\mathrm{C}^{*}$-algebra $\left(\mathbb{K}_{G} \otimes A, \alpha_{\mathbb{K}}\right)$, where the action $\alpha_{\mathbb{K}}$ is defined by

$$
\alpha_{\mathbb{K}}(T \otimes a)=\hat{X}_{12}^{*}(1 \otimes T \otimes 1) \alpha(a)_{13} \hat{X}_{12} \in c_{0}(\hat{G}) \in \mathbb{K}_{G} \otimes A \subseteq B\left(L^{2}(G) \otimes L^{2}(G) \otimes \mathcal{H}\right) .
$$

\section{Existential Embeddings AND the Rokhlin PROperty}

5.1. Existential embeddings. Let $G$ be either a compact or discrete quantum group. Considering $G$ $\mathrm{C}^{*}$-algebras as $\mathcal{L}_{G}^{\mathrm{C}^{*}}$-structures gives the notion of positively $\mathcal{L}_{G}^{\mathrm{C}^{*}}$-existential ${ }^{*}$-homomorphism between $G$-C*algebras; see Definition A.10. When the algebras are separable, and the group is second countable and either compact and coexact or discrete and exact (which is the only case considered in [2]), a $G$-equivariant homomorphism is positively $\mathcal{L}_{G}^{\mathrm{C}^{*}}$-existential if and only if it is sequentially split in the sense of [2, Definition 3.1]; see A.11. In this section, we show that the results from [2], phrased in terms of positive existential embeddings, can be obtained without any assumptions on the algebras or the group.

Positive existential *-homomorphisms are preserved by functors under general assumptions. If $G$ is a compact or discrete quantum group, we regard $G$-C $C^{*}$-algebras as the objects of a category with $G$-equivariant *-homomorphisms as morphisms. In the following proposition, we denote by $\Delta_{A}: A \rightarrow \prod_{\mathcal{U}}^{G} A$ the canonical diagonal $\mathcal{L}_{G}^{\mathrm{C}^{*}}$-embedding of a $G$-C $\mathrm{C}^{*}$-algebra into the corresponding ultrapower.

Proposition 5.1. Let $G_{0}$ and $G_{1}$ be either compact or discrete quantum groups, let $F$ be a functor from the category of $G_{0}-C^{*}$-algebras to the category of $G_{1}-C^{*}$-algebras. Assume that for any index set $I$, for any countably incomplete ultrafilter $\mathcal{U}$ over $I$, and for any $G_{0}-C^{*}$-algebra $A$, there exists a $G_{1}$-equivariant ${ }^{*}$-homomorphism $\theta: F\left(\prod_{\mathcal{U}}^{G_{0}} A\right) \rightarrow \prod_{\mathcal{U}}^{G_{1}} F(A)$ such that $\theta \circ F\left(\Delta_{A}\right)=\Delta_{F(A)}$. Then $F$ maps $\mathcal{L}_{G_{0}}^{C^{*}}$-existential ${ }^{*}$-homomorphisms to $\mathcal{L}_{G_{1}}^{C^{*}}$-existential *-homomorphisms.

Proof. This is immediate using the semantic characterization of positively existential morphisms from Proposition A.11.

Let $\phi:(A, \alpha) \rightarrow(B, \beta)$ be an injective nondegenerate *-homomorphism. We identify $A$ with a $G$-invariant subalgebra of $B$ via $\phi$. Suppose that $A_{0}$ is a $G$-C ${ }^{*}$-subalgebra of $A$. Both $A$ and $B$ have a natural $A_{0}$-bimodule structure, and hence can be regarded as structures in the language $\mathcal{L}_{G}^{\mathrm{C}^{*}, A_{0}-A_{0}}[22$, Subsection 3.5]. Recall that this is obtained from the language of $G$-C $C^{*}$-algebras by adding function symbols for the $A_{0}$-bimodule structure, and replacing the distinguished relation symbol for the metric with pseudometric function symbols $d_{F}$ where $F$ ranges among the finite subsets of $A_{0}$. Then $d_{F}$ is interpreted in $B$ as the pseudometric

$$
d_{F}(x, y)=\sup \{\|a(x-y)\|,\|(x-y) a\|: a \in F\} .
$$

Proposition 5.2. Let $G$ be a compact or discrete quantum group, let $(A, \alpha)$ and $(B, \beta)$ be $G$ - $C^{*}$-algebras, and let $\phi: A \rightarrow B$ be a nondegenerate injective $G$-equivariant ${ }^{*}$-homomorphism. Fix a $G$ - $C^{*}$-subalgebra $A_{0}$ of $A$ containing an approximate unit for $A$. Then $\phi$ is $\mathcal{L}_{G}^{C^{*}}$-existential if and only if it is $\mathcal{L}_{G}^{C^{*}, A_{0}-A_{0}}$-existential.

Proof. As remarked above, one can assume that $\phi: A \rightarrow B$ is the inclusion map. The forward implication is obvious. The converse implication is easily shown using an increasing approximate unit for $A$ contained in $A_{0}$, which is also an approximate unit for $B$ since the inclusion $A \subseteq B$ is nondegenerate by assumption. 
A reason to consider the notion of positive $\mathcal{L}_{G}^{\mathrm{C}^{*}}$-existential *-homomorphism is that it allows one to conclude that several properties pass from the target algebra to the domain algebra; see also [1]. The following proposition is just a special instance of Proposition A.12.

Proposition 5.3. Let $G$ be a compact or discrete quantum group. Suppose that $\mathcal{C}$ is a class of $G$ - $C^{*}$-algebras that is definable by a uniform family of positive existential $\mathcal{L}_{G}^{C^{*}}$-formulas. Suppose that $(A, \alpha)$ and $(B, \beta)$ are $G$ - $C^{*}$-algebras, and $\phi:(A, \alpha) \rightarrow(B, \beta)$ is an $\mathcal{L}_{G}^{C^{*}}$-existential ${ }^{*}$-homomorphism. If $(B, \beta)$ belongs to $\mathcal{C}$, then $(A, \alpha)$ belongs to $\mathcal{C}$.

The following result is established in [2, Proposition 3.3] when $G$ is compact and coexact or discrete and exact, and second countable. Here, we remove these assumptions.

Proposition 5.4. Let $G$ be a compact or discrete quantum group, and let $(A, \alpha)$ and $(B, \beta)$ be $G$ - $C^{*}$-algebras. Suppose that $\phi: A \rightarrow B$ is a nondegenerate $G$-equivariant ${ }^{*}$-homomorphism. If $\phi$ is positively $\mathcal{L}_{G}^{C^{*}}$-existential, then $G \ltimes \phi:\left(G \ltimes_{\alpha, \mathrm{r}} A, \check{\alpha}\right) \rightarrow\left(G \ltimes_{\beta, \mathrm{r}} B, \check{\beta}\right)$ is positively $\mathcal{L}_{\breve{G}}^{C^{*}}$-existential.

Proof. When $G$ is compact, this is a consequence of Proposition 5.1 and Proposition 4.4. When $G$ is discrete, this follows from Lemma 4.5 and Remark 4.6, together with the semantic characterization of positively existential embeddings from Proposition A.11.

The following result is established in [2, Proposition 3.6, Proposition 3.7, Proposition 3.8] when $G$ is coexact, and second countable. Here, we remove these assumptions, and provide a simpler proof.

Proposition 5.5. Let $G$ be a compact quantum group, let $(A, \alpha)$ and $(B, \beta)$ be $G$ - $C^{*}$-algebras, and let $\phi: A \rightarrow B$ be a nondegenerate $G$-equivariant *-homomorphism.

(1) If $\phi$ is positively $\mathcal{L}_{G}^{C^{*}}$-existential, then $\left.\phi\right|_{A^{\alpha}}: A^{\alpha} \rightarrow B^{\beta}$ is positively $\mathcal{L}^{C^{*}}$-existential.

(2) $\phi:(A, \alpha) \rightarrow(B, \beta)$ is positively $\mathcal{L}_{G}^{C^{*}}$-existential if and only if id $\otimes \phi:\left(\mathbb{K}_{G} \otimes A, \alpha_{\mathbb{K}}\right) \rightarrow\left(\mathbb{K}_{G} \otimes B, \beta_{\mathbb{K}}\right)$ is positively $\mathcal{L}_{G}^{C^{*}}$-existential.

(3) $\phi$ is positively $\mathcal{L}_{G}^{C^{*}}$-existential if and only if $G \ltimes \phi:\left(G \ltimes_{\alpha, \mathrm{r}} A, \check{\alpha}\right) \rightarrow\left(G \ltimes{ }_{\beta, \mathrm{r}} B, \check{\beta}\right)$ is positively $\mathcal{L}_{\tilde{G}}^{C^{*}}$ existential.

Proof. (1): This is an immediate consequence of Proposition A.15, after observing that the fixed point algebra of a $G$-C $\mathrm{C}^{*}$-algebra is an $\mathcal{L}_{G}^{\mathrm{C}^{*}}$-definable $G$-C $\mathrm{C}^{*}$-subalgebra.

(2): We can assume that $A \subseteq B$ and $\phi: A \rightarrow B$ is the inclusion map. The forward implication is a consequence of Proposition 5.3 and Proposition 4.8. For the converse, observe that we can identify $A$ with the $G$-C ${ }^{*}$-subalgebra $|1\rangle\langle 1| \otimes A$ of $\mathbb{K}_{G} \otimes A$. A similar observation applies to $B$, so we identify id $\otimes \phi$ with the inclusion map $\mathbb{K}_{G} \otimes A \subseteq \mathbb{K}_{G} \otimes B$, and $\phi$ with the restriction of id $\otimes \phi$ to $|1\rangle\langle 1| \otimes A$. We can regard $\mathbb{K}_{G} \otimes A$ and $\mathbb{K}_{G} \otimes B$ as $\mathbb{K}_{G} \otimes A$-bimodules, and hence as structures in the language $\mathcal{L}_{G}^{\mathrm{C}^{*}, \mathbb{K}_{G} \otimes A-\mathbb{K}_{G} \otimes A}$. Observe that id $\otimes \phi$ is nondegenerate. It follows from Proposition 5.2 that id $\otimes \phi$ is positively $\mathcal{L}_{G}^{\mathrm{C}}$, $\mathbb{K}_{G} \otimes A-\mathbb{K}_{G} \otimes A$-existential. Furthermore, by Lemma 4.10, $|1\rangle\langle 1| \otimes A \subseteq \mathbb{K}_{G} \otimes A$ and $|1\rangle\langle 1| \otimes B \subseteq \mathbb{K}_{G} \otimes B$ are positively quantifierfree $\mathcal{L}_{G}^{\mathrm{C}^{*}, \mathbb{K}_{G} \otimes A-\mathbb{K}_{G} \otimes A}$-definable. Therefore $\phi$ is positively $\mathcal{L}_{G}^{\mathrm{C}^{*}, \mathbb{K}_{G} \otimes A-\mathbb{K}_{G} \otimes A}$-existential by Proposition A.15. In particular, $\phi$ is positively $\mathcal{L}_{G}^{\mathrm{C}^{*}, A-A}$-existential. Since $\phi$ is nondegenerate, a further application of Proposition 5.2 shows that $\phi$ is positively $\mathcal{L}_{G}^{\mathrm{C}^{*}}$-existential. This concludes the proof.

(3): The forward implication is a consequence of Proposition 5.4. The converse implication follows from the other implication and Item (2) above, in view of the Baaj-Skandalis-Takesaki-Takai duality for compact and discrete quantum groups; see [2, Theorem 1.20], [47, Chapter 9], [6, Theorem 5.33].

5.2. The Rokhlin property. A generalization of the Rokhlin property for actions of classical compact groups on separable $\mathrm{C}^{*}$-algebras has been considered in [2, Section 4] for coexact second countable compact quantum groups. Here, we remove all separability and coexactness assumptions. We fix a compact quantum group $G$ throughout the rest of this section.

Definition 5.6. A $G$-C $C^{*}$-algebra $(A, \alpha)$ is said to have the (spatial) Rokhlin property if the map $\alpha:(A, \alpha) \rightarrow$ $\left(C(G) \otimes A, \Delta \otimes \operatorname{id}_{A}\right)$ is positively $\mathcal{L}_{G}^{\mathrm{C}^{*}}$-existential.

It follows from [2, Lemma 1.24] and Proposition A.11 that Definition 5.6 agrees with [2, Definition 4.1] in the particular case when $G$ is coexact and second countable, and $A$ is separable (which is the only case considered in [2, Definition 4.1]).

The following result generalizes [2, Proposition 4.5], with a simple and conceptual proof. 
Proposition 5.7. Suppose that $G$ is a compact quantum group, and $(A, \alpha)$ is a $G$ - $C^{*}$-algebra with the Rokhlin property. Then $(A, \alpha)$ is free.

Proof. Observe that $\left(C(G) \otimes A, \Delta \otimes \mathrm{id}_{A}\right)$ is a free $G$-C $C^{*}$-algebra. If $(A, \alpha)$ has the Rokhlin property, then $(A, \alpha)$ is free in view of this observation and Proposition 5.3.

The main result of [2] asserts that, whenever $G$ is a coexact second countable compact quantum groups and $(A, \alpha)$ is a separable $G$-C ${ }^{*}$-algebra, then several properties of $A$ are preserved under taking crossed products or passing to the fixed point algebra. One can deduce the natural generalization of such a statement to arbitrary compact quantum groups from the properties of positively existential embeddings established above.

Theorem 5.8. Let $(A, \alpha)$ be a $G$ - $C^{*}$-algebra with the Rokhlin property. Then the canonical nondegenerate inclusion $A^{\alpha} \hookrightarrow A$ is positively $\mathcal{L}^{C^{*}}$-existential, and the canonical $\breve{G}$-equivariant nondegenerate inclusion $G \ltimes_{\alpha}$ $A \hookrightarrow \mathbb{K}_{\breve{G}} \otimes A$ is positively $\mathcal{L}_{\breve{G}}^{C^{*}}$-existential. Here $\mathbb{K}_{\breve{G}} \otimes A$ is regarded as a $\check{G}^{-} C^{*}$-algebra endowed with the stabilization of the trivial action of $\check{G}$ on $A$.

Proof. If $(A, \alpha)$ has the Rokhlin property, then by definition $\alpha:(A, \alpha) \rightarrow\left(C(G) \otimes A, \Delta \otimes \mathrm{id}_{A}\right)$ is positively $\mathcal{L}_{G}^{\mathrm{C}^{*}}$-existential. By part (1) of Proposition 5.5, the restriction of $\alpha$ to the fixed point algebras is positively $\mathcal{L}^{\mathrm{C}^{*}}$-existential. Observing that the fixed point algebra of $\left(C(G) \otimes A, \Delta \otimes \operatorname{id}_{A}\right)$ is equal to $1 \otimes A \subseteq C(G) \otimes A$, we deduce that the embedding $A^{\alpha} \hookrightarrow 1 \otimes A$ is positively $\mathcal{L}^{\mathrm{C}^{*}}$-existential. This concludes the proof of the first assertion.

By Proposition 5.4, the positively $\mathcal{L}_{G}^{\mathrm{C} *}$-existential *-homomorphism $\alpha:(A, \alpha) \rightarrow\left(C(G) \otimes A, \Delta \otimes \mathrm{id}_{A}\right)$ induces a positively $\mathcal{L}_{\widetilde{G}}^{\mathrm{C}^{*}}$-existential *-homomorphism $G \ltimes \alpha: G \ltimes_{\alpha} A \rightarrow G \ltimes_{\Delta \otimes \mathrm{id}_{A}}(C(G) \otimes A)$. A particular instance of the Baaj-Skandalis-Takesaki-Takai duality for compact quantum groups - see [2, Theorem 1.20], [47, Chapter 9], [6, Theorem 5.33] — gives that there exists a $\breve{G}$-equivariant *-isomorphism $\rho: G \ltimes_{\Delta \otimes \operatorname{id}_{A}}(C(G) \otimes A) \rightarrow \mathbb{K}_{G} \otimes A$ such that $\rho \circ(G \ltimes \alpha): G \ltimes_{\alpha} A \rightarrow \mathbb{K}_{G} \otimes A$ is the canonical inclusion. Therefore we conclude that the latter *-homomorphism is positively $\mathcal{L}_{\tilde{G}}^{\mathrm{C}^{*}}$-existential as well.

As an application, we extend several preservations results for crossed products by actions with the Rokhlin property; see [17] and [1]. When $G$ is coexact and second countable, this recovers the main result of [2], although the assertions concerning real rank and stable rank are new even in this case.

Corollary 5.9. Let $(A, \alpha)$ be a $G$ - $C^{*}$-algebra with the Rokhlin property. If $A$ satisfies any of the following properties, then so do the fixed point algebra $A^{\alpha}$ and the crossed product $G \ltimes_{\alpha} A$ :

(1) being simple;

(2) being separable, nuclear, and satisfying the UCT;

(3) being separable and $\mathcal{D}$-absorbing for a given strongly self-absorbing $C^{*}$-algebra $\mathcal{D}$ or for $\mathcal{D}=\mathbb{K}(\mathcal{H})$;

(4) being expressible as a direct limit of certain weakly semiprojective $C^{*}$-algebras (see Theorem 3.10 in [17] for the precise statement). This includes UHF-algebras (or matroid algebras), AF-algebras, AI-algebras, AT-algebras, countable inductive limits of one-dimensional NCCW-complexes, and several other classes.

(5) having nuclear dimension at most $n$;

(6) having decomposition rank at most $n$;

(7) having real rank at most $n$;

(8) having stable rank at most $n$.

Proof. In view of Theorem 5.8, the canonical inclusions $A^{\alpha} \hookrightarrow A$ and $G \ltimes_{\alpha} A \hookrightarrow \mathbb{K}_{G} \otimes A$ are positively $\mathcal{L}_{G}^{\mathrm{C}^{*}}$-existential. Items $(1),(3),(4),(5),(8),(9)$ can be obtained by observing that the corresponding properties are definable by a uniform family of $\mathcal{L}_{G}^{\mathrm{C}^{*}}$-formulas as shown in [12, Theorem 2.5.1, Theorem 2.5.2] and [22, Proposition 4.3]; see also [22, Remark 3.2]. The items (2), (6), (7) can alternatively be obtained by applying the fact that the corresponding properties are definable by a uniform family of $\mathcal{L}_{G}^{\mathrm{C} \text {, nuc }}$-formulas as shown in [12, Section 5]; see also [22, Remark 3.5]. The language $\mathcal{L}_{G}^{\mathrm{C}^{*} \text {,nuc }}$ is the nuclear language for $\mathrm{C}^{*}$-algebras introduced in $[22$, Subsection 3.3].

Finally, the K-theory formula for fixed point algebras of Rokhlin actions of finite groups from [29] generalizes to the setting of Rokhlin actions of compact quantum groups. This has been shown in [2, Theorem 5.11] for separable coexact compact quantum groups, but the proof applies equally well in general.

Theorem 5.10. Let $G$ be a compact quantum group, and $(A, \alpha)$ be a $G$ - $C^{*}$-algebra. If $(A, \alpha)$ has the Rokhlin property, then the canonical inclusion $A^{\alpha} \hookrightarrow A$ induces an injective morphism $K_{*}\left(A^{\alpha}\right) \hookrightarrow K_{*}(A)$ in $K$-theory, 
whose range is

$$
\left\{x \in K_{*}(A): K_{*}(\alpha)(x)=K_{*}\left(\iota_{A}\right)(x)\right\},
$$

where $\iota_{A}: A \rightarrow C(G) \otimes A, a \mapsto 1 \otimes a$ is the trivial action of $G$ on $A$.

In fact, under the assumptions of Theorem 5.10, one can conclude that the inclusion of $K_{0}\left(A^{\alpha}\right)$ into $K_{0}(A)$ is positively existential. Here we regard $K_{0}$-group as structures in the language of dimension groups $(G,+, u)$ endowed with domains of quantifications to be interpreted as the subsets $\{x \in G:-n u \leq x \leq n u\}$ for $n \in \mathbb{N}$. This corresponds to the notion of ultrapower of dimension groups considered in [46].

5.3. Rigidity. Suppose that $G$ is a compact quantum group, and $(B, \beta)$ is a $G$-C $\mathrm{C}^{*}$-algebra. Let $\tilde{B}$ be the minimal unitization of $B$. Then the $G$-action $\beta$ has a unique extension to a $G$-action $\beta$ on $\tilde{B}$. It is easy to see that if a ${ }^{*}$-homomorphism $\phi: A \rightarrow B$ is positively $\mathcal{L}_{G}^{\mathrm{C}^{*}}$-existential, then its unique extension to a unital *-homomorphism $\phi: \tilde{A} \rightarrow \tilde{B}$ is positively $\mathcal{L}_{G}^{\mathrm{C}^{*}, 1}$-existential, where $\mathcal{L}_{G}^{\mathrm{C}^{*}, 1}$ is the language obtained from $\mathcal{L}_{G}^{\mathrm{C} *}$ by adding a constant symbols for the multiplicative unit. The following definition is introduced in the setting of compact quantum group actions in [2, Definition 5.1].

Definition 5.11. Let $G$ be a compact quantum group, $(A, \alpha),(B, \beta)$ be two $G$-C $C^{*}$-algebras, and $\phi_{1}, \phi_{2}: A \rightarrow$ $B$ be $G$-equivariant *-homomorphisms. Then $\phi_{1}, \phi_{2}$ are approximately $G$-unitarily equivalent, in formulas $\phi_{1} \approx_{\mathrm{u}, G} \phi_{2}$, if there exists a net $\left(v_{i}\right)$ of unitary elements of the fixed point algebra $\tilde{B}^{\beta}$ such that $\phi_{2}$ is the limit of $\operatorname{Ad}\left(v_{i}\right) \circ \phi_{1}$ is the topology of pointwise norm convergence.

When $B$ is separable, one can replace nets with sequences in Definition 5.11. In the case when $G$ is the trivial group, Definition 5.11 recovers the usual notion of approximate unitary equivalence $\phi_{1} \approx_{\mathrm{u}} \phi_{2}$ for the *-homomorphisms $\phi_{1}, \phi_{2}$.

A rigidity result for Rokhlin actions of coexact second countable compact quantum groups, generalizing results for finite and compact from [20, 22, 29, 42] and for finite quantum groups from [33], has been obtained in [2, Theorem 5.10]. In the rest of this section, we observe here that such a result holds for arbitrary (not necessarily coexact) second countable compact quantum groups.

Theorem 5.12. Let $G$ be a second countable compact quantum group, let $A$ be a separable $C^{*}$-algebra, and let $\alpha^{(0)}, \alpha^{(1)}$ be G-actions on $A$. If $\left(A, \alpha^{(0)}\right)$ and $\left(A, \alpha^{(1)}\right)$ have the Rokhlin property, then $\alpha^{(0)} \approx_{\mathrm{u}} \alpha^{(1)}$ if and only if $\alpha^{(0)} \approx_{\mathrm{u}, G} \alpha^{(1)}$.

One can deduce from Theorem 5.12 the following corollary, similarly as Proposition [2, Proposition 6.4] is deduced from [2, Theorem 5.10].

Corollary 5.13. Let $G$ be a second countable compact quantum group, and $D$ be a strongly self-absorbing $C^{*}$-algebra. Then there exists at most one conjugacy class of $G$-actions on $D$ with the Rokhlin property.

The rest of this subsection is dedicated to the proof of Theorem 5.12.

We fix separable $G$-C $C^{*}$-algebras $(A, \alpha)$ and $(B, \beta)$, and homomorphisms $\phi, \phi_{1}, \phi_{2}:(A, \alpha) \rightarrow(B, \beta)$. We will use tacitly the fact that the unitary group of a unital $\mathrm{C}^{*}$-algebras is positively quantifier-free $\mathcal{L}^{\mathrm{C}^{*}, 1}$-definable with respect to the class of unital $\mathrm{C}^{*}$-algebras. Indeed, it is the zeroset of the stable positive quantifier-free $\mathcal{L}^{\mathrm{C}^{*}, 1}$-formula $\max \left\{\left\|x x^{*}-1\right\|,\left\|x^{*} x-1\right\|\right\}$.

Lemma 5.14. Suppose that $\beta \circ \phi \approx_{\mathrm{u}}(\mathrm{id} \otimes \phi) \circ \alpha$, and that $\beta$ has the Rokhlin property. Then for every finite subset $F$ of $A$ and every $\varepsilon>0$, there exists a unitary $v$ in the unitization of $C(G) \otimes B$ such that

$$
\sup _{x \in F}\|(\beta \circ \operatorname{Ad}(v) \circ \phi-(\operatorname{id} \otimes(\operatorname{Ad}(v) \circ \phi)) \circ \alpha)(x)\|<\varepsilon
$$

and

$$
\sup _{x \in F}(\|[\phi(x), v]\|-\|(\beta \circ \phi-(\mathrm{id} \otimes \phi) \circ \alpha)(x)\|)<\varepsilon .
$$

Proof. Fix a finite subset $F$ of $A$ and $\varepsilon>0$. By [2, Lemma 5.4], there exists a unitary $v$ in the unitization of $C(G) \otimes B$ such that

$$
\sup _{x \in F}\|((\Delta \otimes \beta) \circ \operatorname{Ad}(v) \circ(1 \otimes \phi)-(\operatorname{id} \otimes(\operatorname{Ad}(v) \circ(1 \otimes \phi))) \circ \alpha)(x)\|<\varepsilon
$$

and

$$
\sup _{x \in F}(\|[(1 \otimes \phi)(x), v]\|-\|(\beta \circ \phi-(\text { id } \circ \phi) \circ \alpha)(x) \|)<\varepsilon
$$


Since $\beta$ has the Rokhlin property, the map

$$
1 \otimes \operatorname{id}_{B}:(B, \beta) \rightarrow(C(G) \otimes B, \Delta \otimes \beta)
$$

is positively $\mathcal{L}_{G}^{\mathrm{C}^{*}}$-existential. Therefore its unique unital extension

$$
1 \otimes \operatorname{id}_{B}:(\tilde{B}, \beta) \rightarrow(C(G) \otimes \tilde{B}, \Delta \otimes \beta)
$$

is positively $\mathcal{L}_{G}^{\mathrm{C}^{*}, 1}$-existential. Since the conditions from Equation 2 and Equation 3 can be expressed by positive quantifier-free $\mathcal{L}_{G}^{\mathrm{C} * 1}$-formulas, we conclude that there exists a unitary $v \in \tilde{B}$ as wanted.

Lemma 5.15. Suppose that $\beta \circ \phi \approx_{\mathrm{u}}(\mathrm{id} \otimes \phi) \circ \alpha$, and $\beta$ has the Rokhlin property. Then there exists a $G$-equivariant *-homomorphism $\psi:(A, \alpha) \rightarrow(B, \beta)$ such that $\psi \approx_{\mathrm{u}} \phi$.

Proof. One replaces [2, Lemma 5.5] with Lemma 5.14 in the proof [2, Proposition 5.6].

We prove the following lemma directly using the definition of positively $\mathcal{L}_{G}^{\mathrm{C}^{*}}$-existential $G$-equivariant $*_{-}$ homomorphism.

Lemma 5.16. Suppose that $(C, \gamma)$ is a $G$-C $\mathrm{C}^{*}$-algebra, and $\psi:(B, \beta) \rightarrow(C, \gamma)$ is positively $\mathcal{L}_{G}^{\mathrm{C}^{*}}$-existential $G$-equivariant ${ }^{*}$-homomorphism. If $\psi \circ \phi_{1} \approx_{\mathrm{u}, G} \psi \circ \phi_{2}$ then $\phi_{1} \approx_{\mathrm{u}} \phi_{2}$.

Proof. Observe that the unital extension $\psi:(\tilde{B}, \beta) \rightarrow(\tilde{C}, \gamma)$ is a positively $\mathcal{L}_{G}^{\mathrm{C}^{*}, 1}$-existential $G$-equivariant *homomorphism. Fix $\varepsilon>0$ and a finite subset $F$ of $A$. Since by assumption $\psi \circ \phi_{1} \approx_{\mathrm{u}, G} \psi \circ \phi_{2}$, there exists $v \in \tilde{C}^{\gamma}$ such that

$$
\sup _{x \in F}\left\|v\left(\psi \circ \phi_{1}\right)(x) v^{*}-\left(\psi \circ \phi_{2}\right)(x)\right\|<\varepsilon .
$$

Since $\psi$ is a positively $\mathcal{L}_{G}^{\mathrm{C} *}$-existential $G$-equivariant ${ }^{*}$-homomorphism, there exists a unitary $u \in \tilde{B}^{\beta}$ such that $\sup _{x \in F}\left\|v \phi_{1}(x) v^{*}-\phi_{2}(x)\right\|<\varepsilon$. This concludes the proof.

The following proposition in the case when $G$ is coexact is [2, Corollary 5.9]. The proof is analogous, where one replaces [2, Proposition 5.8] with Lemma 5.16.

Proposition 5.17. Suppose that $G$ is a second countable compact quantum group, and $(A, \alpha)$ and $(B, \beta)$ are separable G-C*-algebras. Let $\phi_{1}, \phi_{2}:(A, \alpha) \rightarrow(B, \beta)$ be G-equivariant ${ }^{*}$-homomorphisms. If $\phi_{1} \approx_{\mathrm{u}} \phi_{2}$ and $(B, \beta)$ has the Rokhlin property, then $\phi_{1} \approx_{\mathrm{u}, G} \phi_{2}$.

Finally, using Proposition 5.17 instead of [2, Corollary 5.9], and Lemma 5.15 instead of [2, Corollary 5.6], one can prove Theorem 5.12 reasoning as in the proof of [2, Theorem 5.10].

As fruitful as the Rokhlin property is, it is also very rare. In fact, there are many very interesting $\mathrm{C}^{*}$ algebras that do not admit any Rokhlin action of a nontrivial compact quantum group. For example, we have the following result. For $\theta \in \mathbb{R} \backslash \mathbb{Q}$, we denote by $A_{\theta}$ the irrational rotation algebra. Also, we write $\mathcal{O}_{\infty}$ for the Cuntz algebra on infinitely many generators.

Proposition 5.18. Let $G$ be a nontrivial finite quantum group and let $\theta \in \mathbb{R} \backslash \mathbb{Q}$. There do not exist any actions of $G$ on either $A_{\theta}$ or $\mathcal{O}_{\infty}$ with the Rokhlin property.

Proof. For classical finite groups, this is well known; see, for example, Section 3 in [19]. Suppose that $G$ is not classical finite quantum group. Then $C(G)$ is a finite dimensional $\mathrm{C}^{*}$-algebra which is not commutative. Find $n \in \mathbb{N}$, with $n>1$, and orthogonal projections $p_{1}, \ldots, p_{n} \in C(G)$ which add up to $1_{C(G)}$ and are unitarily equivalent in $C(G)$.

Let $A$ be either $A_{\theta}$ or $\mathcal{O}_{\infty}$. Suppose that $\alpha$ is an action of $G$ on $A$, and assume by contradiction that $\alpha$ has the Rokhlin property. Therefore $\alpha:(A, \alpha) \rightarrow\left(C(G) \otimes A, \Delta \otimes \mathrm{id}_{A}\right)$ is positively $\mathcal{L}_{G}^{\mathrm{C}^{*}}$-existential. By considering the projections $p_{j} \otimes 1_{A} \in C(G) \otimes A$, we deduce that there exist orthogonal projections $q_{1}, \ldots, q_{n} \in A$ which add up to $1_{A}$ and are unitarily equivalent in $A$. In the case of $\mathcal{O}_{\infty}$, this would imply that the class of unit of $\mathcal{O}_{\infty}$ in its $K_{0}$-group is divisible by $n>1$, which is not true. For the case of $A_{\theta}$, and denoting its unique trace by $\tau$, we would get $1=\tau\left(1_{A_{\theta}}\right)=\sum_{j=1}^{n} \tau\left(q_{j}\right)=n \tau\left(q_{1}\right)$, since unitarily equivalent projections have the same value on traces. The range of $\tau$ on traces is known not to contain any rational which is not an integer, so this is again a contradiction. This finishes the proof.

Nonexistence results like the one just explained are the main motivation for introducing a more flexible notion, the Rokhlin dimension, which is the content of the next section. Despite not admitting any Rokhlin action, the algebras $\mathcal{O}_{\infty}$ and $A_{\theta}$ have many actions with finite Rokhlin dimension; see, for instance, [19]. 


\section{ORDER ZERO DIMENSION AND ROKHLIN DIMENSION}

6.1. Order zero dimension. The notion of positive $\mathcal{L}_{G}^{\mathrm{C}^{*}}$-existential $G$-equivariant ${ }^{*}$-homomorphism admits a natural generalization, which has been introduced in the classical setting in [22, Section 5]. We consider here its natural extension to compact quantum groups. In the following definition, given a ${ }^{*}$-homomorphism $\theta: A \rightarrow B$, we consider $B$ as an $A$-bimodule, with respect to the $A$-bimodule structure defined by $a \cdot b=\theta(a) b$ and $b \cdot a=b \theta(a)$ for every $a \in A$ and $b \in B$.

Definition 6.1. Let $G$ be either a discrete or a compact quantum group, let $(A, \alpha)$ and $(B, \beta)$ be $G$-C $C^{*}$-algebras. Fix a cardinal number $\kappa$ larger than the density characters of $A, B$, and $L^{2}(G)$, and a countably incomplete $\kappa$-good filter $\mathcal{F}$. We say that a *-homomorphism $\theta:(A, \alpha) \rightarrow(B, \beta)$ has $G$-equivariant order zero dimension at most $d$, written $\operatorname{dim}_{\mathrm{oz}}^{G}(\theta) \leq d$, if there exist $G$-equivariant completely positive contractive order zero $A$-bimodule maps $\psi_{0}, \ldots, \psi_{d}:(B, \beta) \rightarrow\left(\prod_{\mathcal{F}}^{G} A, \alpha_{\mathcal{F}}\right)$ such that the sum $\psi=\psi_{0}+\cdots+\psi_{d}$ is a contractive linear map such that the following diagram commutes

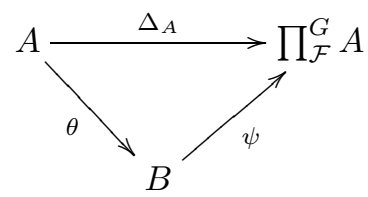

The $G$-equivariant order zero dimension $\operatorname{dim}_{\mathrm{oz}}^{G}(\theta)$ of $\theta$ is the least $d \in \mathbb{N}$ such that $\operatorname{dim}_{\mathrm{oz}}^{G}(\theta) \leq d$, if such a $d$ exists, and $\infty$ otherwise. The order zero dimension of a ${ }^{*}$-homomorphism between $\mathrm{C}^{*}$-algebras can be obtained as the particular instance of Definition 6.1 when $G$ is the trivial group.

Remark 6.2. Definition 6.1 does not depend on the choice of the countably incomplete $\kappa$-good ultrafilter $\mathcal{F}$. This can be seen, for instance, by considering the syntactic characterization presented in Remark 6.3 below. Furthermore, when $G$ is second countable, and $A, B$ are separable, one can choose $\mathcal{F}$ to be any countably incomplete filter, such as the filter of cofinite subsets of $\mathbb{N}$.

Remark 6.3. One can give a syntactic reformulation of the notion of $G$-equivariant order zero dimension. To this purpose, one can consider the ordered operator space language $\mathcal{L}^{\text {osos }}$ introduced in [22, Subsection 3.1 , and the order zero language $\mathcal{L}^{\mathrm{oz}}$ introduced in [22, Subsection 3.2]. Adding function symbols for the $A$-bimodule operations give the $A$-bimodule ordered operator space language $\mathcal{L}^{\text {osos, } A-A}$ and the $A$-bimodule order zero language $\mathcal{L}^{\mathrm{oz}, A-A}$. In both these languages, the distinguished symbol for the metric is replaced by pseudometric symbols $d_{F}$ for $F$ ranging among the finite subsets of $A$, to be interpreted as the pseudometric

$$
d_{F}(x, y)=\max \{\|a(x-y)\|,\|(x-y) a\|: a \in F\} .
$$

One can consider the $G$-equivariant version $\mathcal{L}_{G}^{\mathrm{osos}, A-A}$ and $\mathcal{L}_{G}^{\mathrm{oz}, A-A}$ for each of these languages, which are defined starting from these languages by adding symbols for a $G$-action as in Subsection 3.4. The $\mathcal{L}_{G}^{\text {osos, } A-A}$ morphisms between $G$-C $\mathrm{C}^{*}$-algebras are precisely the $G$-equivariant completely positive contractive $A$-bimodule maps, while the $\mathcal{L}_{G}^{\mathrm{oz}, A-A}$-morphisms are precisely the $G$-equivariant completely positive contractive order zero $A$-bimodule maps. One can then rephrase Definition 6.1 by asserting that the order zero dimension of a nondegenerate ${ }^{*}$-homomorphism $\theta:(A, \alpha) \rightarrow(B, \beta)$ is at most $d$ if and only if for every positive quantifier-free $\mathcal{L}_{G}^{\text {oz }, A-A}$-formula $\varphi(\bar{z}, \bar{y})$, for every positive quantifier-free $\mathcal{L}_{G}^{\text {osos, } A-A}$-formula $\psi(\bar{x}, \bar{z}, \bar{y})$, where the variables $\bar{z}$ have finite-dimensional $\mathrm{C}^{*}$-algebras as sorts, for every tuples $\bar{a}$ in $A, \bar{b}$ in $B$, and $\bar{w}$ in finite-dimensional $\mathrm{C}^{*}$-algebras, and for every $\varepsilon>0$, there exist tuples $\bar{c}_{0}, \ldots, \bar{c}_{d}$ in $A$ such that

$$
\psi\left(\bar{a}, \bar{w}, \bar{c}_{0}+\cdots+\bar{c}_{d}\right) \leq \psi(\theta(\bar{a}), \bar{w}, \bar{b})+\varepsilon \quad \text { and } \quad \varphi\left(\bar{w}, \bar{c}_{j}\right) \leq \varphi(\bar{w}, \bar{b})+\varepsilon \text { for } j=0,1, \ldots, d .
$$

If $A_{0}$ is a $G$-C $C^{*}$-subalgebra of $A$ containing an approximate unit for $A$, then one can replace $\mathcal{L}_{G}^{\text {osos, } A-A}$ and $\mathcal{L}_{G}^{\mathrm{oz}, A-A}$ with $\mathcal{L}_{G}^{\mathrm{osos}, A_{0}-A_{0}}$ and $\mathcal{L}_{G}^{\mathrm{oz}, A_{0}-A_{0}}$ in the discussion above.

Remark 6.4. Suppose that $\theta:(A, \alpha) \rightarrow(B, \beta)$ is a nondegenerate *-homomorphism. Fix a countably incomplete $\kappa$-good ultrafilter $\mathcal{U}$, where $\kappa$ is larger than the density character of $A, B$, and $L^{2}(G)$. Let also $A_{0}$ be a $G$-C ${ }^{*}$-subalgebra of $A$ containing an approximate unit for $A$. In the case when $G$ is compact, assume furthermore that $A_{0}$ is contained in the fixed point algebra (which is a nondegenerate $\mathrm{C}^{*}$-subalgebra of $A$ ). Following [30, Section 1] and [1, Remark 1.3], we consider the $G$-C $\mathrm{C}^{*}$-subalgebra $\overline{A_{0} \cdot \prod_{\mathcal{U}}^{G} A \cdot A_{0}}$ of $\prod_{\mathcal{U}}^{G} A$. It is easy to see that $\overline{A_{0} \cdot \prod_{\mathcal{U}}^{G} A \cdot A_{0}}$ can be identified with the ultrapower of $(A, \alpha)$ regarded as an $\mathcal{L}_{G}^{\mathrm{C} *}, A_{0}-A_{0}$-structure. It follows from the observations above that if $\operatorname{dim}_{\mathrm{oz}}^{G}(\theta) \leq d$, then there exist $G$-equivariant completely positive contractive 
order zero $A$-bimodule maps $\psi_{0}, \ldots, \psi_{d}: B \rightarrow \overline{A_{0} \cdot \prod_{\mathcal{U}}^{G} A \cdot A_{0}}$ such that $\psi:=\psi_{0}+\cdots+\psi_{d}$ is contractive and $\psi \circ \theta$ is the diagonal embedding from $A$ to $\overline{A_{0} \cdot \prod_{\mathcal{U}}^{G} A \cdot A_{0}}$.

Recall that a unital completely positive order zero map is a *-homomorphism. Next, we prove a similar result with 'unital' being replaced by 'nondegenerate'. If $\alpha$ is a $G$-action on $A$, then $\alpha$ admits a unique extension to a $G$-action on $\tilde{A}$, which we still denote by $\alpha$.

Lemma 6.5. Let $\phi: A \rightarrow B$ be a completely positive order zero map between $\mathrm{C}^{*}$-algebras. If $\phi$ is nondegenerate, then it is a ${ }^{*}$-homomorphism.

Proof. If $A$ is unital, then $\phi: A \rightarrow B \subset M(B)$ is unital, and the conclusion follows from the structure theorem for completely positive order zero maps from [50]. Suppose that $A$ is not unital, and let $\tilde{A}$ be its unitization. We let $B^{* *}$ be the second dual of $B$, which we identify with the enveloping von Neumann algebra of $B$. Fix an increasing approximate unit $\left(u_{j}\right)_{j \in J}$ for $A$, and set $g=\sup _{j \in J} \phi\left(u_{j}\right) \in B^{* *}$. By [50, Proposition 3.2], the (unique) linear map $\tilde{\phi}: \tilde{A} \rightarrow B^{* *}$ extending $\phi$ with $\phi(1)=g$, is completely positive of order zero. Since $\phi$ is nondegenerate, we must have $g=1 \in B^{* *}$. Therefore $\tilde{\phi}$ is a ${ }^{*}$-homomorphism, and hence so is $\phi$.

Lemma 6.6. Suppose that $G$ is a compact or discrete quantum group, and $(A, \alpha)$ is a $G$-C $C^{*}$-algebra. The inclusion map $(A, \alpha) \hookrightarrow(\tilde{A}, \alpha)$ is positively $\mathcal{L}_{G}^{\mathrm{C}^{*}, A-A}$-existential.

Proof. Consider an approximate unit $\left(u_{j}\right)_{j \in J}$ for $A$. Fix a quantifier-free $\mathcal{L}_{G}^{\mathrm{C} * A-A}$-formula, $\varphi(\bar{x}, \bar{y})$, and a tuple $\bar{a}$ in $A$. If $\left(b_{k}+\lambda_{k} 1\right)_{k=1}^{n}$ is a tuple in $\tilde{A}$ satisfying the condition $\varphi(\bar{a}, \bar{y})<r$, then, for a suitable $j \in J$, $\left(b_{k}+\lambda_{k} u_{j}\right)_{k=1}^{n}$ is a tuple in $A$ satisfying the same condition.

Proposition 6.7. Let $G$ be either a compact or discrete quantum group, and let $\theta:(A, \alpha) \rightarrow(B, \beta)$ be an injective *-homomorphism between $G$ - $C^{*}$-algebras.

(1) If $(C, \gamma)$ is a $G$ - $C^{*}$-algebra, and $\psi:(B, \beta) \rightarrow(C, \gamma)$ is a *-homomorphism, then

$$
\operatorname{dim}_{\mathrm{oz}}^{G}(\psi \circ \theta)+1 \leq\left(\operatorname{dim}_{\mathrm{oz}}^{G}(\psi)+1\right)\left(\operatorname{dim}_{\mathrm{oz}}^{G}(\theta)+1\right) .
$$

(2) If $D$ is any $C^{*}$-algebra, then $\operatorname{dim}_{\mathrm{oz}}^{G}\left(\theta \otimes \operatorname{id}_{D}\right) \leq \operatorname{dim}_{\mathrm{oz}}^{G}(\theta)$.

(3) Let $I$ be a directed set, and let $\left(\left(A_{i}, \alpha_{i}\right), \theta_{i j}\right)_{i, j \in I}$ be a direct system of $G$ - $C^{*}$-algebras. For every $i \in I$, let $\theta_{i \infty}:\left(A_{i}, \alpha\right) \rightarrow\left(\stackrel{\lim }{\longrightarrow} A_{i}, \lim _{\longrightarrow} \alpha_{i}\right)$ be the canonical ${ }^{*}$-homomorphism. Then $\operatorname{dim}_{\mathrm{oz}}^{G}\left(\theta_{i \infty}\right) \leq$ $\lim \sup _{j} \operatorname{dim}_{\mathrm{oz}}^{G}\left(\theta_{i j}\right)$.

(4) Let I be a directed set, and for $k=0,1$, let $\left(\left(A_{i}^{(k)}, \alpha_{i}^{(k)}\right), \theta_{i j}^{(k)}\right)_{i, j \in I}$ be a direct system of $G$ - $C^{*}$-algebras. Let $\left(\eta_{i}:\left(A_{i}^{(0)}, \alpha_{i}^{(0)}\right) \rightarrow\left(A_{i}^{(1)}, \alpha_{i}^{(1)}\right)\right)$ be a compatible family of *-homomorphism. Then $\operatorname{dim}_{\mathrm{oz}}^{G}\left(\underline{\lim } \eta_{i}\right) \leq$ $\lim \sup _{i \in I} \operatorname{dim}_{\mathrm{oz}}^{G}\left(\eta_{i}\right)$.

(5) Suppose that $S$ is a positively quantifier-free $\mathcal{L}_{G}^{\mathrm{osos}, A-A}$-definable $G$ - $C^{*}$-subalgebra relative the class $\mathcal{C}$ of $G$ - $C^{*}$-algebras that contain $(A, \alpha)$. Then $\theta$ maps $S^{(A, \alpha)}$ to $S^{(B, \beta)}$, and $\operatorname{dim}_{\mathrm{oz}}^{G}\left(\left.\theta\right|_{S^{(A, \alpha)}}\right) \leq \operatorname{dim}_{\mathrm{oz}}^{G}(\theta)$.

Proof. (1)-(4) can be proved similarly as for classical groups; see [22, Proposition 5.4]. (5) is an easy consequence of the definition.

The following is one of our main results regarding order zero dimension. In the proof of part (5), we will use the fact that if $\psi: A \rightarrow B$ is a completely positive contractive order zero map, then $\psi(a) \psi(b c)=\psi(a b) \psi(c)$ for all $a, b, c \in A$. This fact follows easily by considering the induced *-homomorphism $C_{0}((0,1]) \otimes A \rightarrow B[50$, Corollary 4.1].

Theorem 6.8. Let $G$ be either a compact or discrete quantum group, and let $\theta:(A, \alpha) \rightarrow(B, \beta)$ be a nondegenerate injective *-homomorphism. Then:

(1) $\operatorname{dim}_{\mathrm{oz}}^{G}(\theta)=0$ if and only if $\theta$ is positively $\mathcal{L}_{G}^{C^{*}}$-existential.

(2) The G-equivariant order zero dimension of $\tilde{\theta}:(\tilde{A}, \alpha) \rightarrow(\tilde{B}, \beta)$ is equal to $\operatorname{dim}_{\mathrm{oz}}^{G}(\theta)$.

(3) The $\check{G}$-equivariant order zero dimension of $\left.G \ltimes \theta:\left(G \ltimes_{\alpha, \mathrm{r}} A, \check{\alpha}\right) \rightarrow\left(G \ltimes_{\beta, \mathrm{r}} B, \check{\beta}\right)\right)$ is less than or equal to $\operatorname{dim}_{\mathrm{oz}}^{G}(\theta)$.

(4) If $(B, \beta)$ is free, and $\operatorname{dim}_{G}^{\mathrm{oz}}(\theta)<+\infty$, then $(A, \alpha)$ is free.

Proof. We prove the theorem for compact $G$, since the case of discrete $G$ is analogous.

(1): It is obvious that if $\theta$ is positively $\mathcal{L}_{G}^{\mathrm{C}^{*}}$-existential, then $\operatorname{dim}_{\mathrm{oz}}^{G}(\theta)=0$. Conversely, suppose that $\operatorname{dim}_{\mathrm{oz}}^{G}(\theta)=0$. Fix a countably incomplete $\kappa$-good ultrafilter $\mathcal{U}$, where $\kappa$ is larger than the density character of $A$, 
$B$, and $L^{2}(G)$. Then by Remark 6.4 , there exists a completely positive contractive order zero $A$-bimodule map $\psi: B \rightarrow \overline{A \cdot \prod_{\mathcal{U}}^{G} A \cdot A}$ such that $\psi \circ \phi: A \rightarrow \overline{A \cdot \prod_{\mathcal{U}}^{G} A \cdot A}$ is the diagonal inclusion. Since $\phi$ is nondegenerate and $\psi$ is an $A$-bimodule map, $\psi$ is nondegenerate. Therefore $\psi$ is a ${ }^{*}$-homomorphism, witnessing that $\theta$ is positively $\mathcal{L}_{G}^{\mathrm{C}^{*}}$-existential.

(2): We can assume that $\theta: A \rightarrow B$ is the inclusion map. Suppose that $\operatorname{dim}_{\mathrm{oz}}^{G}(\theta) \leq d$. By Lemma 6.6 and Remark 6.4, there exist $G$-equivariant completely positive order zero $A$-bimodule maps

$$
\psi_{0}, \ldots, \psi_{d}: \tilde{B} \rightarrow \overline{A^{\alpha} \cdot \prod_{\mathcal{U}}^{G} A \cdot A^{\alpha}} \subseteq \overline{A^{\alpha} \cdot \prod_{\mathcal{U}}^{G} \tilde{A} \cdot A^{\alpha}}
$$

such that $\psi=\psi_{0}+\cdots+\psi_{d}$ is contractive and $\left.\psi \circ \tilde{\theta}\right|_{A}: A \rightarrow \overline{A^{\alpha} \cdot \prod_{\mathcal{U}}^{G} A \cdot A^{\alpha}}$ is the diagonal embedding. Since $\psi_{0}, \ldots, \psi_{d}$ are $A$-bimodule maps, given $a \in A^{\alpha}$ we have

$$
a \psi(1)=\psi(a 1)=\psi(a)=a,
$$

and similarly $\psi(1) a=a$. This shows that $\psi(1)=1$ and that

$$
\psi \circ \tilde{\theta}: \tilde{A} \rightarrow \overline{A^{\alpha} \cdot \prod_{\mathcal{U}}^{G} \tilde{A} \cdot A^{\alpha}} \subseteq \prod_{\mathcal{U}}^{G} \tilde{A}
$$

is the diagonal embedding. We also have, for $j=0,1, \ldots, d$.

$$
(a+\lambda 1) \psi_{j}(b+\mu 1)=a \psi_{j}(b+\mu 1)+\lambda \psi_{j}(b+\mu 1)=\psi_{j}(a b+\mu a+\lambda b+\lambda \mu 1)=\psi_{j}((a+\lambda 1)(b+\mu 1)) .
$$

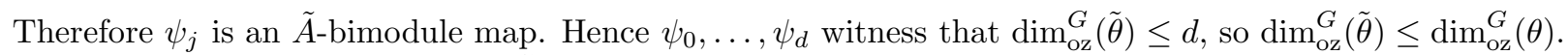

Conversely, suppose that $\operatorname{dim}_{\mathrm{oz}}^{G}(\tilde{\theta}) \leq d$. Observe that $A^{\alpha}$ is a closed two-sided ideal of the fixed point algebra of $(\tilde{A}, \alpha)$. Therefore, by Remark 6.4 , there exist $G$-equivariant completely positive contractive order zero $A$ bimodule maps $\psi_{0}, \ldots, \psi_{d}: \tilde{B} \rightarrow \overline{A^{\alpha} \cdot \prod_{\mathcal{U}}^{G} \tilde{A} \cdot A^{\alpha}}$. Note that $\overline{A^{\alpha} \cdot \prod_{\mathcal{U}}^{G} \tilde{A} \cdot A^{\alpha}}=\overline{A^{\alpha} \cdot \prod_{\mathcal{U}}^{G} A \cdot A^{\alpha}}$. Therefore the restriction of the maps $\psi_{0}, \ldots, \psi_{d}$ to $B$ witness that $\operatorname{dim}_{\mathrm{oz}}^{G}(\theta) \leq d, \operatorname{so} \operatorname{dim}_{\mathrm{oz}}^{G}(\theta) \leq \operatorname{dim}_{\mathrm{oz}}^{G}(\tilde{\theta})$, as desired.

(3): Suppose that $\operatorname{dim}_{\mathrm{oz}}^{G}(\theta)=d$. Let us first consider the case when $A, B$ are unital $\mathrm{C}^{*}$-algebras, in which case $\theta$ is a unital *-homomorphism. Fix a cardinal $\kappa$ larger than the density characters of $A, B$, and $L^{2}(G)$, and a countably incomplete $\kappa$-good ultrafilter $\mathcal{U}$. Let $\psi_{0}, \ldots, \psi_{d}: B \rightarrow \prod_{\mathcal{U}}^{G} A$ be maps as in the definition of $\operatorname{dim}_{\mathrm{oz}}^{G}(\theta) \leq d$. Consider the following maps:

- the $\check{G}$-equivariant completely positive contractive $A$-bimodule maps $G \ltimes \psi_{j}: G \ltimes_{\beta} B \rightarrow G \ltimes_{\alpha}\left(\prod_{\mathcal{U}}^{G} A\right)$ for $j=0, \ldots, d$ obtained as in Lemma 4.3 ;

- the $\breve{G}$-equivariant *-homomorphism $G \ltimes \theta: G \ltimes_{\alpha} A \rightarrow G \ltimes_{\beta} B$; and

- the $\check{G}$-equivariant injective ${ }^{*}$-homomorphism $\Psi: G \ltimes_{\alpha}\left(\prod_{\mathcal{U}}^{G} A\right) \rightarrow \prod_{\mathcal{U}}^{\check{G}} A$ from Proposition 4.4.

Then $G \ltimes \psi=\sum_{j=0}^{d} G \ltimes \psi_{j}$ is a $\check{G}$-equivariant completely positive $A$-bimodule map satisfying

$$
\Psi \circ(G \ltimes \psi) \circ(G \ltimes \theta)=\Delta_{A}: A \rightarrow \prod_{\mathcal{U}}^{\check{G}} A .
$$

Since $G \ltimes \psi$ is completely positive, we have $\|G \ltimes \psi\|=\|(G \ltimes \psi)(1)\|=1$. Therefore $\operatorname{dim}_{\mathrm{oz}}^{\check{G}}(G \ltimes \theta) \leq d$.

Consider now the case when $A$ and $B$ are not necessarily unital. Then $\operatorname{dim}_{\mathrm{oz}}^{G}(\tilde{\theta}) \leq d$ by (2). By applying the result to the unital case, we have $\operatorname{dim}_{\mathrm{oz}}^{\hat{G}}(G \ltimes \tilde{\theta}) \leq d$. Observe now that $G \ltimes_{\alpha} A \subseteq G \ltimes_{\alpha} \tilde{A}$ and $G \ltimes_{\beta} B \subseteq G \ltimes \tilde{B}$ are positively quantifier-free $\mathcal{L}_{\tilde{G}}^{A^{\alpha}-A^{\alpha}}$-definable with respect to the class of $\check{G}$-C $\mathrm{C}^{*}$-algebras of the form $G \ltimes \gamma C$ for some $G$-C $C^{*}$-algebra $(C, \gamma)$ such that $(A, \alpha)$ embeds equivariantly into $(C, \gamma)$. Therefore, by part (5) of Proposition 6.7, the restriction $G \ltimes \theta: G \ltimes_{\alpha} A \rightarrow G \ltimes_{\beta} B$ also has $\check{G}$-equivariant order zero dimension at most $d$ in view of the semantic characterization of $\breve{G}$-equivariant order zero dimension from Remark 6.3. We conclude that $\operatorname{dim}_{\mathrm{oz}}^{\breve{G}}(G \ltimes \theta) \leq \operatorname{dim}_{\mathrm{oz}}^{G}(\theta)$.

(4): We can assume that $A \subseteq B$ is a nondegenerate $G$-C*-subalgebra, and $\theta: A \rightarrow B$ is the inclusion map. Let $x \in C(G) \otimes A$, and $\varepsilon>0$. Using freeness for $\beta$, find $n \in \mathbb{N}$, and tuples $b_{1}, \ldots, b_{n}, c_{1}, \ldots, c_{n} \in B$ with

$$
\left\|x-\sum_{k=1}^{n} \beta\left(b_{k}\right)\left(1 \otimes c_{k}\right)\right\|<\varepsilon / 2 .
$$

Set $M=\max _{k=1, \ldots, n}\left\|b_{k}\right\|$ and $d=\operatorname{dim}_{\mathrm{oz}}^{G}(\theta)<\infty$. Since $A \subseteq B$ is nondegenerate, choose $u \in A_{+}$satisfying

$$
\|x(1 \otimes u)-x\|<\varepsilon / 3, \text { and }\left\|c_{k} u-c_{k}\right\|<\varepsilon /(3(d+1) n M)
$$

for all $k=1, \ldots, n$. 
Let $\kappa$ be a cardinal larger than the density characters of $A, B$, and $L^{2}(G)$. Find $G$-equivariant completely positive order zero maps $\psi_{0}, \ldots, \psi_{d}: B \rightarrow \prod_{\mathcal{U}}^{G} A$ as in the definition of order zero dimension. For $j=0, \ldots, d$, let $\pi_{j}: B \rightarrow \prod_{\mathcal{U}}^{G} A$ be the completely positive contractive order zero map given by $\pi_{j}=\psi_{j}^{1 / 2}$, using functional calculus for order zero maps (see [50, Corollary 3.2]). It is easily checked that $\pi_{j}$ is again a $G$-equivariant $A$-bimodule map.

For $j=0, \ldots, d$ and $k=1, \ldots, n$, set $b_{j, k}=\pi_{j}\left(b_{k}\right)$ and $c_{j, k}=\psi_{j}\left(c_{k}\right)$. For $j=0, \ldots, d$, denote by $\phi_{j}: C(G) \otimes$ $B \rightarrow C(G) \otimes \prod_{\mathcal{U}}^{G} A$ the $G$-equivariant completely positive order zero $A$-bimodule map $\phi_{j}=\operatorname{id}_{C(G)} \otimes \pi_{j}$. In the rest of this proof, for elements $e$ and $f$ in some $\mathrm{C}^{*}$-algebra and $\delta>0$, we write $e \approx_{\delta} f$ to mean $\|e-f\|<\delta$. By the comments before this proposition, we have

$$
\begin{aligned}
\phi_{j}\left(\beta\left(b_{k}\right)\left(1 \otimes c_{k}\right)\right) \phi_{j}(1 \otimes u) & =\phi_{j}\left(\beta\left(b_{k}\right)\right) \phi_{j}\left(1 \otimes c_{k} u\right) \\
& =\alpha_{\mathcal{U}}\left(b_{j, k}\right)\left(1 \otimes c_{j, k} u\right) \\
& \approx_{\varepsilon /(3(d+1) n)} \alpha_{\mathcal{U}}\left(b_{j, k}\right)\left(1 \otimes c_{j, k}\right) .
\end{aligned}
$$

In the following computation, we use the observation above at the first step; the definition of the elements $b_{k}$ and $c_{k}$ at the third; the fact that $u$ has a square root at the fourth, together with the comments before this proposition regarding order zero maps; the fact that $\phi_{j}$ is a $(C(G) \otimes A)$-bimodule map at the fifth; the definition of $\phi_{j}$ at the seventh; and the properties of the maps $\psi_{j}$ at the eighth:

$$
\begin{aligned}
\sum_{j=0}^{d} \sum_{k=1}^{n} \alpha_{\mathcal{U}}\left(b_{j, k}\right)\left(1 \otimes c_{j, k}\right) & \approx_{\varepsilon / 3} \sum_{j=0}^{d} \sum_{k=1}^{n} \phi_{j}\left(\beta\left(b_{k}\right)\left(1 \otimes c_{k}\right)\right) \phi_{j}(1 \otimes u)=\sum_{j=0}^{d} \phi_{j}\left(\sum_{k=1}^{n} \beta\left(b_{k}\right)\left(1 \otimes c_{k}\right)\right) \phi_{j}(1 \otimes u) \\
& \approx_{\varepsilon / 3} \sum_{j=0}^{d} \phi_{j}(x) \phi_{j}(1 \otimes u)=\sum_{j=0}^{d} \phi_{j}\left(x\left(1 \otimes u^{1 / 2}\right)\right) \phi_{j}\left(1 \otimes u^{1 / 2}\right) \\
& =x \sum_{j=0}^{d} \phi_{j}\left(1 \otimes u^{1 / 2}\right) \phi_{j}\left(1 \otimes u^{1 / 2}\right)=x \sum_{j=0}^{d} \phi_{j}^{2}(1 \otimes u) \\
& =x \sum_{j=0}^{d}\left(\operatorname{id}_{C(G)} \otimes \psi\right)(1 \otimes u)=x(1 \otimes u) \approx_{\varepsilon / 3} x
\end{aligned}
$$

We conclude that $\left\|x-\sum_{j=0}^{d} \sum_{k=1}^{n} \alpha_{\mathcal{U}}\left(b_{j, k}\right)\left(1 \otimes c_{j, k}\right)\right\|<\varepsilon$. Since the diagonal embedding of $A \rightarrow \prod_{\mathcal{U}}^{G} A$ is positively $\mathcal{L}_{G}^{\mathrm{C}^{*}}$-existential, we conclude that there exist $\tilde{b}_{j, k}, \tilde{c}_{j, k} \in A$ satisfying

$$
\left\|x-\sum_{k=1}^{n} \sum_{j=0}^{d} \alpha\left(\tilde{b}_{j, k}\right)\left(1 \otimes \tilde{c}_{j, k}\right)\right\|<\varepsilon .
$$

This shows that $(A, \alpha)$ is free.

Theorem 6.9. Let $G$ be a compact quantum group, and let $\theta:(A, \alpha) \rightarrow(B, \beta)$ be a nondegenerate injective *-homomorphism. Then:

(1) The G-equivariant order zero dimension of $\operatorname{id}_{\mathbb{K}_{G}} \otimes \theta:\left(\mathbb{K}_{G} \otimes A, \alpha_{\mathbb{K}}\right) \rightarrow\left(\mathbb{K}_{G} \otimes B, \beta_{\mathbb{K}}\right)$ is equal to $\operatorname{dim}_{\mathrm{oz}}^{G}(\theta)$.

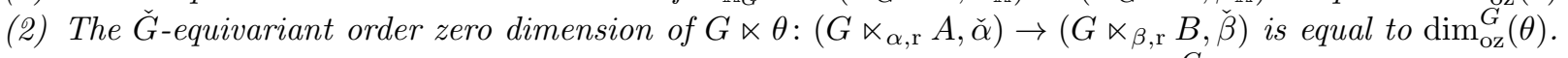

(3) The G-equivariant order zero dimension of $\left.\theta\right|_{A^{\alpha}}$ is less than or equal to $\operatorname{dim}_{\mathrm{oz}}^{G}(\theta)$.

Proof. (1): The proof of this fact is identical to the proof of part (2) of Proposition 5.5.

(2): One inequality follows from Theorem 6.8. As in the proof of part (3) of Proposition 5.5, one can now deduce that in fact equality holds using Item (1) above and the Baaj-Skandalis-Takesaki-Takai duality for compact quantum groups.

(3): This is a particular instance of part (5) of Proposition 6.7 in the case of the fixed point subalgebra, which is an $\mathcal{L}_{G}^{\text {osos }}$-definable $G$-C $\mathrm{C}^{*}$-subalgebra relative to the class of $G$-C ${ }^{*}$-algebras when $G$ is a compact quantum group.

The following preservation result for *-homomorphisms with finite order zero dimension has been established in $\left[22\right.$, Proposition 5.22]. Given a $\mathrm{C}^{*}$-algebra $A$, we let $\operatorname{dim}_{\text {nuc }}(A)$ be the nuclear dimension of $A[51]$, and $\operatorname{dr}(A)$ be the decomposition rank of $A$ [31, Definition 3.1]. 
Proposition 6.10. Let $A, B$ be $C^{*}$-algebras, and $\theta: A \rightarrow B$ be a ${ }^{*}$-homomorphism. Then

$$
\operatorname{dim}_{\text {nuc }}(A)+1 \leq\left(\operatorname{dim}_{\mathrm{oz}}(\theta)+1\right)\left(\operatorname{dim}_{\mathrm{nuc}}(B)+1\right)
$$

and

$$
\operatorname{dr}(A)+1 \leq\left(\operatorname{dim}_{\mathrm{oz}}(\theta)+1\right)(\operatorname{dr}(B)+1) .
$$

More generally, Proposition 6.10 applies to any dimension function for (nuclear) $\mathrm{C}^{*}$-algebras that is (nuclearly) positively $\forall \exists$-axiomatizable in the sense of [22, Definition 5.15, Definition 5.16.].

6.2. Rokhlin dimension. In this subsection we fix a compact quantum group $G$. We consider $C(G) \otimes A$ as a $G$-C ${ }^{*}$-algebra with respect to the action given by $\Delta \otimes \mathrm{id}_{A}$.

Definition 6.11. The Rokhlin dimension $\operatorname{dim}_{\mathrm{Rok}}(A, \alpha)$ of a $G$-C $\mathrm{C}^{*}$-algebra $(A, \alpha)$, is the $G$-equivariant order zero dimension of $\alpha:(A, \alpha) \rightarrow\left(C(G) \otimes A, \Delta \otimes \mathrm{id}_{A}\right)$.

It follows from [2, Lemma 1.24] and [22, Lemma 5.13] that when $G$ is classical, Definition 6.11 recovers the usual notion of Rokhlin dimension for $G$-C $C^{*}$-algebras from [21, Definition 3.2].

The following is the main technical fact for actions with finite Rokhlin dimension.

Theorem 6.12. Let $(A, \alpha)$ be a $G$ - $C^{*}$-algebra. Then

$$
\operatorname{dim}_{\mathrm{oz}}\left(A^{\alpha} \hookrightarrow A\right) \leq \operatorname{dim}_{\text {Rok }}(A, \alpha)=\operatorname{dim}_{\mathrm{oz}}^{\check{G}}\left(G \ltimes_{\alpha} A \hookrightarrow \mathbb{K}_{\check{G}} \otimes A\right) .
$$

Here $\mathbb{K}_{\check{G}} \otimes A$ is regarded as a $\check{G}$-C $C^{*}$-algebra endowed with the stabilization of the trivial action of $\check{G}$ on $A$.

Proof. Let $d$ be the Rokhlin dimension of $(A, \alpha)$. We consider $\alpha$ as a $G$-equivariant *-homomorphism $\alpha$ : $(A, \alpha) \rightarrow\left(C(G) \otimes A, \Delta \otimes \mathrm{id}_{A}\right)$. We thus have that $d$ is equal to the $G$-equivariant order zero dimension of $\alpha$. By part $(3)$ of Theorem $6.9, \operatorname{dim}_{\mathrm{oz}}^{G}\left(\left.\alpha\right|_{A^{\alpha}}\right) \leq \operatorname{dim}_{\mathrm{oz}}^{G}(\alpha)=\operatorname{dim}_{\mathrm{Rok}}(A, \alpha)$. The restriction $\left.\alpha\right|_{A^{\alpha}}$ is the embedding $A^{\alpha} \hookrightarrow 1 \otimes A \subseteq C(G) \otimes A$, so this proves the first equality.

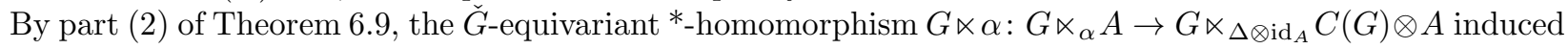
by $\alpha$ is equal to $d$. A particular instance of the Baaj-Skandalis-Takesaki-Takai duality for compact quantum groups yields a $\check{G}$-equivariant ${ }^{*}$-isomorphism $\rho: G \ltimes_{\Delta \otimes \operatorname{id}_{A}}(C(G) \otimes A) \rightarrow \mathbb{K}_{G} \otimes A$ such that $\rho \circ(G \ltimes \alpha): G \ltimes{ }_{\alpha} A \rightarrow$ $\mathbb{K}_{G} \otimes A$ is the canonical inclusion. This shows the $\check{G}$-equivariant ${ }^{*}$-homomorphism $G \ltimes_{\alpha} A \hookrightarrow \mathbb{K}_{G} \otimes A$ also has $\check{G}$-equivariant order zero dimension $d$.

Corollary 6.13. Suppose that $G$ is a compact quantum group, and $(A, \alpha)$ is a $G$ - $C^{*}$-algebra. Then

$$
\operatorname{dim}_{\text {nuc }}\left(A^{\alpha}\right)+1 \leq\left(\operatorname{dim}_{\text {Rok }}(A, \alpha)+1\right)\left(\operatorname{dim}_{\text {nuc }}(A)+1\right)
$$

and

$$
\operatorname{dr}\left(G \ltimes_{\alpha} A\right)+1 \leq\left(\operatorname{dim}_{\text {Rok }}(A, \alpha)+1\right)(\operatorname{dr}(A)+1) .
$$

Proof. This is an immediate consequence of Theorem 6.12 and Proposition 6.10.

Remark 6.14. Let $\theta:(A, \alpha) \rightarrow(B, \beta)$ be a $G$-equivariant *-homomorphism. Using part (1) of Proposition 6.7 at the fourth step, we get

$$
\begin{aligned}
\operatorname{dim}_{\text {Rok }}(A, \alpha)+1 & =\operatorname{dim}_{\mathrm{oz}}^{G}(\alpha)+1 \leq \operatorname{dim}_{\mathrm{oz}}^{G}((\mathrm{id} \otimes \theta) \circ \alpha)+1=\operatorname{dim}_{\mathrm{oz}}^{G}(\beta \circ \theta)+1 \\
& \leq\left(\operatorname{dim}_{\mathrm{oz}}^{G}(\beta)+1\right)\left(\operatorname{dim}_{\mathrm{oz}}^{G}(\theta)+1\right)=\left(\operatorname{dim}_{\mathrm{Rok}}(B, \beta)+1\right)\left(\operatorname{dim}_{\mathrm{oz}}^{G}(\theta)+1\right) .
\end{aligned}
$$

The following dimensional inequalities follow from the remark above and [22, Theorem 5.40 and Theorem 4.41]. We denote by $\mathcal{Z}$ the Jiang-Su algebra, and by $\mathcal{O}_{2}$ and $\mathcal{O}_{\infty}$ the Cuntz algebras on two and infinitely many generators, respectively.

Theorem 6.15. Let $(A, \alpha)$ be a $G$-C $C^{*}$-algebra, and let $U$ be UHF-algebra of infinite type. Then

$$
\operatorname{dim}_{\text {Rok }}\left(A \otimes \mathcal{Z}, \alpha \otimes \operatorname{id}_{\mathcal{Z}}\right) \leq 2 \operatorname{dim}_{\text {Rok }}\left(A \otimes U, \alpha \otimes \operatorname{id}_{U}\right)+1
$$

and

$$
\operatorname{dim}_{\text {Rok }}\left(A \otimes \mathcal{O}_{\infty}, \alpha \otimes \operatorname{id}_{\mathcal{O}_{\infty}}\right) \leq 2 \operatorname{dim}_{\text {Rok }}\left(A \otimes \mathcal{O}_{2}, \alpha \otimes \operatorname{id}_{\mathcal{O}_{2}}\right)+1
$$

Finally, we show that actions with finite Rokhlin dimension are free. This result is new even for actions of classical finite groups. Previous partial results were considerably more technical, and the quantum group perspective makes the argument significantly more transparent.

Theorem 6.16. Let $(A, \alpha)$ be a $G$ - $C^{*}$-algebra. If $\operatorname{dim}_{\text {Rok }}(\alpha)<\infty$, then $\alpha$ is free.

Proof. By Example 3.9, the $G$-C $\mathrm{C}^{*}$-algebra $C(G) \otimes A$ is free. Since $\alpha: A \rightarrow C(G) \otimes A$ has finite $G$-equivariant order zero dimension, the result follows from part (4) of Theorem 6.8. 
6.3. Duality. One can isolate a notion of dimension for actions of discrete quantum groups, which is dual to the notion of Rokhlin dimension for actions of compact quantum groups. Suppose that $G$ is a compact quantum group, and let $(A, \alpha)$ be a $\check{G}-C^{*}$-algebra. Consider the reduced crossed product $\check{G} \ltimes_{\alpha, \mathrm{r}} A$, and the canonical inclusion $\iota_{C(G)}: C(G) \rightarrow M\left(\check{G} \ltimes_{\alpha, \mathrm{r}} A\right)$. Denote by $V$ the fundamental unitary of $G$. Consider now the unitary

$$
V_{\alpha}:=\left(\operatorname{id}_{c_{0}(\check{G})} \otimes \iota_{C(G)}\right)(V) \in M\left(c_{0}(\check{G}) \otimes\left(\check{G} \ltimes_{\alpha, \mathrm{r}} A\right)\right) .
$$

Then the map $\operatorname{Ad}\left(V_{\alpha}^{*}\right)$ turns $M\left(\check{G} \ltimes_{\alpha, \mathrm{r}} A\right)$ into a $\check{G}$-C $\mathrm{C}^{*}$-algebra.

Definition 6.17. Let $(A, \alpha)$ be a $\check{G}$-C ${ }^{*}$-algebra. The representation dimension $\operatorname{dim}_{\text {rep }}(A, \alpha)$ of $(A, \alpha)$ is the $\check{G}$-equivariant order zero dimension of the canonical embedding $(A, \alpha) \rightarrow\left(\check{G} \ltimes_{\alpha, \mathrm{r}} A, \operatorname{Ad}\left(V_{\alpha}^{*}\right)\right)$.

In the case of dimension zero, and when $G$ is coexact and $A$ is separable, Definition 6.17 recovers the notion of spatial approximate representability from [2, Definition 4.7]. The following result generalizes [2, Theorem $4.12]$, which is then the case of dimension zero.

Theorem 6.18. Let $G$ be a compact quantum group and let $(A, \alpha)$ be a $G$ - $C^{*}$-algebra. Then

$$
\operatorname{dim}_{\text {Rok }}(A, \alpha)=\operatorname{dim}_{\text {rep }}\left(G \rtimes_{\alpha, r} A, \check{\alpha}\right) .
$$

Proof. Using the identifications from [2, Proposition 4.10, Proposition 4.11], the conclusion follows from part (2) of Theorem 6.9 applied to $\alpha: A \rightarrow C(G) \otimes A$.

\section{Appendix}

We recall here the fundamental notions concerning first order logic for metric structure. A good introduction to this subject can be found in [3]. The systematic study of $\mathrm{C}^{*}$-algebras from the perspective of model theory has been undertaken in [12]. We will consider the framework of languages with domains of quantification as introduced in [15], which is particularly suitable for dealing with structures from functional analysis. In fact, we will consider a slightly more general setting, which is necessary for our purposes, where the interpretation of the domains of quantifications of a given sort are only required to be dense in the interpretation of the sort. We will also consider the situation where, rather than a single distinguished metric single, the language contains an upward directed collection of pseudometric symbols. This is useful when considering structures such as modules and bimodules. It is clear that all the results of [15] go through in this slightly more general setting.

A.1. Syntax. A language $\mathcal{L}$ is given by:

- a collection of sorts $\mathcal{S}$,

- a collection of function symbols $f$,

- a collection of relation symbols $R$,

- for each sort $\mathcal{S}$, an ordered, upward directed collection of domains of quantification $D$ for $\mathcal{S}$,

- for each sort $\mathcal{S}$, a collection of variables of sort $\mathcal{S}$,

- for each sort $\mathcal{S}$, an ordered, upward directed collection of pseudometric symbols $d_{\mathcal{S}}$ of arity 2 with input sorts all equal to $\mathcal{S}$,

- for each function and relation symbol $B$, a natural number $n_{B}$ (its arity),

- for each function symbol $f$, a distinguished tuple $\mathcal{S}_{1}^{f}, \ldots, \mathcal{S}_{n_{f}}^{f}$ of input sorts and an output sort $\mathcal{S}^{f}$,

- for each relation symbol $R$, a distinguished tuple $\mathcal{S}_{1}^{R}, \ldots, \mathcal{S}_{n_{R}}^{R}$ of input sorts,

- for each function symbol $f$ and for each tuple $D_{1}, \ldots, D_{n_{f}}$ of domains of quantifications for $\mathcal{S}_{1}^{f}, \ldots, \mathcal{S}_{n_{f}}^{f}$, and for each tuple of pseudometric symbols $d_{1}, \ldots, d_{n_{f}}$ of sort $\mathcal{S}_{1}, \ldots, \mathcal{S}_{n_{f}}$, distinguished domains of quantification $D_{D_{1}, \ldots, D_{n_{f}}}^{f}$ and a distinguished pseudometric symbol $d_{D_{1}, \ldots, D_{n_{f}}, d_{1}, \ldots, d_{n_{f}}}^{f}$ for the output sort $\mathcal{S}^{f}$ of $f$, and a distinguished continuity modulus $\varpi_{D_{1}, \ldots, D_{n_{f}}, d_{1}, \ldots, d_{n_{f}}}^{f}$;

- for each relation symbol $R$ and for each tuple $D_{1}, \ldots, D_{n_{R}}$ of domains of quantifications for $\mathcal{S}_{1}^{R}, \ldots, \mathcal{S}_{n_{f}}^{R}$, and for each tuple of pseudometric symbols $d_{1}, \ldots, d_{n_{f}}$ of sort $\mathcal{S}_{1}, \ldots, \mathcal{S}_{n_{f}}$, a distinguished compact interval $D_{D_{1}, \ldots, D_{n_{R}}}^{R}$ in $\mathbb{R}$ and a distinguished continuity modulus $\varpi_{D_{1}, \ldots, D_{n}}^{R}$.

A function symbol $f$ is allowed to have arity $n_{f}=0$, in which case it is called a constant symbol. Given a language $\mathcal{L}$, one can define the notion of $\mathcal{L}$-term by recursion. Formally, one declares that variables are terms, and if $t_{1}, \ldots, t_{n}$ are $\mathcal{L}$-terms and $f$ is an $n$-ary function symbol in $\mathcal{L}$, then $f\left(t_{1}, \ldots, t_{n}\right)$ is an $\mathcal{L}$-term. If $t$ is an $\mathcal{L}$-term and $x_{1}, \ldots, x_{k}$ are the variables that appear in $t$, then one also writes $t$ as $t\left(x_{1}, \ldots, x_{k}\right)$. 
Formulas are defined starting from terms by recursion. A basic $\mathcal{L}$-formula in the free variables $\bar{x}=\left(x_{1}, \ldots, x_{k}\right)$ is an expression $\varphi(\bar{x})$ of the form $R\left(t_{1}, \ldots, t_{n}\right)$ where $t_{1}(\bar{x}), \ldots, t_{n}(\bar{x})$ are $\mathcal{L}$-terms and $R$ is an $n$-ary relation symbol in $\mathcal{L}$. A quantifier-free $\mathcal{L}$-formula is an expression $\varphi(\bar{x})$ of the form

$$
q\left(\psi_{1}(\bar{x}), \ldots, \psi_{n}(\bar{x})\right)
$$

where $\psi_{1}, \ldots, \psi_{n}$ are basic $\mathcal{L}$-formulas in the free variables $\bar{x}$ and $q: \mathbb{R}^{n} \rightarrow \mathbb{R}$ is a continuous function. Such a quantifier-free formula is positive if $q: \mathbb{R}^{n} \rightarrow \mathbb{R}$ has the property that $s_{i} \leq t_{i}$ for $i=1,2, \ldots, n$ implies that $q\left(s_{1}, \ldots, s_{n}\right) \leq q\left(t_{1}, \ldots, t_{n}\right)$. If furthermore $q$ is of the form $q\left(z_{1}, \ldots, z_{n}\right)=\max \left\{u_{1}\left(z_{1}\right), \ldots, u_{n}\left(z_{n}\right)\right\}$ where $u_{1}, \ldots, u_{n}: \mathbb{R} \rightarrow \mathbb{R}$ are continuous nondecreasing functions, then we say that $\varphi(\bar{x})$ is a positive primitive quantifier-free $\mathcal{L}$-formula.

A (positive/positive primitive) existential $\mathcal{L}$-formula in the free variables $x_{1}, \ldots, x_{k}$ is an expression of the form $\varphi\left(x_{1}, \ldots, x_{k}\right)=\inf _{y_{1}} \ldots \inf _{y_{\ell}} \psi\left(x_{1}, \ldots, x_{k}, y_{1}, \ldots, y_{\ell}\right)$ where $\psi(\bar{x}, \bar{y})$ is a (positive/positive primitive) quantifier-free $\mathcal{L}$-formula. A (positive/positive primitive) $\forall \exists$ - $\mathcal{L}$-formula in the free variables $x_{1}, \ldots, x_{k}$ is an expression $\varphi\left(x_{1}, \ldots, x_{k}\right)$ of the form $\sup _{z_{1}} \cdots \sup _{z_{m}} \inf _{y_{1}} \cdots \inf _{y_{\ell}} \psi\left(x_{1}, \ldots, x_{k}, y_{1}, \ldots, y_{\ell}, z_{1}, \ldots, z_{m}\right)$ where $\psi(\bar{x}, \bar{y})$ is a (positive/positive primitive) quantifier-free $\mathcal{L}$-formula. Finally, arbitrary (positive/positive primitive) $\mathcal{L}$ formulas are defined similarly as above, but allowing an arbitrary finite number of alternations between sup and inf. A (positive/positive primitive) $\mathcal{L}$-sentence is a (positive/positive primitive) $\mathcal{L}$-formula with no free variables.

For each $\mathcal{L}$-formula $\varphi\left(x_{1}, \ldots, x_{k}\right)$, for each choice $D_{1}, \ldots, D_{k}$ of domains of quantification for the sorts of $x_{1}, \ldots, x_{k}$, and for each choice of pseudometric symbols $d_{1}, \ldots, d_{k}$ for the sorts $x_{1}, \ldots, x_{k}$ one can define by recursion on the complexity of the formula, a uniform continuity modulus $\varpi_{D_{1}, \ldots, D_{k}, d_{1}, \ldots, d_{k}}^{\varphi}$ for $\varphi$, as well as a compact interval $D_{D_{1}, \ldots, D_{k}}^{\varphi}$ in $\mathbb{R}$ where $\varphi$ takes values. A collection $\mathcal{F}$ of formulas in the free variables $x_{1}, \ldots, x_{k}$ is uniformly equicontinuous if for any choice $D_{1}, \ldots, D_{k}$ of domains of quantification for the sorts of $x_{1}, \ldots, x_{k}$, the continuity moduli $\left\{\varpi_{D_{1}, \ldots, D_{k}, d_{1}, \ldots, d_{k}}^{\varphi}: \varphi \in \mathcal{F}\right\}$ are uniformly bounded, and the compact intervals $\left\{D_{D_{1}, \ldots, D_{k}}^{\varphi}: \varphi \in \mathcal{F}\right\}$ are all contains in a common compact interval in $\mathbb{R}$.

A.2. Semantics. An $\mathcal{L}$-structure $M$ if given by:

- for each sort $\mathcal{S}$, a set $\mathcal{S}^{M}$,

- for each sort $\mathcal{S}$, and for each pseudometric symbol $d_{\mathcal{S}}$ of sort $\mathcal{S}, d_{\mathcal{S}}^{M}$ is a pseudometric on $\mathcal{S}$, such that the uniformity defined by these pseudometrics [44, Definition 2.1] is complete [44, Definition 1.14], and such that the assignment $d_{\mathcal{S}} \mapsto d_{\mathcal{S}}^{M}$ is order preserving (where the order for pseudometric is pointwise comparison);

- for each domain of quantification $D$ for $\mathcal{S}$, a closed subset $D^{M}$ of $\mathcal{S}^{M}$ such that the assignment $D \mapsto D^{M}$ is order preserving (where the collection of closed subsets of $\mathcal{S}^{M}$ is ordered with respect to inclusion), and such that the union of $D^{M}$ when $D$ ranges among the domains of quantification for $\mathcal{S}$ is dense in $\mathcal{S}^{M}$ with respect to the uniformity described above,

- for each function symbol $f$ with input sorts $\mathcal{S}_{1}^{f}, \ldots, \mathcal{S}_{n_{f}}^{f}$ and output sort $\mathcal{S}^{f}$, a function $f^{M}:\left(\mathcal{S}_{1}^{f}\right)^{M} \times \cdots \times$ $\left(\mathcal{S}_{n_{f}}^{f}\right)^{M} \rightarrow\left(\mathcal{S}^{f}\right)^{M}$ (the interpretation of $f$ in $M$ ) such that, for any choice of domains of quantification $D_{1}, \ldots, D_{n_{f}}$ and pseudometric symbols $d_{1}, \ldots, d_{n_{f}}$ for the sorts $\mathcal{S}_{1}^{f}, \ldots, \mathcal{S}_{n_{f}}^{f}$, the restriction of $f^{M}$ to $D_{1}^{M} \times \cdots \times D_{n_{f}}^{M}$ is a uniformly continuous map with continuity modulus $\varpi_{D_{1}, \ldots, D_{n_{f}}, d_{1}, \ldots, d_{n_{f}}}^{f}$ with respect to the metrics $\max \left\{d_{1}, \ldots, d_{n_{f}}\right\}$ and $d_{D_{1}, \ldots, D_{n_{f}}, d_{1}, \ldots, d_{n_{f}}}^{f}$, and its range is contained in $\left(D_{D_{1}, \ldots, D_{n_{f}}}^{f}\right)^{M}$;

- for each relation symbol $R$ with input sorts $\mathcal{S}_{1}^{R}, \ldots, \mathcal{S}_{n_{f}}^{R}$ and output sort $\mathcal{S}^{R}$, a function $R^{M}:\left(\mathcal{S}_{1}^{R}\right)^{M} \times$ $\cdots \times\left(\mathcal{S}_{n_{R}}^{R}\right)^{M} \rightarrow \mathbb{R}$ (the interpretation of $R$ in $M$ ) such that, for any choice of domains of quantification $D_{1}, \ldots, D_{n_{f}}$ and pseudometric symbols $d_{1}, \ldots, d_{n_{f}}$ for $\mathcal{S}_{1}^{R}, \ldots, \mathcal{S}_{n_{R}}^{R}$, the restriction of $f^{M}$ to $D_{1}^{M} \times$ $\cdots \times D_{n_{f}}^{M}$ is a uniformly continuous map with continuity modulus $\varpi_{D_{1}, \ldots, D_{n_{f}}, d_{1}, \ldots, d_{n_{f}}}^{R}$ and its range is contained in $D_{D_{1}, \ldots, D_{n_{R}}}^{R}$.

Suppose now that $t\left(x_{1}, \ldots, x_{k}\right)$ is an $\mathcal{L}$-term in the variables $x_{1}, \ldots, x_{k}$ of sorts $\mathcal{S}_{1}, \ldots, \mathcal{S}_{k}$, and that $M$ is an $\mathcal{L}$-structure. Then one can define by recursion on the complexity of $t$ the output sort $\mathcal{S}$ of $t$ and the interpretation $t^{M}$ of $t$ in $M$, which is a function $t^{M}: \mathcal{S}_{1}^{M} \times \cdots \times \mathcal{S}_{k}^{M} \rightarrow \mathcal{S}^{M}$. Similarly, for any $\mathcal{L}$-formula $\varphi\left(x_{1}, \ldots, x_{k}\right)$ in the free variables $x_{1}, \ldots, x_{k}$ of sorts $\mathcal{S}_{1}, \ldots, \mathcal{S}_{k}$, one can defined by recursion on the complexity of $\varphi$ its interpretation $\varphi^{M}$ in $M$, which is a function $\varphi^{M}: \mathcal{S}_{1}^{M} \times \cdots \times \mathcal{S}_{k}^{M} \rightarrow \mathbb{R}$. For any choice of domains of quantification $D_{1}, \ldots, D_{k}$ for the sorts of $x_{1}, \ldots, x_{k}$, the restriction of $\varphi^{M}$ to $D_{1}^{M} \times \cdots \times D_{k}^{M}$ is uniformly 
continuous with the continuity modulus $\varpi_{D_{1}, \ldots, D_{k}}^{\varphi}$ as in the previous section, and takes values in the compact interval $D_{D_{1}, \ldots, D_{k}}^{\varphi}$ of $\mathbb{R}$.

Suppose now that $\mathcal{C}$ is a class of $\mathcal{L}$-structures. Then $\mathcal{C}$ defines a metric in the class of $\mathcal{L}$-formulas by setting, for every $\mathcal{L}$-formulas $\varphi\left(x_{1}, \ldots, x_{k}\right), \psi\left(x_{1}, \ldots, x_{k}\right)$,

$$
d_{\mathcal{C}}(\varphi, \psi)=\sup |\varphi(\bar{a})-\psi(\bar{a})|
$$

where $M$ ranges among all the $\mathcal{L}$-structures in $\mathcal{C}$, and $\bar{a}$ ranges among all the tuples in $M$ of the correct sorts.

Definition A.1. The language $\mathcal{L}$ is separable for a class of $\mathcal{L}$-structures $\mathcal{C}$ if the space of $\mathcal{L}$-formulas endowed with the metric associated with $\mathcal{C}$ as above is separable. More generally, we define the density character of $\mathcal{L}$ for $\mathcal{C}$ to be the density character of the space of $\mathcal{L}$-formulas with respect to the metric $d_{\mathcal{C}}$.

Definition A.2. A class $\mathcal{C}$ of $\mathcal{L}$-structures is axiomatizable in the language $\mathcal{L}$ if there exists a collection $\mathcal{A}$ of $\mathcal{L}$-conditions of the form $\sigma \leq r$ (axioms) where $\sigma$ is an $\mathcal{L}$-sentence and $r \in \mathbb{R}$, such that for any $\mathcal{L}$-structure $M$, $M$ belongs to $\mathcal{C}$ if and only if $\sigma^{M} \leq r$ for any condition in $\mathcal{A}$.

Definition A.3. A class of $\mathcal{L}$-structures is (positively/positively primitively) $\forall \exists$-axiomatizable in the language $\mathcal{L}$ if it is axiomatizable by a collection of $\mathcal{L}$-conditions of the form $\sigma \leq r$ where $\sigma$ is a (positive/positive primitive) $\forall \exists$ - $\mathcal{L}$-sentence and $r \in \mathbb{R}$.

The following notion has been essentially introduced in [12, Definition 5.7.1].

Definition A.4. A class $\mathcal{C}$ of $\mathcal{L}$-structures is definable by a uniform collection of positive existential $\mathcal{L}$-formulas if for every choice of sorts $\mathcal{S}_{1}, \ldots, \mathcal{S}_{k}$ in $\mathcal{L}$ and domains of quantification $D_{1}, \ldots, D_{k}$ for $\mathcal{S}_{1}, \ldots, \mathcal{S}_{k}$, there exist and a uniformly equicontinuous family $\mathcal{F}\left(D_{1}, \ldots, D_{k}\right)$ of positive existential $\mathcal{L}$-formulas in the free variables $x_{1}, \ldots, x_{k}$ of sorts $\mathcal{S}_{1}, \ldots, \mathcal{S}_{k}$ such that, for every $\mathcal{L}$-structure $M$, the following assertions are equivalent:

(1) $M$ belongs to $\mathcal{C}$;

(2) for every choice of sorts $\mathcal{S}_{1}, \ldots, \mathcal{S}_{k}$ in $\mathcal{L}$, of domains of quantification $D_{1}, \ldots, D_{k}$ for $\mathcal{S}_{1}, \ldots, \mathcal{S}_{k}$, of elements $a_{i} \in D_{i}^{M}$ for $i=1,2, \ldots, k$, and for every $\varepsilon>0$, there exists a formula $\varphi\left(x_{1}, \ldots, x_{k}\right)$ in $\mathcal{F}\left(D_{1}, \ldots, D_{k}\right)$ such that $M \models \varphi\left(a_{1}, \ldots, a_{k}\right) \leq \varepsilon$.

It is clear that, if a class of $\mathcal{L}$-structures is positively $\forall \exists$-axiomatizable in the language $\mathcal{L}$, then in particular it is definable by a uniform collection of positive existential $\mathcal{L}$-formulas.

A.3. Reduced products and ultraproducts. Reduced products and ultraproducts are a fundamental construction in model theory. We recall here these notions and their basic properties. Suppose that $\mathcal{L}$ is a language as above, $I$ is an index set, $M_{i}$ for $i \in I$ is an $\mathcal{L}$-structure, and $\mathcal{F}$ is a filter over $I$. One can define the reduced $\mathcal{L}$-product $\prod_{\mathcal{F}} M_{i}$ of the $I$-sequence $\left(M_{i}\right)_{i \in I}$ with respect to $\mathcal{F}$ as follows. For each sort $\mathcal{S}$ in $\mathcal{L}$ and domain of quantification $D$ for $\mathcal{S}$, and for each pseudometric symbol $d$ of sort $\mathcal{S}$, consider the pseudometric $d^{M}$ on $\prod_{i} D_{i}^{M}$ defined by $d\left(\left(x_{i}\right),\left(y_{i}\right)\right)=\lim \sup _{\mathcal{F}} d^{M_{i}}\left(x_{i}, y_{i}\right)$. The collection of such pseudometrics, when $d$ ranges among the pseudometric symbols of sort $\mathcal{S}$, defines a uniformity on $\prod_{i} D_{i}^{M}$, and then one can define $D^{M}$ to be the Hausdorff completion of such a uniform space, endowed with the canonical pseudometrics induces by the pseudometrics on $\prod_{i} D_{i}^{M}$. We will denote by $\left[x_{i}\right]_{\mathcal{F}}$ the element of $D^{M}$ associated with the sequence $\left(x_{i}\right)_{i \in I}$, and we will call $\left(x_{i}\right)$ a representative sequence for $\left[x_{i}\right]_{\mathcal{F}}$.

If $D_{1}, D_{2}$ are domains of quantification for $\mathcal{S}$ with $D_{1} \leq D_{2}$, then one can canonically identify $D_{1}^{M}$ as a closed subspace of $D_{2}^{M}$. Since the collection of domains of quantification for $\mathcal{S}$ is directed, the union of $D^{M}$, where $D$ ranges among all the domains of quantifications for $\mathcal{S}$, is a (possibly incomplete) metric space. Define then $\mathcal{S}^{M}$ to be the completion of such a metric space. One can regard $D^{M}$ as a closed subspace of $\mathcal{S}^{M}$. Clearly, by definition of $\mathcal{S}^{M}$, the union of $D^{M}$, where $D$ ranges among all the quantifications for $\mathcal{S}$, is dense in $\mathcal{S}^{M}$.

For any function symbol $f$ in $\mathcal{L}$ with input sorts $\mathcal{S}_{1}^{f}, \ldots, \mathcal{S}_{n_{f}}^{f}$ and output sort $\mathcal{S}^{f}$, one can then define a function $f^{M}:\left(\mathcal{S}_{1}^{f}\right)^{M} \times \cdots \times\left(\mathcal{S}_{n_{f}}^{f}\right)^{M} \rightarrow\left(\mathcal{S}^{f}\right)^{M}$ by setting, for every choice of domains of quantification $D_{1}, \ldots, D_{n}$ for $\mathcal{S}_{1}^{f}, \ldots, \mathcal{S}_{n_{f}}^{f}$, and elements $\left[a_{i}^{1}\right]_{\mathcal{F}}, \ldots,\left[a_{i}^{n}\right]_{\mathcal{F}}$ of $D_{1}^{M}, \ldots, D_{n}^{M}$,

$$
f\left(\left[a_{i}^{1}\right]_{\mathcal{F}}, \ldots,\left[x_{i}^{n}\right]_{\mathcal{F}}\right)=\left[f\left(a_{i}^{1}, \ldots, a_{i}^{n}\right)\right]_{\mathcal{F}} \in\left(D_{D_{1}, \ldots, D_{n}}^{f}\right)^{M} .
$$

Similarly, for any relation symbol $R$ in $\mathcal{L}$ with input sorts $\mathcal{S}_{1}^{R}, \ldots, \mathcal{S}_{n_{f}}^{R}$ and output sort $\mathcal{S}^{R}$, one can define a function $R^{M}:\left(\mathcal{S}_{1}^{R}\right)^{M} \times \cdots \times\left(\mathcal{S}_{n_{R}}^{R}\right)^{M} \rightarrow \mathbb{R}$ by setting, for every choice of domains of quantification $D_{1}, \ldots, D_{n}$ for $\mathcal{S}_{1}^{R}, \ldots, \mathcal{S}_{n_{R}}^{R}$, and elements $\left[a_{i}^{1}\right]_{\mathcal{F}}, \ldots,\left[a_{i}^{n}\right]_{\mathcal{F}}$ of $D_{1}^{M}, \ldots, D_{n}^{M}$,

$$
R\left(\left[a_{i}^{1}\right]_{\mathcal{F}}, \ldots,\left[a_{i}^{n}\right]_{\mathcal{F}}\right)=\limsup _{\mathcal{F}} R\left(a_{i}^{1}, \ldots, a_{i}^{n}\right) .
$$


One obtains in this way an $\mathcal{L}$-structure $M$, which is called the reduced $\mathcal{L}$-product of the $I$-sequence of $\mathcal{L}$ structures $\left(M_{i}\right)_{i \in I}$ with respect to the filter $\mathcal{F}$, and denoted by $\prod_{\mathcal{F}} M_{i}$. In the case when $\mathcal{F}$ is an ultrafilter, then $\prod_{\mathcal{F}} M_{i}$ is called $\mathcal{L}$-ultraproduct of the $I$-sequence $\left(M_{i}\right)_{i \in I}$. The function that maps an element $a$ of $M$ to the element of $\prod_{\mathcal{F}} M_{i}$ with representative sequence constantly equal to $a$ defines the canonical diagonal $\mathcal{L}$-embedding of $M$ into $\prod_{\mathcal{F}} M_{i}$. The fundamental properly of reduced $\mathcal{L}$-products is the following result, known as Los' theorem; see [15, Proposition 4.3].

Proposition A.5. Suppose that $\mathcal{F}$ is a filter on a set $I,\left(M_{i}\right)_{i \in I}$ is an I-sequence of $\mathcal{L}$-structures, and $\varphi\left(x_{1}, \ldots, x_{k}\right)$ is a formula in the free variables $x_{1}, \ldots, x_{n}$. If either $\varphi$ is positive primitive quantifier-free, or $\mathcal{F}$ is an ultrafilter, then for any $\left[a_{i}^{1}\right], \ldots,\left[a_{i}^{k}\right]$ in $\prod_{\mathcal{F}} M_{i}$ one has

$$
\varphi^{\prod_{\mathcal{F}} M_{i}}\left(\left[a_{i}^{1}\right], \ldots,\left[a_{i}^{k}\right]\right)=\limsup _{\mathcal{F}} \varphi^{M_{i}}\left(a_{i}^{1}, \ldots, a_{i}^{k}\right) .
$$

Corollary A.6. Suppose that $\mathcal{C}$ is a class of $\mathcal{L}$-structures that is axiomatizable in the language $\mathcal{L}$. Then $\mathcal{C}$ is closed under $\mathcal{L}$-ultraproducts. If $\mathcal{C}$ is furthermore positively primitively $\forall \exists$-axiomatizable in the language $\mathcal{L}$, then $\mathcal{C}$ is closed under reduced $\mathcal{L}$-products.

A.4. Types and saturation. A (closed) $\mathcal{L}$-condition is an expression of the form $\varphi(\bar{x}) \leq r$ for some $\mathcal{L}$-formula $\varphi$ in the free variables $\bar{x}=\left(x_{1}, \ldots, x_{k}\right)$ and $r \in \mathbb{R}$. An $\mathcal{L}$-type $p\left(x_{1}, \ldots, x_{k}\right)$ in the free variables $x_{1}, \ldots, x_{k}$ is a collection of $\mathcal{L}$-conditions $\varphi(\bar{x}) \leq r$. Such a type $p$ is quantifier-free if all the conditions that appear in it involve quantifier-free $\mathcal{L}$-formulas, and positive quantifier-free if all the conditions that appear in it involve positive primitive quantifier-free $\mathcal{L}$-formulas. A realization of the $\mathcal{L}$-type $p(\bar{x})$ in an $\mathcal{L}$-structure $M$ is a tuple $\bar{a}=\left(a_{1}, \ldots, a_{k}\right)$ in $M$ such that each $a_{i}$ belongs to the interpretation of the sort of $x_{i}$, and $\varphi^{M}(\bar{a}) \leq r$ for every condition $\varphi(\bar{x}) \leq r$ in $p(\bar{x})$. In this case, we also write $M \models \varphi(\bar{a}) \leq r$ and $M \models p(\bar{a})$. An $\mathcal{L}$-type is realized in an $\mathcal{L}$-structure $M$ if it admits a realization in $M$. It is approximately realized in $M$ if for any finite subset $p_{0}(\bar{x})$ of $p(\bar{x})$ and for any $\varepsilon>0$, the type $p_{0}^{\varepsilon}\left(x_{1}, \ldots, x_{k}\right)$ consisting of the conditions $\varphi(\bar{x}) \leq r+\varepsilon$ for any condition $\varphi(\bar{x}) \leq r$ in $p_{0}\left(x_{1}, \ldots, x_{k}\right)$, is realized in $M$.

Suppose that $M$ is an $\mathcal{L}$-structure, and $A$ is a subset of $M$. Then one can consider the language $\mathcal{L}(A)$ obtained by adding to $\mathcal{L}$ a constant symbol for each element of $A$. Then $M$ or any $\mathcal{L}$-structure containing $M$ can be canonically regarded as an $\mathcal{L}(A)$-structure.

Definition A.7. Suppose that $\kappa$ is an uncountable cardinal. An $\mathcal{L}$-structure $M$ is $\mathcal{L}$ - $\kappa$-saturated if for every subset $A$ of $M$ of density character less than $\kappa$ and for any $\mathcal{L}(A)$-type $p$, if $p$ is approximately realized in $M$, then it is realized in $M$. Replacing arbitrary types with positive quantifier-free types gives the notion of positively quantifier-free $\mathcal{L}$ - $\kappa$-saturated structure.

In the particular case when $\kappa=\aleph_{1}, \mathcal{L}$ - $\aleph_{1}$-saturation is also called countable $\mathcal{L}$-saturation, and positive quantifier-free $\mathcal{L}$ - $\kappa$-saturation is also called positive quantifier-free countable $\mathcal{L}$-saturation. The fundamental property of ultrapowers of structures with respect to nonprincipal ultrafilters over $\mathbb{N}$ is that they are countably saturated; see [15, Proposition 4.11].

Proposition A.8. Suppose that $\mathcal{F}$ is a countably incomplete filter. Let $\mathcal{C}$ be a class of $\mathcal{L}$-structures such that $\mathcal{L}$ is separable for $\mathcal{C}$. If $M$ is an $\mathcal{L}$-structure in $\mathcal{C}$, then the reduced power $\prod_{\mathcal{F}} M$ is countably positively quantifier-free $\mathcal{L}$-saturated. If $\mathcal{F}$ is a countably incomplete ultrafilter, then the ultrapower $\prod_{\mathcal{U}} M$ is countably $\mathcal{L}$-saturated.

Proposition A.8 admits a generalization to an arbitrary uncountable cardinal $\kappa$. In this more general setting, one needs to consider (ultra)filters that are moreover $\kappa$-good. The definition of $\kappa$-good is given in [5, Section 6.1] for ultrafilters, but it applies equally well to filters. Every countably incomplete filter is $\aleph_{1}$-good. In particular, every nonprincipal ultrafilter over $\mathbb{N}$ is $\aleph_{1}$-good. The same proof as [5, Theorem 6.1.8] gives the following.

Proposition A.9. Let $\kappa$ be an uncountable cardinal, and let $\mathcal{F}$ be a countably incomplete $\kappa$-good filter. Let $\mathcal{C}$ be a class of $\mathcal{L}$-structures such that $\mathcal{L}$ has density character less than $\kappa$ for $\mathcal{C}$. If $M$ is an $\mathcal{L}$-structure, then the reduced power $\prod_{\mathcal{F}} M$ is positively quantifier-free $\mathcal{L}$ - $\kappa$-saturated. If $\mathcal{U}$ is a countably incomplete $\kappa$-good ultrafilter, then the ultrapower $\prod_{\mathcal{U}} M$ is $\mathcal{L}$ - $\kappa$-saturated.

A.5. Existential embeddings. Suppose that $\mathcal{L}$ is a language in the logic for metric structures as above, $M, N$ are $\mathcal{L}$-structures, and $T: M \rightarrow N$ is a function. Then $T$ is an $\mathcal{L}$-morphism if, for every sort $\mathcal{S}$ in $\mathcal{L}$ and for every domain of quantification $D$ for $\mathcal{S}, T$ maps $\mathcal{S}^{M}$ to $\mathcal{S}^{N}$ and $D^{M}$ to $D^{N}$, and for any atomic formula $\varphi\left(x_{1}, \ldots, x_{k}\right)$ one has that $\varphi^{N}\left(T\left(a_{1}\right), \ldots, T\left(a_{k}\right)\right) \leq \varphi^{M}\left(a_{1}, \ldots, a_{k}\right)$ for any $a_{1}, \ldots, a_{n}$ of the same sorts as $x_{1}, \ldots, x_{k}$. An $\mathcal{L}$-morphism is an $\mathcal{L}$-embedding if for any atomic formula $\varphi\left(x_{1}, \ldots, x_{k}\right)$ one has that $\varphi^{N}\left(T\left(a_{1}\right), \ldots, T\left(a_{k}\right)\right)=$ $\varphi^{M}\left(a_{1}, \ldots, a_{k}\right)$ for any $a_{1}, \ldots, a_{n}$ of the same sorts as $x_{1}, \ldots, x_{k}$. Suppose that $T: M \rightarrow N$ is an $\mathcal{L}$-embedding, 
and $A$ is a subset of $M$. Recall that $\mathcal{L}(A)$ is the language obtained from $\mathcal{L}$ by adding a constant symbols $c_{a}$ for any element $a$ of $A$. Then one can regard both $M$ and $N$ as $\mathcal{L}(A)$-structures, by interpreting $c_{a}$ as $a$ in $M$ and as $T(a)$ in $N$. We recall here the notion of (positively) $\mathcal{L}$-existential $\mathcal{L}$-embedding.

Definition A.10. Suppose that $M, N$ are $\mathcal{L}$-structures, and $T: M \rightarrow N$ is an $\mathcal{L}$-embedding. Then $T$ is a (positively) $\mathcal{L}$-existential if for every (positive) quantifier-free $\mathcal{L}(M)$-condition $\varphi\left(x_{1}, \ldots, x_{n}\right) \leq r$ satisfied in $N$ and for every $\varepsilon>0$, then $\mathcal{L}(M)$-condition $\varphi\left(x_{1}, \ldots, x_{n}\right) \leq r+\varepsilon$ is satisfied in $M$.

The following characterization of $\mathcal{L}$-existential $\mathcal{L}$-embeddings is an immediate consequence of Eos' theorem and saturation of reduced powers. Recall that any countably incomplete filter is $\aleph_{1}$-good.

Proposition A.11. Fix an uncountable cardinal $\kappa$, a class $\mathcal{C}$ of $\mathcal{L}$-structures, and structures $M, N$ in $\mathcal{C}$ of density character less than $\kappa$. Suppose that $\mathcal{F}$ is a countably incomplete $\kappa$-good filter. Let $T: M \rightarrow N$ be an $\mathcal{L}$-morphism. The following assertions are equivalent:

(1) $T$ is a positively $\mathcal{L}$-existential $\mathcal{L}$-embedding;

(2) there exists an $\mathcal{L}$-morphism $S: N \rightarrow \prod_{\mathcal{F}} M$ such that $S \circ T$ is the diagonal $\mathcal{L}$-embedding $M \rightarrow \prod_{\mathcal{F}} M$.

Furthermore, if $\mathcal{F}$ is an ultrafilter, then the following assertions are equivalent:

(1) $T$ is an $\mathcal{L}$-existential $\mathcal{L}$-embedding;

(2) there exists an $\mathcal{L}$-embedding $S: N \rightarrow \prod_{\mathcal{U}} M$ such that $S \circ T$ is the diagonal $\mathcal{L}$-embedding $M \rightarrow \prod_{\mathcal{U}} M$.

The following preservation result for positively $\mathcal{L}$-existential $\mathcal{L}$-embedding can be easily verified directly.

Proposition A.12. Suppose that $\mathcal{C}$ is a collection of $\mathcal{L}$-structures that is definable by a uniform collection of positive existential $\mathcal{L}$-formulas. Suppose that $A$ and $B$ are $\mathcal{L}$-structures, and $T: A \rightarrow B$ is a positively $\mathcal{L}$-existential $\mathcal{L}$-embedding. If $B$ belongs to $\mathcal{C}$, then $A$ belongs to $\mathcal{C}$.

A.6. Definability. Suppose that $\mathcal{C}$ is a class of $\mathcal{L}$-structures. Fix domains of quantifications $D_{1}, \ldots, D_{n}$ for $\mathcal{L}$ of sorts $\mathcal{S}_{1}, \ldots, \mathcal{S}_{n}$. A uniform assignment $S$ in $D_{1} \times \cdots \times D_{n}$ relative to the class $\mathcal{C}$ is an assignment $M \mapsto S^{M}$, where $M$ ranges among the $\mathcal{L}$-structures in $\mathcal{C}$ and $S^{M}$ is a closed subset $D_{1}^{M} \times \cdots \times D_{k}^{M}$; see [12, Definition 3.2.1]. The following definition is equivalent to the one given in [12, Definition 3.2.1]; see [12, Theorem 3.2.2].

Definition A.13. Suppose that $S$ is a uniform assignment in $D_{1} \times \cdots \times D_{n}$ relative to the class $\mathcal{C}$. Then $S$ is an $\mathcal{L}$-definable set in $D_{1} \times \cdots \times D_{n}$ relative to the class $\mathcal{C}$ if for every $\varepsilon>0$, for every choice of pseudometric symbols $d_{1}, \ldots, d_{n}$ of sorts $\mathcal{S}_{1}, \ldots, \mathcal{S}_{n}$ there exists an $\mathcal{L}$-formula $\varphi\left(x_{1}, \ldots, x_{k}\right)$ in the free variables $x_{1}, \ldots, x_{n}$ of sorts $\mathcal{S}_{1}, \ldots, \mathcal{S}_{n}$ such that, for every $\mathcal{L}$-structure $M$ in $\mathcal{C}$ and for every $\left(a_{1}, \ldots, a_{n}\right) \in D_{1} \times \cdots \times D_{n}$,

$$
\left|\varphi^{M}\left(a_{1}, \ldots, a_{n}\right)-\inf _{\left(b_{1}, \ldots, b_{n}\right) \in S^{M}} \max _{1 \leq i \leq n} d_{i}\left(a_{i}, b_{i}\right)\right|<\varepsilon .
$$

We say that $S$ is positively existentially $\mathcal{L}$-definable in the $\mathcal{L}$-formulas $\varphi$ above can be chosen to be positive existential.

Observe that, every choice of pseudometric symbols $d_{1}, \ldots, d_{n}$ of sort $\mathcal{S}_{1}, \ldots, \mathcal{S}_{n}$ defines a pseudometric $\rho$ on the space of $\mathcal{L}$-definable sets in $D_{1} \times \cdots \times D_{n}$ relative to the class $\mathcal{C}$, obtained by setting

$$
\rho\left(S, S^{\prime}\right)=\sup _{M \in \mathcal{C}} \rho^{M}\left(S^{M}, S^{M}\right),
$$

where $\rho^{M}$ is the Hausdorff metric on the space of closed subsets of $D_{1}^{M} \times \cdots \times D_{n}^{M}$ associated with the metric $d(\bar{a}, \bar{b})=\max _{1 \leq i \leq n} d_{i}\left(a_{i}, b_{i}\right)$ on $D_{1}^{M} \times \cdots \times D_{n}^{M}$. The collection of such pseudometrics $\rho$, obtaining by letting $d_{1}, \ldots, d_{n}$ range among all the pseudometric symbols of sort $\mathcal{S}_{1}, \ldots, \mathcal{S}_{n}$, define a uniform structure on the space of $\mathcal{L}$-definable sets in $D_{1} \times \cdots \times D_{n}$ relative to the class $\mathcal{C}$. It is clear from the definition that the space of (positively existentially) $\mathcal{L}$-definable sets is complete with respect to such a uniform structure.

Suppose now that $M$ is an $\mathcal{L}$-structure, and $\mathcal{C}$ is a class of $\mathcal{L}$-structures as above. Then one can consider the class $\mathcal{C}(M)$ of $\mathcal{L}$-structures in $\mathcal{C}$ that contain a distinguished copy of $M$. Then one can naturally regard the structures in $\mathcal{C}(M)$ as $\mathcal{L}(M)$-structures. We consider a natural notion of $\mathcal{L}$-definable substructure. Suppose as above that $\mathcal{C}$ is a class of $\mathcal{L}$-structures.

Definition A.14. A positively existentially $\mathcal{L}$-definable substructure relative to the class $\mathcal{C}$ is an assignment $(M, D) \mapsto S^{D, M}$ where $M$ ranges among the $\mathcal{L}$-structures in $\mathcal{C}$ and $D$ ranges among the domains of quantification in $\mathcal{L}$ such that:

- $S^{D, M}$ is a closed subset of $D^{M}$, 
- setting $S(M)^{D}:=S^{D, M}$ for every sort $\mathcal{S}$ and every domain of quantification $D$ of sort $\mathcal{S}$ defines an $\mathcal{L}$-substructure $S(M)$ of $M$,

- for every domain $D$, the assignment $M \mapsto S^{D, M}$ is a positively existentially $\mathcal{L}$-definable set in $D$ relative to the class $\mathcal{C}$.

The following proposition is an easy consequence of the definition of positive existential $\mathcal{L}$-embedding.

Proposition A.15. Suppose that $M, N$ are $\mathcal{L}$-structures, and $T: M \rightarrow N$ is a positively $\mathcal{L}$-existential $\mathcal{L}$ embedding. Suppose that $S$ is a positively existentially $\mathcal{L}(M)$-definable substructure relative to a class $\mathcal{C}$ of $\mathcal{L}(M)$-structures containing $M$ and $N$. Then $T$ maps $S(M)$ to $S(N)$, and the restriction $\left.T\right|_{S(M)}: S(M) \rightarrow S(N)$ is a positively $\mathcal{L}$-existential $\mathcal{L}$-embedding.

\section{REFERENCES}

[1] Selçuk Barlak and Gábor Szabó, Sequentially split *-homomorphisms between $C^{*}$-algebras, International Journal of Mathematics 27 (2016), no. 13.

[2] Selçuk Barlak, Gábor Szabó, and Christian Voigt, The spatial Rokhlin property for actions of compact quantum groups, Journal of Functional Analysis 272 (2017), no. 6, 2308-2360.

[3] Itaï Ben Yaacov, Alexander Berenstein, C. Ward Henson, and Alexander Usvyatsov, Model theory for metric structures, Model theory with applications to algebra and analysis. Vol. 2, London Mathematical Society Lecture Note Series, vol. 350, Cambridge University Press, 2008, pp. 315-427.

[4] Kevin Carlson, Enoch Cheung, Ilijas Farah, Alexander Gerhardt-Bourke, Bradd Hart, Leanne Mezuman, Nigel Sequeira, and Alexander Sherman, Omitting types and AF algebras, Archive for Mathematical Logic 53 (2014), no. 1-2, 157-169.

[5] Chen C. Chang and H. Jerome Keisler, Model theory, second ed., North-Holland Publishing Co., Amsterdam-New York-Oxford, 1977, Studies in Logic and the Foundations of Mathematics, 73.

[6] Kenny De Commer, Actions of compact quantum groups, arXiv:1604.00159 (2016).

[7] Christopher J. Eagle, Ilijas Farah, Bradd Hart, Boris Kadets, Vladyslav Kalashnyk, and Martino Lupini, Fraïsé limits of $C^{*}$-algebras, Journal of Symbolic Logic 81 (2016), no. 2, 755-773.

[8] Christopher J. Eagle, Isaac Goldbring, and Alessandro Vignati, The pseudoarc is a co-existentially closed continuum, Topology and its Applications 207 (2016), 1-9.

[9] Christopher J. Eagle and Alessandro Vignati, Saturation and elementary equivalence of $C^{*}$-algebras, Journal of Functional Analysis 269 (2015), no. 8, 2631-2664.

[10] David Alexandre Ellwood, A new characterisation of principal actions, Journal of Functional Analysis $\mathbf{1 7 3}$ (2000), no. 1, 49-60.

[11] Ilijas Farah and Bradd Hart, Countable saturation of corona algebras, Comptes Rendus Mathématiques de l'Académie des Sciences 35 (2013), no. 2, 35-56.

[12] Ilijas Farah, Bradd Hart, Martino Lupini, Leonel Robert, Aaron Tikuisis, Alessandro Vignati, and Winter Winter, Model theory of $C^{*}$-algebras, arXiv:1602.08072 (2016).

[13] Ilijas Farah, Bradd Hart, Mikael Rørdam, and Aaron Tikuisis, Relative commutants of strongly selfabsorbing $C^{*}$-algebras, Selecta Mathematica 23 (2017), no. 1, 363-387.

[14] Ilijas Farah, Bradd Hart, and David Sherman, Model theory of operator algebras I: stability, Bulletin of the London Mathematical Society 45 (2013), no. 4, 825-838.

[15] _ Model theory of operator algebras II: model theory, Israel Journal of Mathematics 201 (2014), no. $1,477-505$.

[16] - Model theory of operator algebras III: elementary equivalence and $I I_{1}$ factors, Bulletin of the London Mathematical Society 46 (2014), no. 3, 609-628.

[17] Eusebio Gardella, Crossed products by compact group actions with the Rokhlin property, Journal of Noncommutative Geometry, in press.

[18] _ Regularity properties and Rokhlin dimension for compact group actions, Houston Journal of Mathematics, in press.

[19] _ Rokhlin dimension for compact group actions, Indiana Journal of Mathematics, in press.

[20] Classification theorems for circle actions on Kirchberg algebras, II, arXiv:1406.1208 (2014).

[21] Eusebio Gardella, Ilan Hirshberg, and Luis Santiago, Rokhlin dimension: tracial properties and crossed products, preprint.

[22] Eusebio Gardella and Martino Lupini, Equivariant logic and applications to $C^{*}$-dynamics, arXiv:1608.05532 (2016). 
[23] Saeed Ghasemi, Reduced products of metric structures: a metric Feferman-Vaught theorem, The Journal of Symbolic Logic 81 (2016), no. 3, 856-875.

[24] Isaac Goldbring and Thomas Sinclair, On Kirchberg's embedding problem, Journal of Functional Analysis 269 (2015), no. 1, 155-198.

[25] - On the axiomatizability of $C^{*}$-algebras as operator systems, arXiv:1603.05444 (2016).

[26] Stefan Heinrich, Ultraproducts in Banach space theory, Journal für die Reine und Angewandte Mathematik 313 (1980), 72-104.

[27] Ilan Hirshberg, Gábor Szabó, Wilhelm Winter, and Jianchao Wu, Rokhlin dimension for flows, arXiv:1607.02222 (2016).

[28] Ilan Hirshberg, Wilhelm Winter, and Joachim Zacharias, Rokhlin dimension and $C^{*}$-dynamics, Communications in Mathematical Physics 335 (2015), no. 2, 637-670.

[29] Masaki Izumi, Finite group actions on $C^{*}$-algebras with the Rohlin property, I, Duke Mathematical Journal 122 (2004), no. 2, 233-280.

[30] Eberhard Kirchberg, Central sequences in $C^{*}$-algebras and strongly purely infinite algebras, Operator Algebras: The Abel Symposium 2004, Abel Symp., vol. 1, Springer, Berlin, 2006, pp. 175-231.

[31] Eberhard Kirchberg and Wilhelm Winter, Covering dimension and quasidiagonality, International Journal of Mathematics 15 (2004), no. 01, 63-85.

[32] Akitaka Kishimoto, The Rohlin property for automorphisms of UHF algebras, Journal für die reine und angewandte Mathematik 1995 (1995), no. 465, 183-196.

[33] Kazunori Kodaka and Tamotsu Teruya, The Rohlin property for coactions of finite dimensional $C^{*}$-Hopf algebras on unital $C^{*}$-algebras, Journal of Operator Theory $\mathbf{7 4}$ (2015), no. 2, 329-369.

[34] Johan Kustermans and Lars Tuset, A survey of $C^{*}$-algebraic quantum groups. I, Irish Mathematical Society Bulletin (1999), no. 43, 8-63.

[35] $\longrightarrow$ A survey of $C^{*}$-algebraic quantum groups. II, Irish Mathematical Society Bulletin (2000), no. 44, $6-54$.

[36] Johan Kustermans and Stefaan Vaes, Locally compact quantum groups, Annales Scientifiques de lcole Normale Suprieure 33 (2000), no. 6, 837-934.

[37] - The operator algebra approach to quantum groups, Proceedings of the National Academy of Sciences of the United States of America 97 (2000), no. 2, 547-552 (electronic).

[38] L Locally compact quantum groups in the von Neumann algebraic setting, Mathematica Scandinavica 92 (2003), no. 1, 68-92.

[39] Ann Maes and Alfons Van Daele, Notes on compact quantum groups, Nieuw Archief voor Wiskunde. Vierde Serie 16 (1998), no. 1-2, 73-112.

[40] Shuhei Masumoto, A Fraïsé theoretic approach to the Jiang-Su algebra, arXiv:1612.00646 (2016).

[41] _ Jiang-Su Algebra as a Fraïssé limit, arXiv:1602.00124 (2016).

[42] Norio Nawata, Finite group actions on certain stably projectionless $C^{*}$-algebras with the Rohlin property, Transactions of the American Mathematical Society 368 (2016), no. 1, 471-493.

[43] Ryszard Nest and Christian Voigt, Equivariant Poincaré duality for quantum group actions, Journal of Functional Analysis 258 (2010), no. 5, 1466-1503.

[44] Jan Pachl, Uniform spaces and measures, Fields Institute Monographs, vol. 30, Springer, New York, 2013.

[45] Piotr Podleś, Symmetries of quantum spaces. Subgroups and quotient spaces of quantum $\mathrm{SU}_{2}$ and $\mathrm{SO}_{3}$ groups, Communications in Mathematical Physics 170 (1995), no. 1, 1-20.

[46] Timothy Rainone and Christopher Schafhauser, Crossed products of nuclear $C^{*}$-algebras by free groups and their traces, arXiv:1601.06090 (2017).

[47] Thomas Timmermann, An invitation to quantum groups and duality, EMS Textbooks in Mathematics, European Mathematical Society, 2008.

[48] Stefaan Vaes, Locally compact quantum groups, Ph.D. thesis, Katholieke Universiteit Leuven, 2001.

[49] Alfons Van Daele, Discrete quantum groups, Journal of Algebra 180 (1996), no. 2, 431-444.

[50] Wilhelm Winter and Joachim Zacharias, Completely positive maps of order zero, Münster Journal of Mathematics 2 (2009), 311-324.

[51] _ The nuclear dimension of $C^{*}$-algebras, Advances in Mathematics 224 (2010), no. 2, 461-498. 
Eusebio Gardella, Westfälische Wilhelms-Universität Münster, Fachbereich Mathematik, Einsteinstrasse 62,48149 Münster, Germany

E-mail address: gardella@uni-muenster.de

$U R L:$ https://wwwmath.uni-muenster.de/u/gardella/

Mehrdad Kalantar, Department of Mathematics, University of Houston, Philip Guthrie Hoffman Hall, 3551 Cullen Blvd., Houston, TX 77204, USA

E-mail address: kalantar@math.uh.edu

$U R L:$ https://www.math.uh.edu/ kalantar/

Martino Lupini, Mathematics Department, California Institute of Technology, 1200 E. California Blvd, MC 253-37, Pasadena, CA 91125

E-mail address: lupini@caltech.edu

URL: http://www.lupini.org/ 